\section{AEC}

RESEARCH

\section{and}

DEVELOPMENT

\section{REPORT}

BNWL - 31

$$
\text { C. } 24-
$$

\title{
FLOW VISUALIZATION:
}

A COMPARISON OF STREAMING BIREFRINGENCE

\section{WITH OTHER TECHNIQUES}

MARCH 1965

\section{Extera}

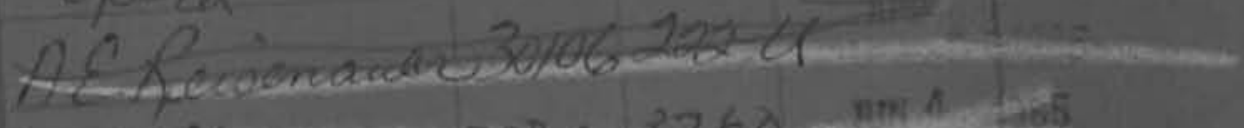

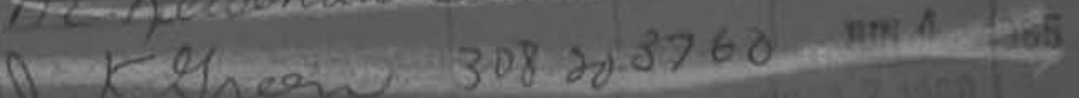

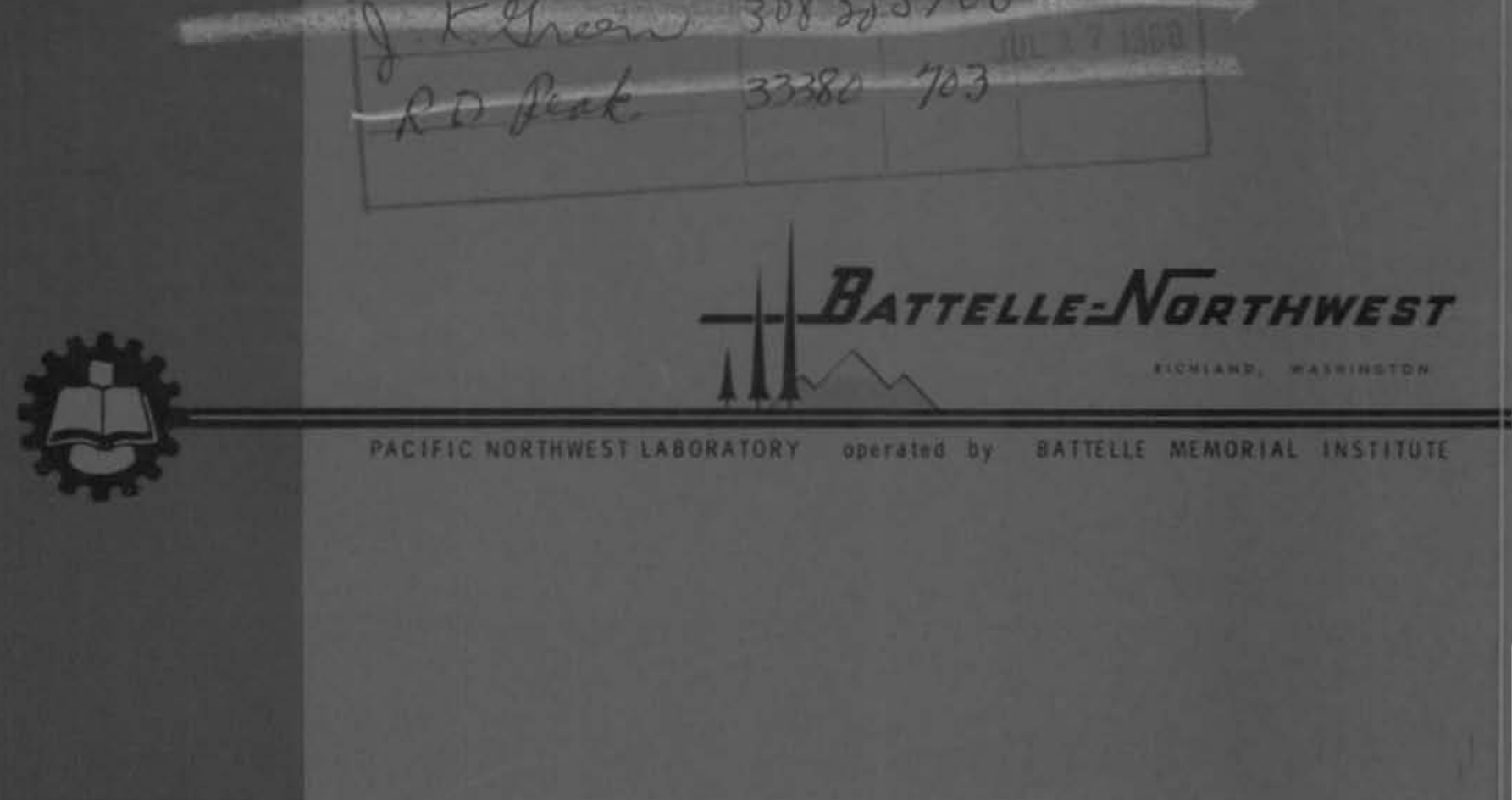




\section{LEGAL NOTICE}

This report was prepared as an account of Gavernment sponsored work. Neither the United States, nor the Commistion, nor any person acting on behalf of the Commissioni

A. Makes any wartenty or representation, expressed oc implied, with respect to the accurasy, com. pleteness, or isefulness of the informotion contained in this report, or that the use af any information. apporalus, method, or process disclased in this report may nol infringe privotely owned rights; or

8. Assumes any llobilities with respect to the use of, or for domages resulting fram the use of any information, opparatus, method, of procest disclosed in this report.

As used in the above, "person octing on behalf of the Commistion" includes ony employete er contractor of the Commission, or employee of such contractor, to the extent that such employee ar cont tractor of the Commission, or employee of such contractor prepares, disueminales, or provides access to, any information pursuant to his employment of contract with the Commisiots or his employment with such contractor: 
BNWL- 31

UC-38, Engineering

and Equipment

(TID-4500, 37th Ed.)

\title{
FLOW VISUALIZATION: \\ A COMPARISON OF STREAMING BIREFRINGENCE \\ WITH OTHER TECHNIQUES
}

\author{
By \\ D. H. White \\ Reactor and Materials Technology \\ March 1965 \\ BATTELLE-NORTHWEST \\ RICHLAND, WASHINGTON
}

Work performed under Contract No. AT(45-1)-1350 between the the Atomic Energy Commission and General Electric Company and under Contract No. AT(45-1)-1830 between the Atomic Energy Commission and Battelle Memorial Institute

Printed by/for the U.S. Atomic Energy Commission

Printed in USA. Price \$3.00. Available from the Clearinghouse for Federal Scientific and Technical Information National Bureau of Standards U. S. Department of Commerce Springfield, Virginia 
TAB LE OF CONTENTS

INTRODUCTION

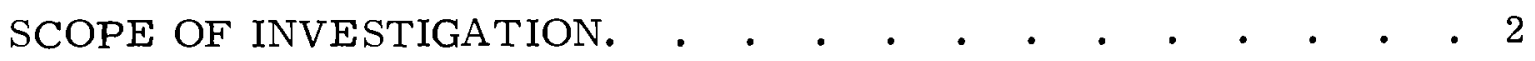

EXPERIMENTAL EQUIPMENT, PROCEDURES AND TECHNIQUES . 3

Preparation and Handling . . . . . . . . . . 5

ELEMENTS OF FLUID VISUALIZATION . . • • • • • • . 6

COMPARISON OF METHODS . . . . . . . . . . . . 8

Flow Separation . . . . . . . . . . . 8

Vena Contracta . . . . . . . . . . . . . 16

Eddies and Wakes . . . . . . . . . . . . 16

General Observations . . . . . . . . • . • 22

CONCLUSIONS.

Limitations and Remarks . . . . . . . . . . 25

APPENDIX A - FLOW VISUALIZATION • . • • • • • . . 27

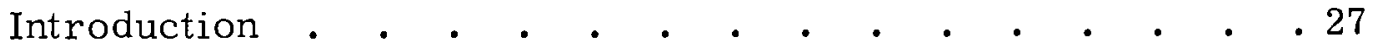

Importance of Visualization Methods . . . . . . . .27

Expected Results. . . . . . . . . . . . . . 28

Methods Available . . . . . . . . . . . . .29

APPENDIX B - ESSENTIAL OPTICS • • • • • • • • . . 31

Properties of Light . . . . . . . . . . . . . 31

Properties of Transparent Materials . . . . . . . 33

Interaction of Light Waves . . . . . . . . . . 35

Polariscope Action . . . . . . . . . . . . .37

Photoelasticity . . . . . . . . . . . . . 39

Streaming Birefringence . . . . . . . . . . . 39

APPENDIX C - PHOTOGRAPHIC EQUIPMENT AND METHODS • . 40

Single Photographs . . . . . . . . . . . . .40

Sequence Photographs . . . . . . . . . . . . 41

High Speed Movies . . . . . . . . . . . . 41 
APPENDIX D - DESCRIPTION OF EQUIPMENT • • • • • • . 42

Flow Channel and Models . . . . • . • • . . 43

Recirculating Loop Equipment . . . • • • • . . . 44

Supercentrifuge • • • • • • • • • • . • . . 44

Viscometer . . . . . . . . . . • . . . . 44

APPENDIX E - BACKGROUND, THEORY, AND PROPERTIES

OF SBR SOLUTIONS • • • • • • • • • • • • • • • 47

Background of Use • . • . • . • . • . . • . 47

Theory of Streaming Birefringence . . . . . . . . 49

Properties of Bentonite Sols . . . . . . . . 51

Flocculation . . . . . . . . . . . . . . 52

Gelation . • • • • . • • • • • • • . 52

Peptization . . . . . . . . • • • . . . 53

Anomalous Viscosity . . . . . . . . . . 53

APPENDIX F - PREPARATION AND HANDLING

OF BENTONITE SOLUTIONS • • • • • • • • • • • 55

Preparation . . . . . . . . . . . . . 55

Handling . . . . . . . . . . . . . . 56

APPENDIX G - PREPARATION AND HANDLING

OF OTHER VISUALIZATION TECHNIQUES • • • • • • • 57

Tracers • • • • • . • . . • • • • • . . 57

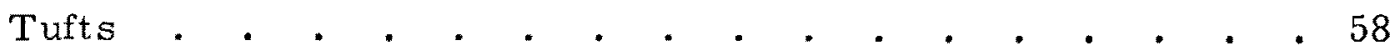

Dye . . . . . . . . . . . . . . . . 58

APPENDLX H - ANALYSIS TECHNIQUES

OF LAMINAR FLOWS UTILIZING SBR PROPERTIES • • • $\quad 60$

Prados Analysis for Two-Dimensional Laminar Flow . . 61

Examples of Specific Cases . • • • • • • • 67

Limitations . . . . . . . . . . . . . 68

Summary of Kemp's Investigation • • • • • • • • 69

Summary of Wayland's Theoretical Analysis. . . . . . 70

Remarks . . . . . . . . . . . . . . . . 71

REFERENCES • • • • • • • • • • • • • • • • . 73 


\section{LIST OF FIGURES}

Figure I

Figure II

Figure III

Figure IV

Figure V

Figure VI

Figure VII

Figure VIII

Figure IX

Figure X

Figure XI

Figure XII

Figure XIII

Figure XIV

Figure XV

Figure XVI

Figure XVII

Figure XVIII

Figure XIX

Figure XX

Figure XXI

Figure XXII

Figure XXIII

Figure XXIV

Figure XXV
Models and Channel .

Recirculating Flow Facility.

Four Visualization Methods -

Around a Cylinder . . . . . . . . . 10

Cylinder Flows - Dye and SBR . • • • • • • 11

Table of Measured Angle of Separation . • • . . 12

Four Visualization Methods -

Separation at Throat of Restrictor . . . . . 13

Three Visualization Methods -

Separation in a Diffuser. . . . . . . . . 15

Four Visualization Methods -

Vena Contracta for an Orifice . . . . . . . 17

Tracings of Vena Contracta . • . • • • • . . 18

Results of Measured Apparent Thickness. • • . 19

Eddies Associated with Orifice. • • • • • . 20

Cylinder Wakes . • . . . • • • • • . • . 21

Laminar and Turbulent Flows - SBR . . . . . . 23

Flow of Tracers and SBR . • • • • • • • . 24

Reflection and Refraction at a Boundary . . . . . 32

Crystal Transmittance of Light. • • • • • • . 34

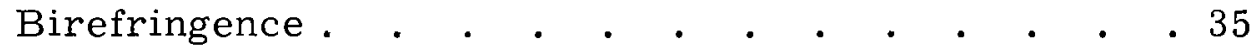

Wave Interaction . . . . . . . . . . . . 36

Polariscope Operation . • • • • • • • . . 38

Schematic of Flow - Flow Facility. • • • • . . 42

Supercentrifuge . • • . • • • • • • • . 45

Viscometer and Spindles. • . . • • . • . . 46

Apparent Viscosity of Hectorite Solutions. . . . . 54

Elemental Stress Relationships. • . . . . . . 61

Transformation Relationships . . . . . . . . 63 


\section{ACKNOWLEDGMENTS}

This study was possible only because of the help and encouragement of numerous Battelle-Northwest personnel at Hanford, especially D. P. Schively, my supervisor; Dr. J. M. Batch, my Battelle-Northwest advisor; Dr. F. R. Zaloudek; Dr. W. J. Love, now with Advanced Technology Laboratories, General Electric; and L. P. Reinig, of N-Reactor Department, General Electric, who assisted with the sponsorship.

The invaluable assistance and recommendations, despite major inconveniences, of Dr. M. E. Childs, Chairman of my University Advisory Committee, are gratefully acknowledged and deeply appreciated.

The work was performed under Contract No. AT(45-1)-1350 by and between the Atomic Energy Commission and General Electric Company at the Hanford Laboratories, Hanford Atomic Products Operation, Richland, Washington and under Contract No. AT(45-1)-1830 by and between the Atomic Energy Commission and Battelle Memorial Institute. 


\begin{abstract}
The flow visualization technique known as streaming birefringence (SBR) was studied by direct comparison with three other techniques; small spherical plastic tracers, cotton thread surface tufts, and dye injection.

Comparative tests on four differently shaped, two-dimensional models were run. Reynolds numbers for the incompressible fluids varied from about 1000 to about 6000. These tests showed that an SBR solution of bentonite clay compared favorably with other visualization techniques for the (a) flow separation around a cylinder, (b) flow separation at the throat of a restrictor, (c) vena contracta of an orifice, and (d) transverse flow in a rectangular channel. The SBR fluid compares unfavorably or questionably for diffuser separation and circular eddies.

It is concluded that the SBR flow visualization technique has potential for use as a visualization tool, requires skill and care in use, and should be investigated in greater depth.
\end{abstract}




\section{FLOW VISUALIZATION: \\ A COMPARISON OF STREAMING BIREFRINGENCE WITH OTHER TECHNIQUES}

\section{INTRODUCTION}

During the development period of the New Production Reactor (NPR) at Hanford, Washington, a hydraulic device, called a flow restrictor, proposed for use in the reactor was tested and found to be only one-third as efficient as expected. Large inefficiencies cannot be tolerated in the reactor primary coolant system. With the hope of lowering the pressure losses, an investigation of the flow patterns around this restrictor was planned. A technique which would allow visualization of the flow was required for this study, and the method utilizing the streaming birefringence of a bentonite clay solution was considered as a possible means of making the investigation.

However, after a literature search was made and preliminary tests were run, certain basic questions about the general effectiveness of the bentonite clay solution remained unresolved. In short, the problem was that the restrictor could not be studied using the visualization tool of streaming birefringence until some of the basic, fundamental properties of this evaluation tool itself were better understood. This thesis presents the results of a study designed to define more adequately some of these properties.

The technique is a form of flow visualization and the ability to make fluid flow phenomena visible to the human eye has played an important and integral part in furthering the understanding of fluid mechanics, both as a science and as an engineering tool. Therefore, a contribution to the understanding of fluid visualization has broad potential applications. Appendix A gives a brief résumé of the philosophy and background of flow visualization. 


\section{SCOPE OF THE INVESTIGATION}

The object of this investigation was to determine whether or not the technique based on the properties of streaming birefringence is a good flow visualization tool for turbulent flow conditions. Although some flow cases in the laminar range were investigated, major emphasis was on flows in the turbulent range.

The tests herein repoxted indicate that this technique accurately reflects a number of the important characteristics of the flow and, therefore, has potential as a visualization tool. Its use, however, requires care and understanding as there is much to learn about this method. Further investigation of this technique is desirable.

The visualization obtained from this method is the result of certain optical properties. Many substances are considered to be optically isotropic, which simply means that the velocity of light passing through them is independent of the direction of the light vector. The properties of certain substances to temporarily become anisotropic when influenced by external force fields is known as double refraction or birefringence. For electrical forces, this is known as the Kerr effect; for mechanical stress, it is known as photoelasticity, and for shearing stresses associated with flowing fluids this property is known as flow double refraction, streaming double refraction, flow birefringence, photoviscous effect, or streaming birefringence (SBR). The first name is probably more descriptive to most people, but streaming birefringence is used by the majority of investigators and will be used throughout this report. A short review of the essential optics required to understand the principle of birefringence is included as Appendix $B$.

The tests were arranged in this investigation in an attempt to compare the use of the SBR of a bentonite solution directly with other visualization techniques at dyramically similar flow conditions. Techniques utilizing small plastic tracers, surface tufts, and dye injection were employed. This investigation was further limited to twodimensional models and incompressible fluids with Reynolds numbers ranging from just below 1000 to just about 6000 . 
The comparison of the four techniques was made using photographs and, where applicable, tracings of these photographs. The photographic equipment used and an outline of the photographic procedures followed is given in Appendix $\mathrm{C}$.

Available funding restricted the investigation to the use of equipment on hand.

EXPERIMENTAL EQUIPMENT, PROCEDURES, AND TECHNIQUES

The test equipment consisted of a transparent flow channel, a recirculating flow facility with pump, valve, flow meter, tank, and piping, required optical and photographic equipment, and four flow models.

The models were (a) a cylinder, (b) an orifice, (c) a venturi, and (d) an NPR restrictor. Each was mounted to a piece of lucite, which was sized to fit an opening in the wall of the six-foot long flow channel. The inside channel dimensions were $2 \times 2$ inches. Figure $\mathrm{I}$ is a photograph of the models and the channel.

The recirculating flow facility was simply a 200 liter stainless steel tank connected through a small centrifugal pump, a precision rotometer, and a throttling valve to the flow channel. Fluid from the channel was discharged back into the tank to complete the cycle. A photograph of the facility is shown in Figure II while detailed information on the equipment can be found in Appendix D.

The test procedures used in this investigation are based on direct comparison of different flow conditions. This direct comparison has meaning only when the systems are dynamically similar. For this case, where frictional and inertial forces predominate, equality of Reynolds numbers is a necessary and sufficient condition for dynamic similitude. $(1)^{*}$ Comparisons between the various systems were made only after the Reynolds numbers for that flow condition had been made equal.

Numbers in parentheses refer to references at the end of the report. 


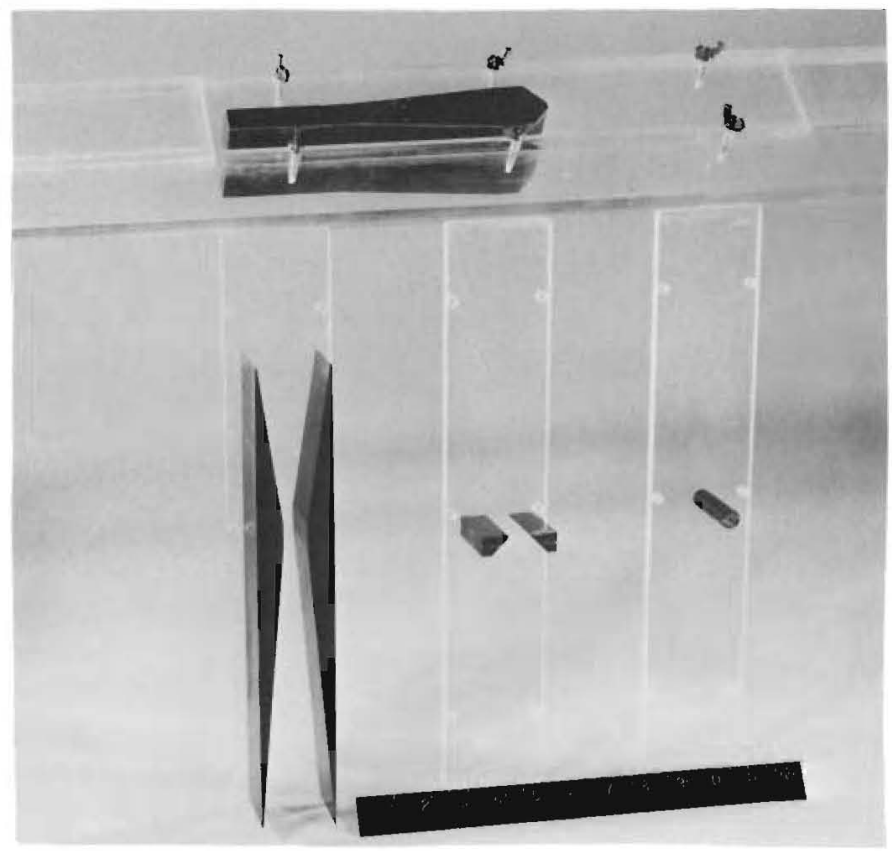

\section{FIGURE I}

Models and Channel

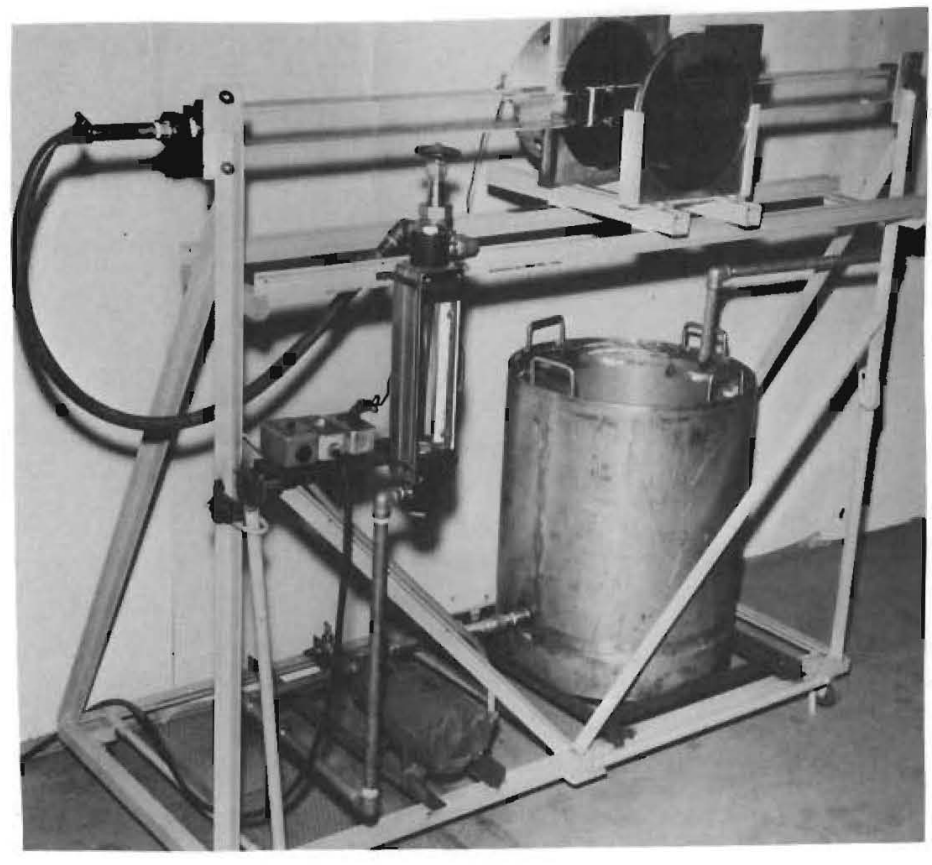

FIGURE II

Recirculating Flow Facility 
First, tests were run using the SBR fluid. Next, tests using the glycerol-water mixture and polystyrene spheres, followed by the cotton thread surface tufts tests, and the potassium permanganate dye tests, in that order. The last three techniques, tracers, tuft deflection, and dye streaks, are common flow visualization techniques and are believed to be reasonable methods with which to evaluate some of the characteristics of the less familiar SBR technique.

Preparation and Handling. The techniques of preparing and handling the various visualization tools strongly influence the test reliability and results. This is particularly true of bentonite solutions. Bentonite is a naturally occurring clay which forms a collodial suspension when mixed with water. This colloid is a non-Newtonian fluid which will gel if not prepared and handled properly and which exhibits anomalous viscosities. The anomalous viscosities could be a great drawback in fluid analysis work. Measurements by Kemp (2), however, show this effect to be rather small. Viscosity measurements made during these tests on a modified viscometer showed surprisingly consistent values for viscosity. Appendix E - Background, Theory, and Properties of SBR Solutions - gives a short summary of SBR history and includes results of Kemps viscosity measurements.

The preparation of a useable solution is tedious and somewhat of an art, requiring the proper clay and proper equipment. Some of the problems involved are pointed out in Appendix F.

Polystyrene spheres were chosen as tracer particles. Allen ${ }^{(3,4,5)}$, Winter and Deterding ${ }^{(6)}$, and Van Meel and Vermig ${ }^{(7)}$, have all used plastic tracers and describe equipment and techniques considerably more sophisticated that those used in these tests. Sawdust particles as tracers were tried and gave a fairly good visual pattern, but they could not be effectively photographed.

Before acceptable photographs were obtained, the fluid density had to be adjusted to match the density of the plastic. Glycerol was added to the water to achieve this neutral density match. 
To prepare the tufts for testing, small depressions were made in the model surface with a pointed scribe. Tufts of thread were inserted at right angles and fixed with a drop of plastic solvent.

The dye used in the dye tests was an aqueous potassium permanganate solution. It was introduced into the stream with a hypodermic needle bent to allow insertion of dye parallel to the mean fluid velocity.

Details on the preparation and use of the tracers, tufts, and dye can be found in Appendix G.

The handling, preparation, limitations, and techniques of use associated with any visualization method are strong factors in its effectiveness as a fluid mechanics tool and, thus, must be carefully considered by any investigator attempting to choose the optimum method for his particular study. ELEMENTS OF FLUID VISUALIZATION

Before different visualization methods are compared, it is desirable to quickly review the meaning of certain terms commonly used in flow visualization work.

A number of basic visualization concepts associated with fluid flow include path lines, streak lines, streamlines, and isochromatic lines. The relationships between them must be clearly understood if confusion is to be avoided.

A path line is a line made by a single, elemental fluid particle over a period of time. ${ }^{(8)}$ An example is a time exposure photograph of a plastic sphere in a flowing fluid.

A streak line is a locus line in a field of flow, at any given instant of time, which connects the temporary location of all particles that have passed through a given upstream contamination point. (9) An example is a flash photograph of a dye streak in a flowing channel.

A streamline is a purely mathematical concept defined as a line which is everywhere tangent to the velocity vectors. 
These three lines coincide only when there is steady flow. Steady flow is defined as flow in which velocity, pressure, density, and other such characteristics at a point do not change with time. ${ }^{(8)}$ It should be noted that steady and unsteady flow can exist at two points near each other in the same flow. For example, Birkhoff and Zaratonello ${ }^{(10)}$ note that wakes at Reynolds numbers over 1000 tend to be turbulent. The random fluctuating velocity changes in such a wake causes point flow to be unsteady while a short distance away, in the free stream, point flow is steady.

Visualization of turbulent flow is dependent on the frame of reference. (11) The exact mechanism of turbulence is not completely established at present, but it is believed that macroscopic fluid "lumps" agglomerate, fluctuate, translate, and disintegrate in a random manner and at high frequency. It is common to express the $u$ component of fluid velocity as

$$
u=\bar{u}+u^{\prime}
$$

where $\bar{u}$ is the the time-average portion and $u^{\prime}$ is the velocity fluctuation portion of $u$. When $\bar{u}$ is taken over a sufficiently long period of time to be time independent, $\bar{u} !$ is, by definition, equal to zero. This concept is stated or implied in many references $(8,9,10$, and 12 to name a few) and is restated here because it helps explain the nature of visualization of turbulent fluid flows. 'Thus, for turbulent flows with time taken long enough so that $\bar{u}^{\prime}$ equals zero, path lines can be short lines (similar to laminar path lines) while streak lines tend to become mixed and extend over the whole conduit area. Other techniques, such as shadowgraph pictures, which are not dependent on an average $u^{\gamma}$, result in pictures of rapidly changing, small, closely spaced lines.

The fourth basic concept mentioned above is usually associated with photoelasticity. An isochromatic line is the locus of points of equal relative retardation of light and are therefore positions at which the principle mean stress has a constant value. (13) Each individual 
isochromatic line is a region of light interference, a line of constant color when white light is used, and is made visible using circularly polarized light. (14) An isochromatic line integrates fluid phenomena across the entire channel width.

Flow visualization is most useful when a well verified association exists between the flow regime and its governing parameters. Governing parameters include such things as shape fluid properties, and time. The association of particular flow regimes with these parameters makes possible the non-mathematical but descriptive analysis which is sometimes referred to as "semi-quantitative." Wherever possible, this technique was used to evaluate these tests.

When applied to laminar flows, SBR will yield quantitative data in a manner somewhat analogous to photoelastic analysis. Such quantitative analysis for flowing fluids has been attempted by Dewey ${ }^{(15)}$, Prados ${ }^{(14)}$, Kemp ${ }^{(2)}$, and Wayland and Sutera. (16) The analysis technique of Prados is outlined in Appendix $\mathrm{H}$ and short summaries of relationships used by Kemp and Wayland are given. COMPARISON OF METHODS

Specific areas of comparison were chosen to make the evaluation more meaningful and systematic. For maximum value, areas were picked that would normally concern the engineer in real problems.

These areas were (a) points of boundary layer separation, (b) vena contracta thickness, (c) patterns of eddies and wakes, and (d) other general observations of the flow. Comparisons were made only between the flows of equal Reynolds number and therefore dynamically similar situations.

Flow Separation. The first area chosen for direct comparision was the phenomenon of boundary layer separation for the cylinder, restrictor, and diffuser. 
Figure III, gives a comparison of flows around a right circular cylinder. All flows shown in Figure III have a Reynolds number of 4690 . (All Reynolds numbers were mean channel values calculated using hydraulic radius and mean channel velocity.)

The angle of separation, $\varphi$, is defined as the angle from the front stagnation point to the point of apparent separation. Note in Figure III that for the dye and SBR techniques it is greater than $90^{\circ}$, and that separation is not defined with the tufts or tracers.

Figure IV shows the dye and SBR photographs in greater detail. The Reynolds number for all flows is 3140 . Note the different dye patterns resulting from different elapsed times between dye injection and shutter opening.

Tracings were made of a number of SBR and dye photographs and $\varphi$ was measured as accurately as possible. Figure $V$ is a tabulation of these measurements. Attempts to use this same comparison technique on tracer photographs were unsuccessful because the tracer particles were too large to be contained within the boundary layer. It is of interest to note that the tracer appeared to begin pulling away from the cylinder about $70^{\circ}$ to $80^{\circ}$ back of the stagnation point.

A comparison of these results with experimental and theoretical results by others is valuable. Knudsen and Katz ${ }^{(17)}$, in summarizing results of Thom, Green, and Fage-Falkner, note that the actual point of separation for cylinders is influenced by the test channel, the history of the main stream before it comes in contact with the sphere, the Reynolds number, and the surface of the cylinder. This tends to verify the visual observation during the SBR and dye tests which showed minor fluctuations and changes in the flow picture at the cylinder surface, especially at the higher Reynolds number. 


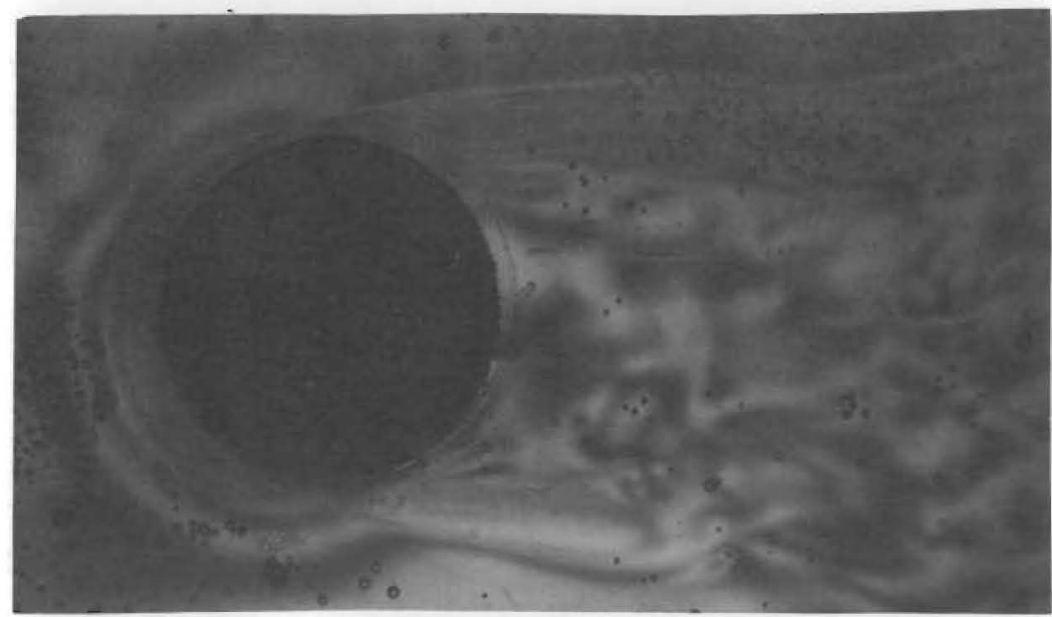

SBR

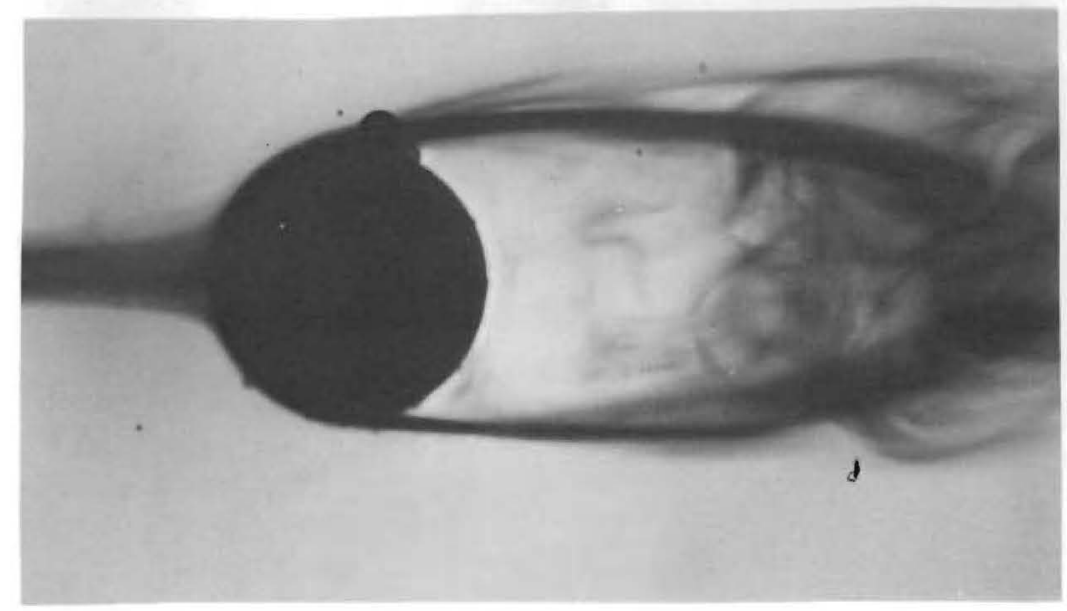

Dye

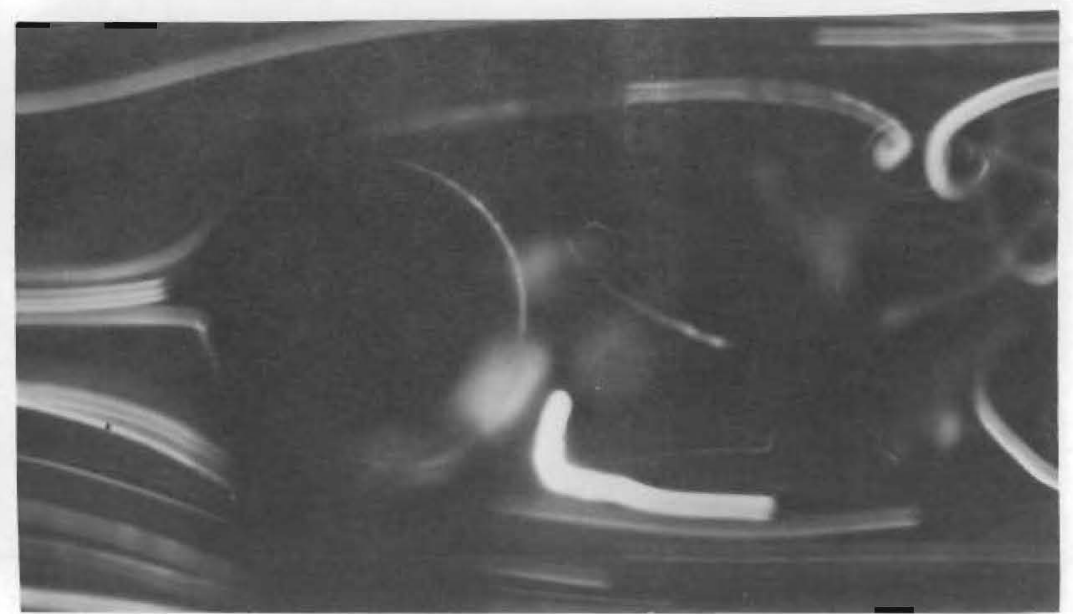

Tracers

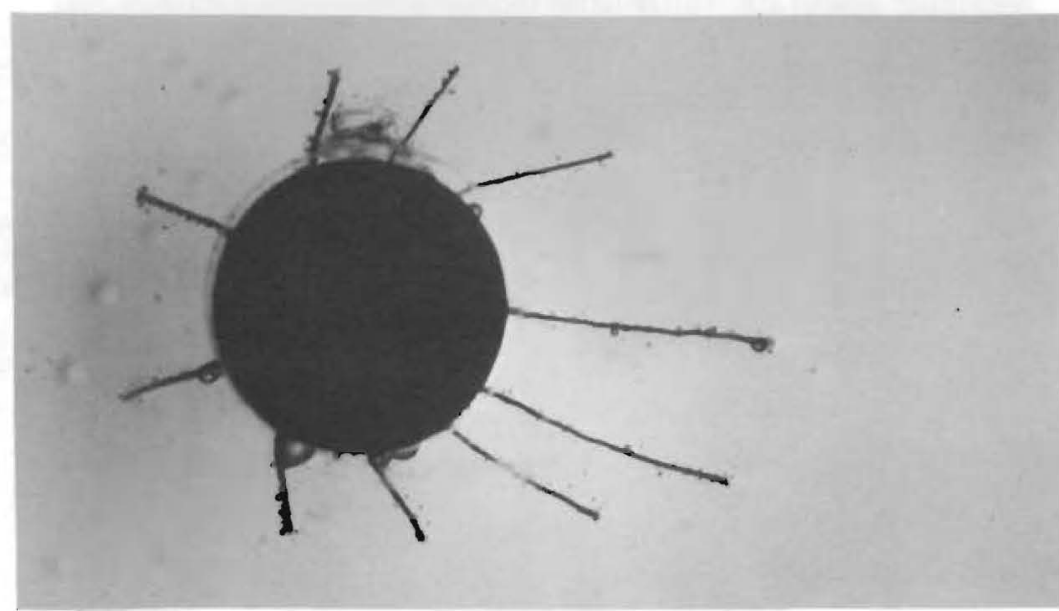

Tufts

\section{$\stackrel{1}{\stackrel{1}{\circ}}$}

Four Visualization Methods - Separation Around a Cylinder 


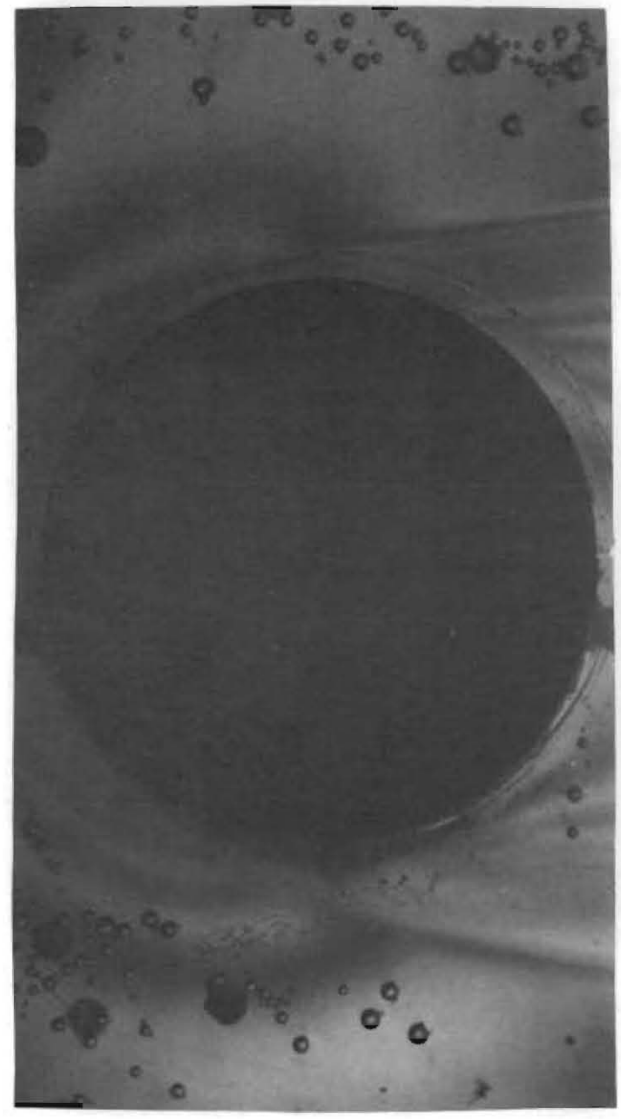

SBR

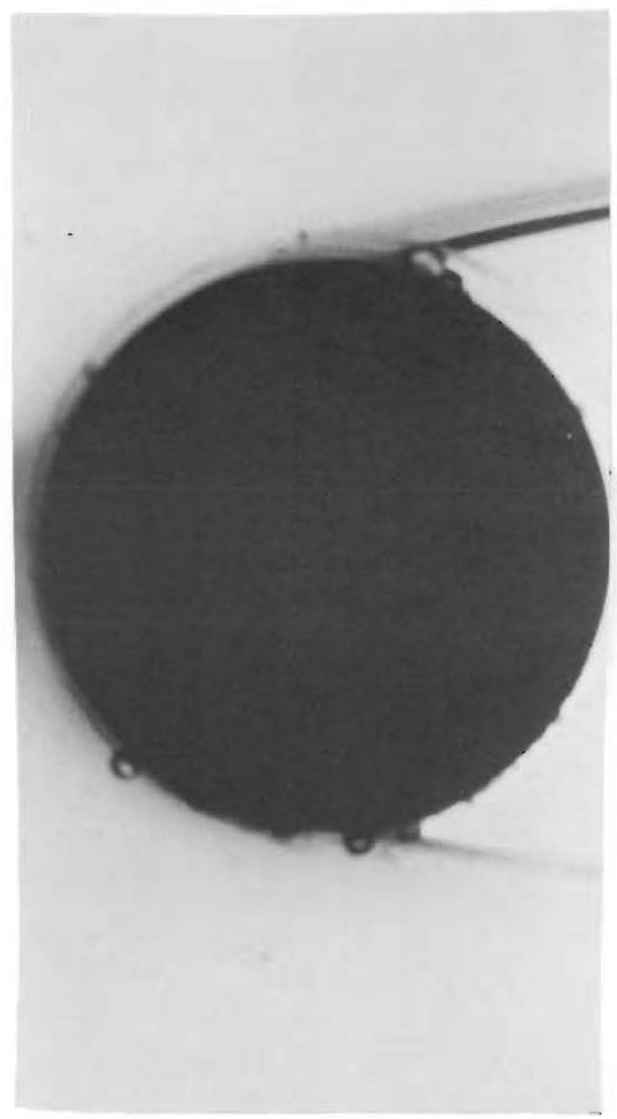

Dye

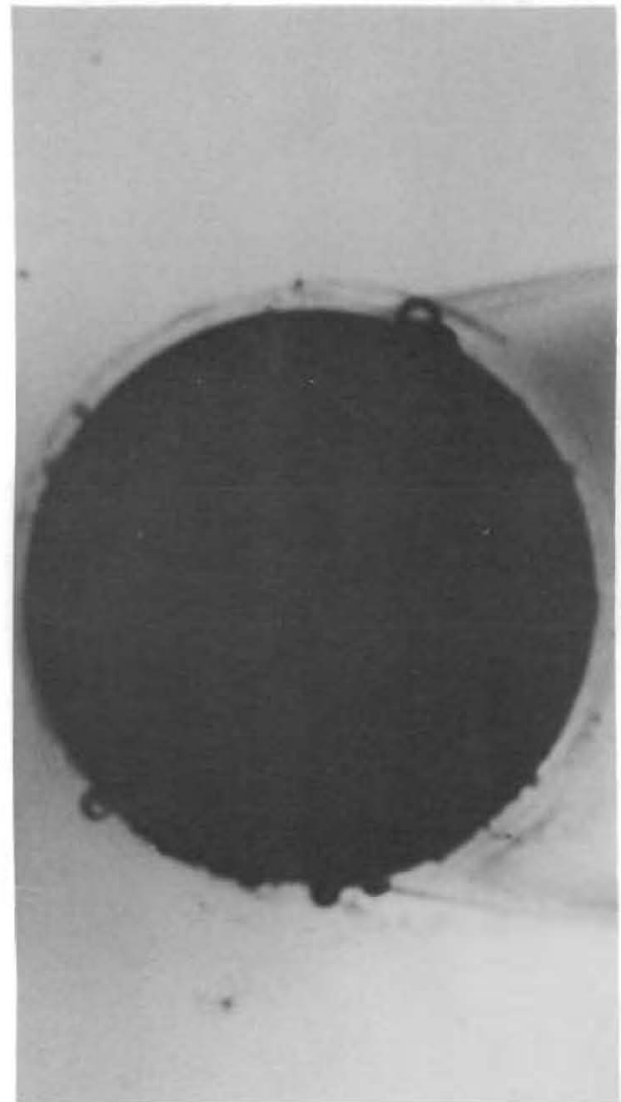

Dye-Shutter Retarded

FIGURE IV 


\begin{tabular}{|c|cc|c|c|}
\hline \multirow{2}{*}{$\begin{array}{c}\text { Reynolds } \\
\text { Number }\end{array}$} & \multicolumn{4}{|c|}{$\begin{array}{c}\text { Angle of Separation, } \varphi, \\
\text { in Degrees }\end{array}$} \\
\cline { 2 - 5 } & \multicolumn{2}{|c|}{ SBR } & \multicolumn{2}{|c|}{ Dye } \\
\hline \hline 1570 & 110 & 96 & 105 & 104 \\
3140 & 117 & 106 & 101 & 96 \\
& 117 & 112 & 110 & 98 \\
& & & 106 & 105 \\
4690 & 111 & 104 & 109 & 107 \\
6260 & 109 & 109 & 98 & 107 \\
\hline
\end{tabular}

\section{FIGURE V}

Table of Angle of Separation, $\varphi$, for SBR and Dye

Schlichting ${ }^{(18)}$ calculates the exact solution, using the Blasius series for $u$, of the steady state boundary layer equations and gets a $\varphi$ of $110^{\circ}$ and then calculates the approximate solution, as given by Pohlhausen, as $109.5^{\circ}$. These equations are within $1 \%$ of measurements as made by Hiemenz. $(12,18) \operatorname{Binder}^{(8)}$ and others $(12,17,19,20)$ indicate that the angle of separation remains fairly constant over a wide range of Reynolds numbers.

Figure $V$ gives values of $\varphi$ measured in these tests from $96^{\circ}$ to $117^{\circ}$. Keeping in mind that separation is influenced by many factors and that these tests were not designed for careful measurements, merely general comparisons, this spread of values for the dye and the SBR gives a general indication of the phenomenon. Tests which isolate or control more parameters than these tests are required to establish complete agreement with previous investigators.

In addition to flow separation on the cylinders, separation of the flow from the surface of the model was noted at the throat of the NPR annular flow restrictor. A direct comparison of this flow regime by the four visualization techniques is given in Figure VI. The Reynolds number for these pictures was 6260. The surface tuft pictures are again of no value. 


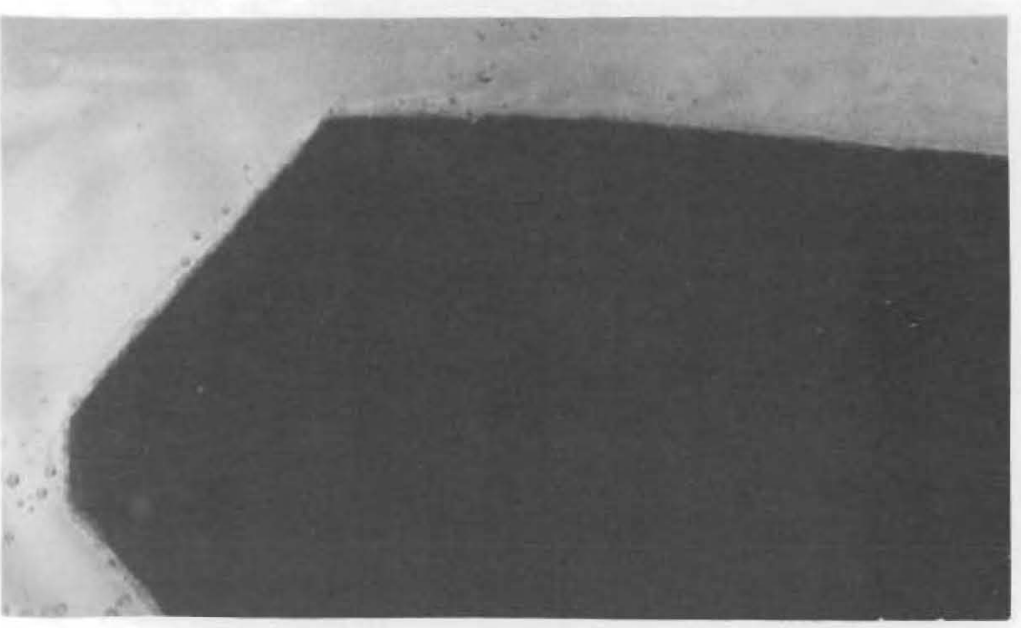

SBR

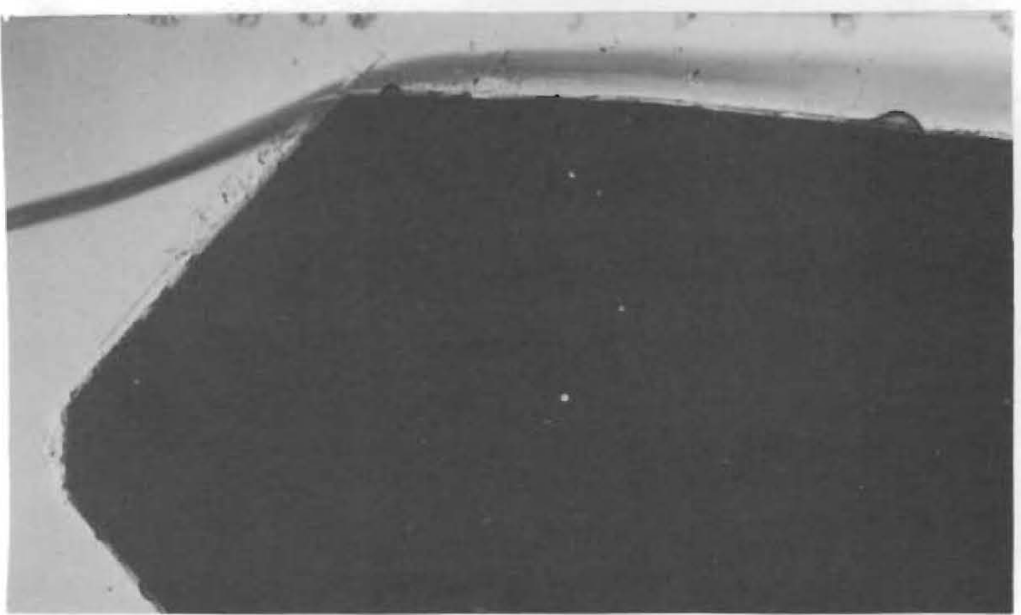

Dye

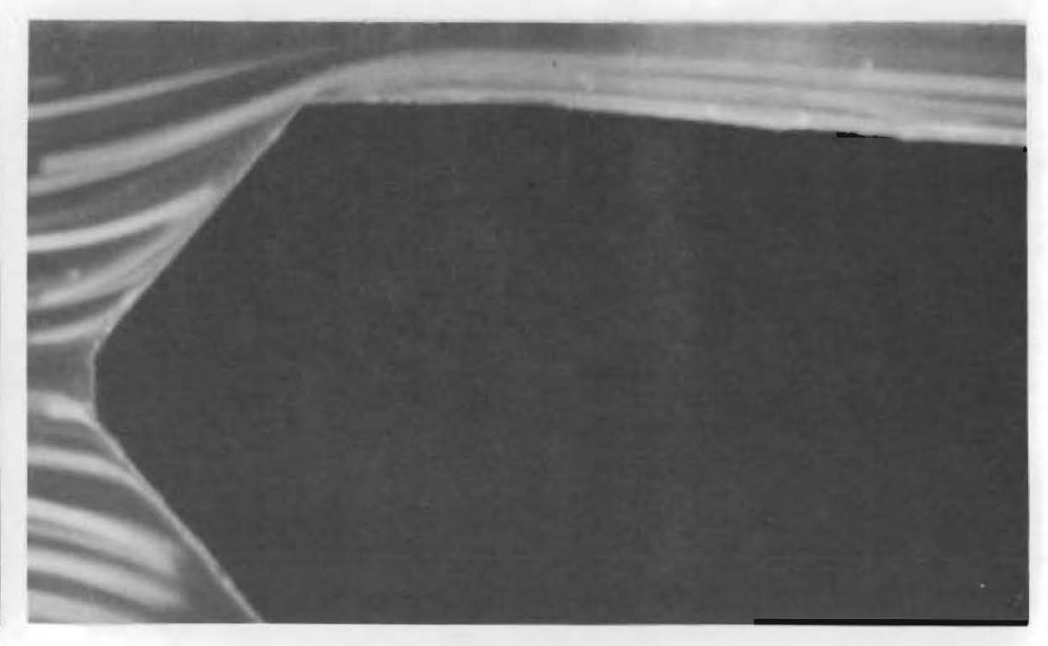

Tracers

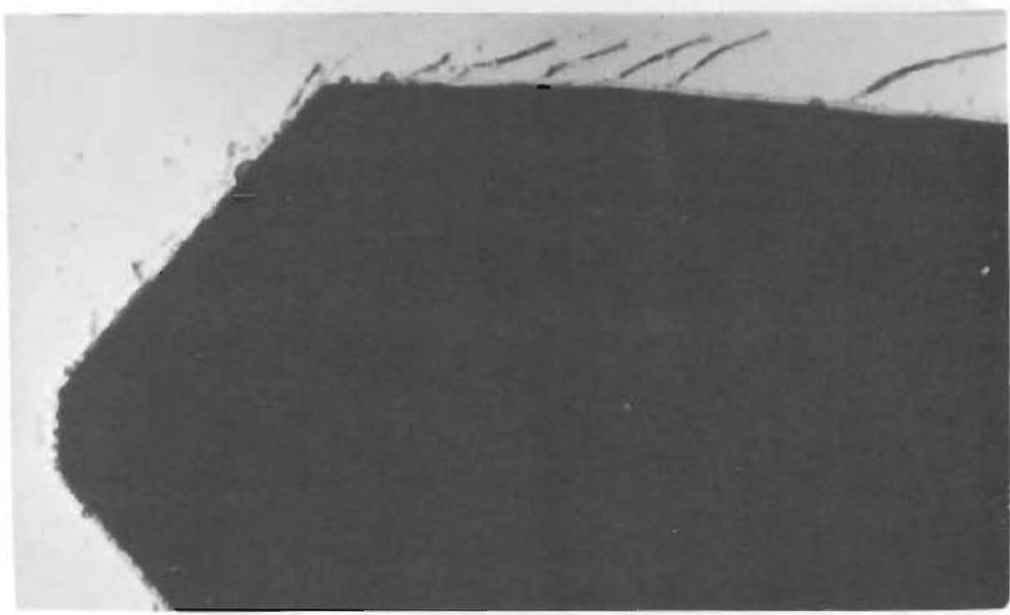

Tufts

FIGURE VI

Four Visualization Methods - Separation at Throat of Restrictor 
It is felt that separation is caused by the steep inlet shape. Notice that the flow appears to "overshoot" the forward edge of the throat, reattaching itself further downstream. This curved path of the flow from the front edge of the throat to the point of reattachment reduced the effective throat height. Measurements of tracings near the point of major restriction shows a reduction in effective throat height of $14.6 \%$ using the SBR technique, $14.3 \%$ using the tracer technique, and between 19 to $20 \%$ using the dye technique.

A more thorough investigation of this particular effect would undoubtedly yield more valuable and accurate results. The fact to be emphasized here is that the SBR technique defines the same separation effect as does the tracers and dye and that measurements compare quite closely.

A third area where boundary layer separation can take place is in a sufficiently adverse (positive) pressure gradient, such as is found in diffusers. Figure VII shows the pattern in the diffuser section of a Herschel venturi. The Reynolds number is 6260. Tracer photographs were not possible because the model blocked the vertical light used with tracer photography.

Tracings were made of these pictures. Measurements were taken between an arbitrary reference point near the rear of the throat ard the apparent point of separation. This length was 0.62 inch for the dye picture and 1.02 and 1.12 inches for the SBR picture.

The variation between these measurements is rather large and in the absence of more detailed understanding of the fluid conditions at this point, these results must be considered as inconclusive. A number of explanations could be advanced explaining this difference in results, such as: (1) the point chosen as "apparent separation" is really the point of turbulent inception in an already separated boundary layer, or (2) an inadvertent pulsation occurred during the dye injection. However, "it appears" that further, more detailed tests are necessary to explain separation phenomenon in diffusers adequately. 


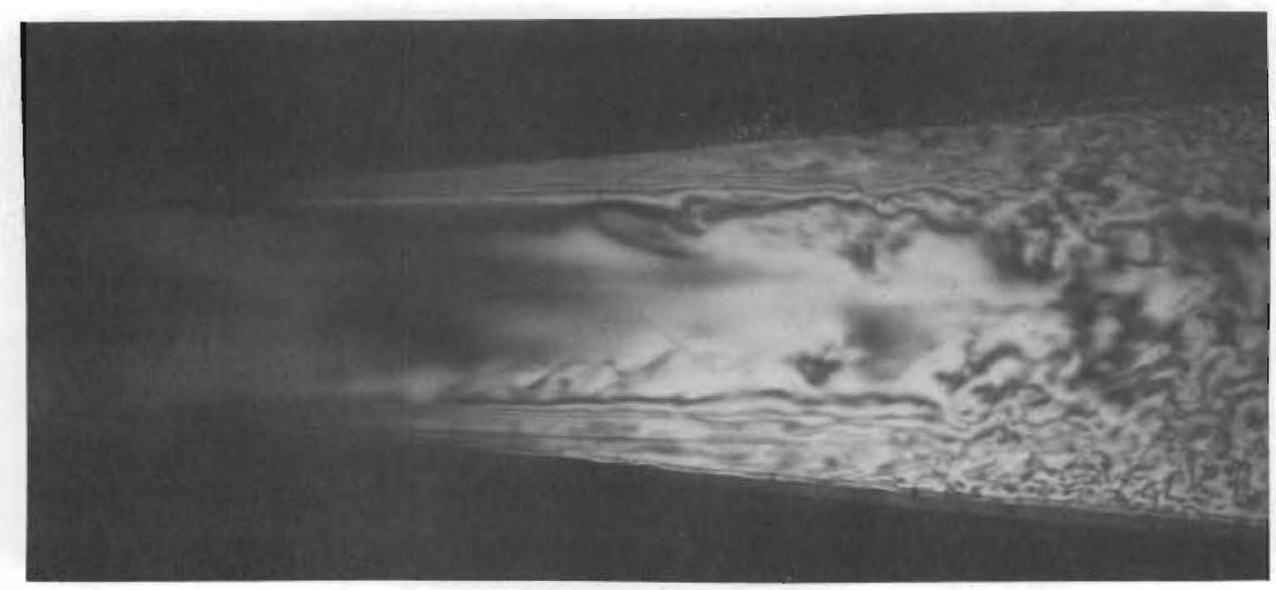

\section{SBR}

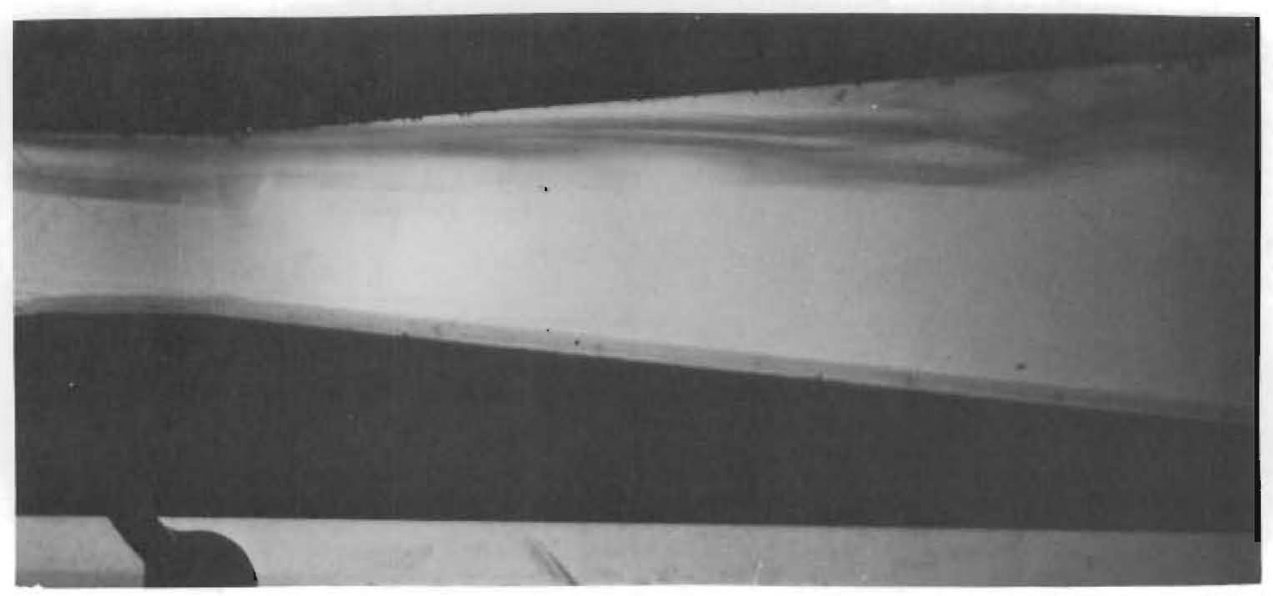

Dye

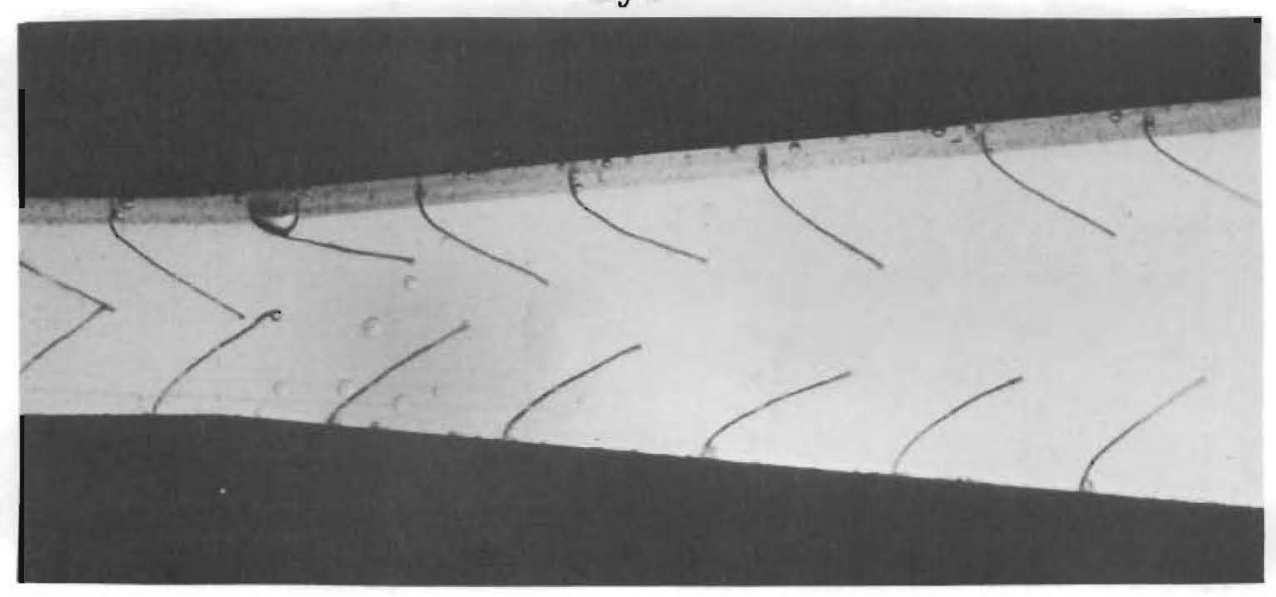

Tufts

FIGURE VII

Three Visualization Methods - Separation in a Diffuser 
It should be noted that close observation of the photographs reveal distinctly different patterns upstream and downstream of the point chosen as "apparent separation." Whether this is separation or not must be based on further investigation.

Vena Contracta. The second area chosen for direct comparison was the nature of the vena contracta associated with the orifice model. Figure VIII shows the vena contracta as visualized by the four different techniques at a Reynolds number of 1880 .

The vena contracta thickness, $T$, is the point of greatest jet contraction. Streeter $(21)$, using the Schwarz-Christoffel theorem for a two-dimensional, sharp-edged orifice, gives a value for $\mathrm{T}$ of 0.611 times the orifice opening. Thus, for our model throat thickness of $5 / 8$ inch, the theoretical $\mathrm{T}$ would be 0.382 inch.

Figure IX shows line drawings made from tracings of the photographs. The tracings in this study tend to be averaging and interpretive processes and result in simplifications of complicated phenomena. These somewhat idealized sketches were then measured as carefully as possible. The results are given in Figure $\mathrm{X}$.

This was the only test where surface tufts could be considered even partially successful, and then only at the higher Reynolds numbers where the fluid velocity was great enough to overcome the thread rigidity.

Figure $\mathrm{X}$ shows an appreciable scatter of values for the average apparent vena contracta. The values do, however, fall near the expected value obtained by calculation. Which values are "correct" and which are in "error" can be answered conclusively only by more detailed and restrictive tests.

Eddies and Wakes. The third area chosen for direct comparison was the behavior of certain small circular eddies associated with flow in the separated region ahead of the orifice model and with those eddies associated with flow in the wake of a cylinder. 


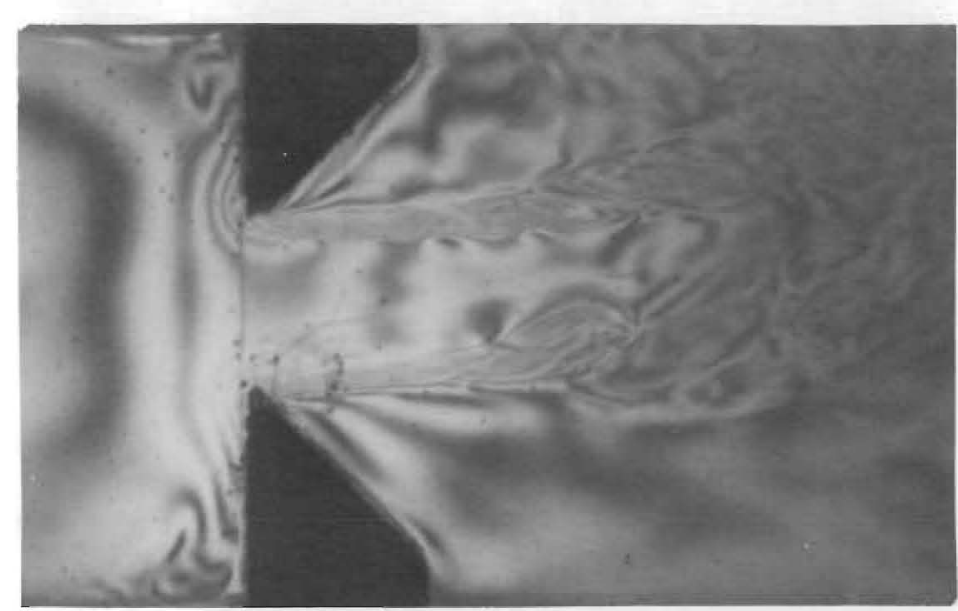

SBR

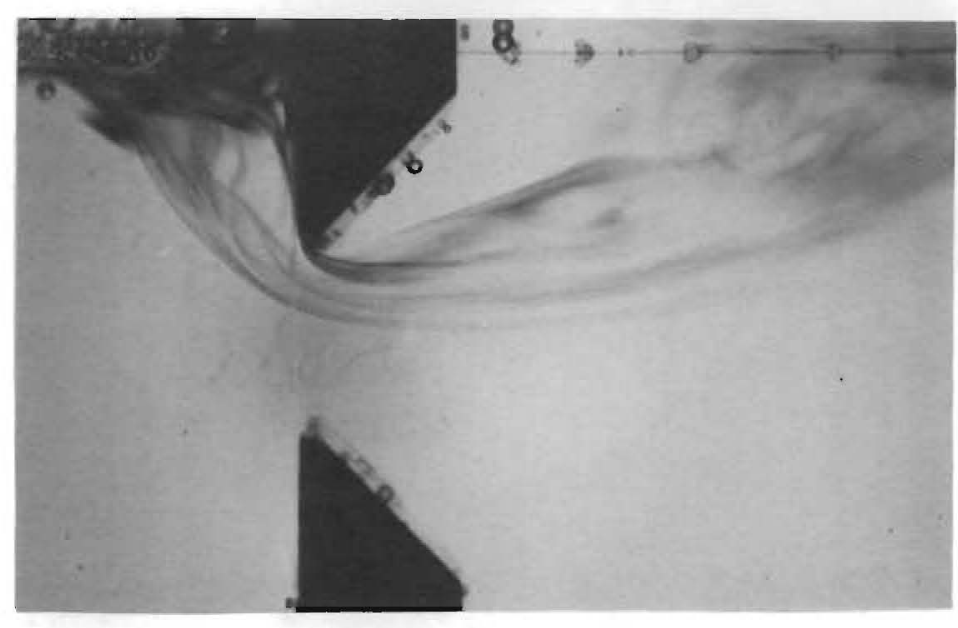

Dye

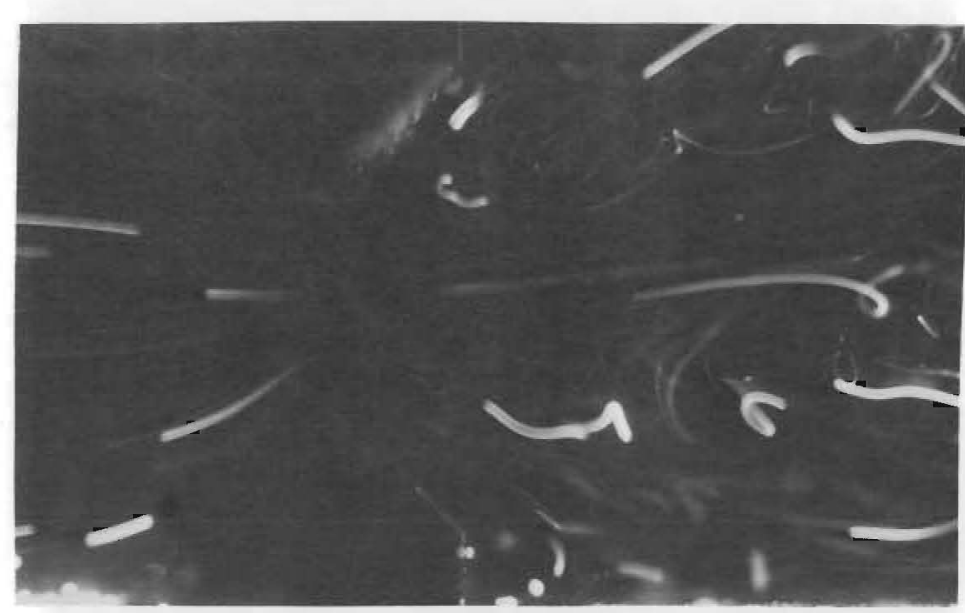

Tracers

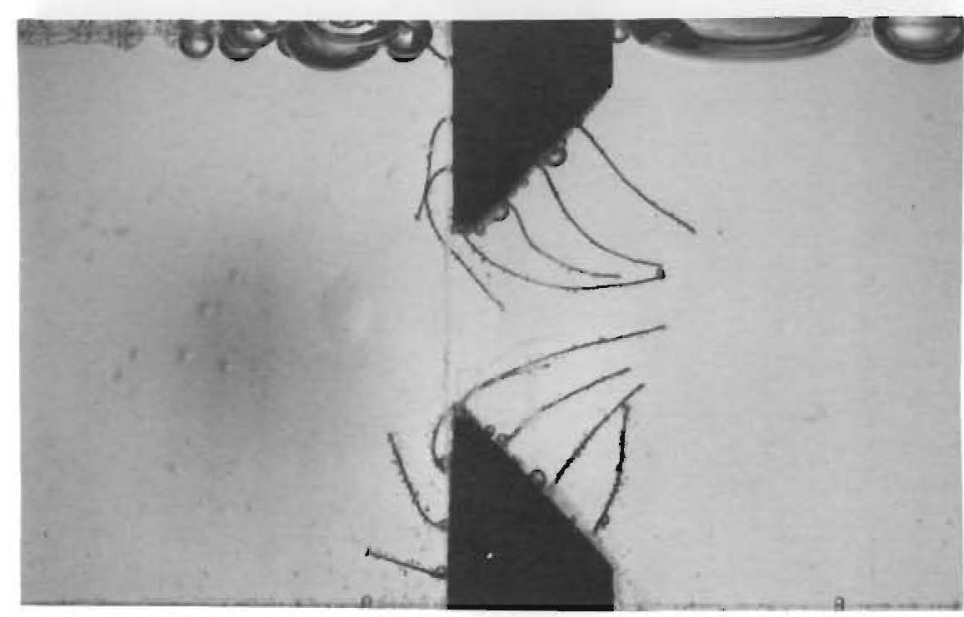

Tufts 

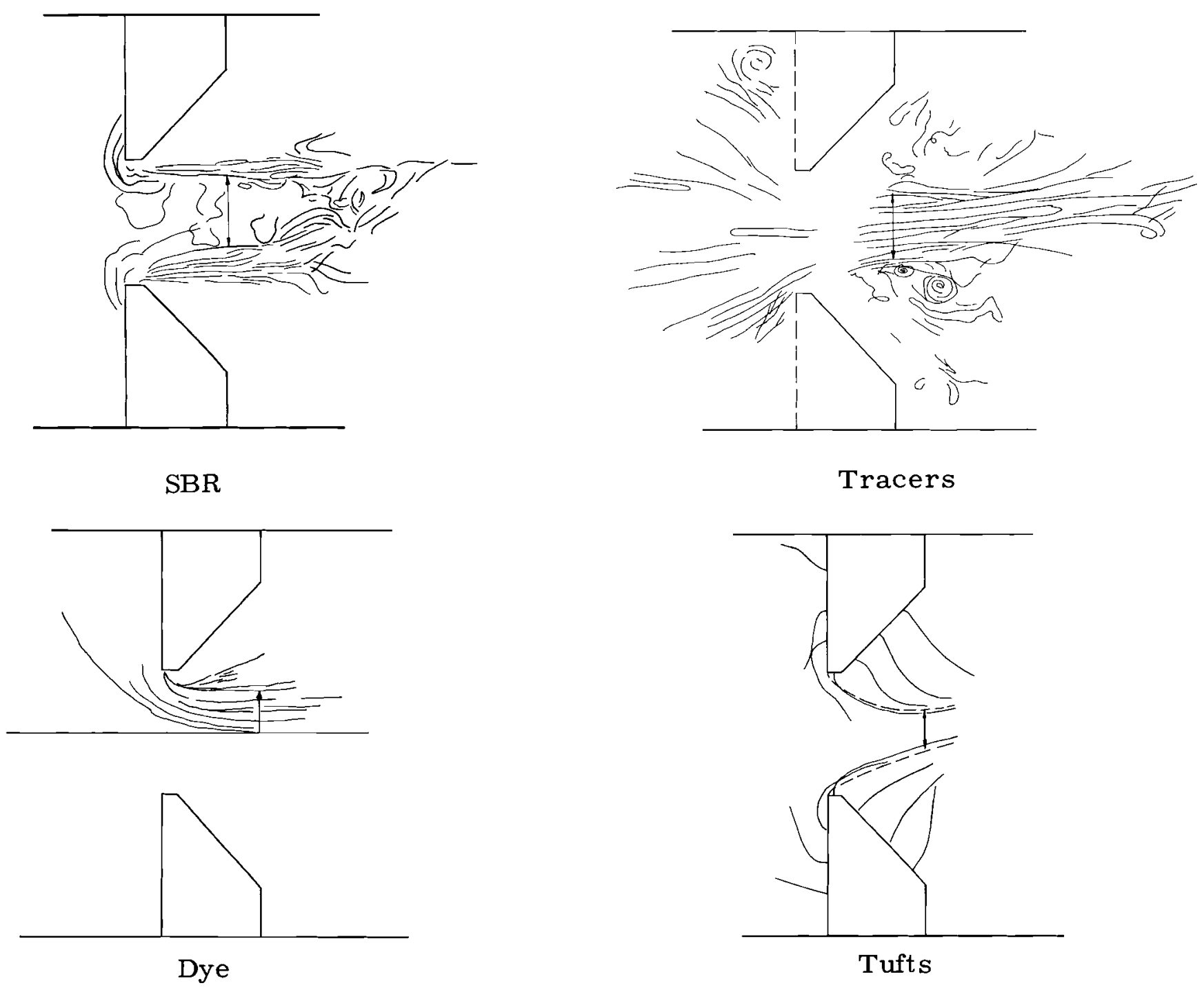

\section{FIGURE IX}




\begin{tabular}{|c|c|c|c|c|}
\hline \multirow{2}{*}{$\begin{array}{c}\text { Reynolds } \\
\text { Number }\end{array}$} & \multicolumn{4}{|c|}{$\begin{array}{c}\text { Apparent Thickness, Ta, } \\
\text { in inches }\end{array}$} \\
\cline { 2 - 5 } & SBR & Tracers & Dye (a) & Tufts \\
\hline \hline 935 & 0.305 & $(\mathrm{~b})$ & $(\mathrm{b})$ & (b) \\
\hline 1880 & $\begin{array}{l}0.350 \\
0.333\end{array}$ & $\begin{array}{l}0.420 \\
0.327\end{array}$ & $\begin{array}{l}0.389 \\
0.400\end{array}$ & 0.222 \\
\hline 3140 & 0.305 & $\begin{array}{l}0.300 \\
0.300\end{array}$ & $(\mathrm{c})$ & 0.293 \\
\hline \multirow{2}{*}{4690} & 0.305 & $\begin{array}{l}0.318 \\
0.308\end{array}$ & $\begin{array}{l}0.445 \\
0.455\end{array}$ & 0.318 \\
\hline
\end{tabular}

(a) Distance from centerline to streakline times two.

(b) Unable to achieve the required low flow for this Reynolds number.

(c) Streaklines failed to picture vena contracta due to improper placement of injection needle.

\section{FIGURE X}

Table of Apparent Thickness, Ta, of Vena Contracta

Figure XI shows selected pictures of the upstream corner of the orifice model. The circular pattern of the dye and tracers in the upper corner of these pictures clearly defines the eddies existing in these corners. Unfortunately, the upper corner of the SBR picture is obscured by bubbles but it is very similar to the pattern as shown in the bottom. The closed pattern observed suggests, perhaps, eddy motion - but it is certainly not obvious. The explanation of this may be associated with the integrating nature of the SBR isochromatics as compared with the point associated of streak and streamlines. Other explanations could be advanced, but the point is that the SBR method does not visualize these eddy patterns very well.

The wake behind a cylinder is shown in Figure XII. Note that the dye diffuses to obscure details of the wake while the tracer paths fluctuate 


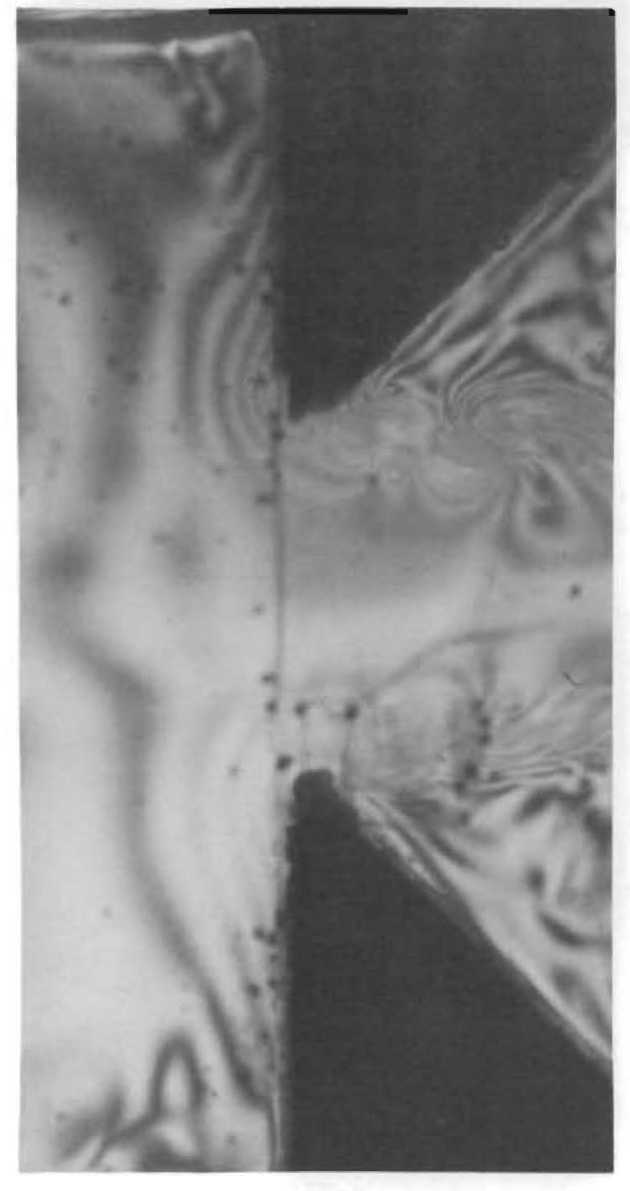

SBR

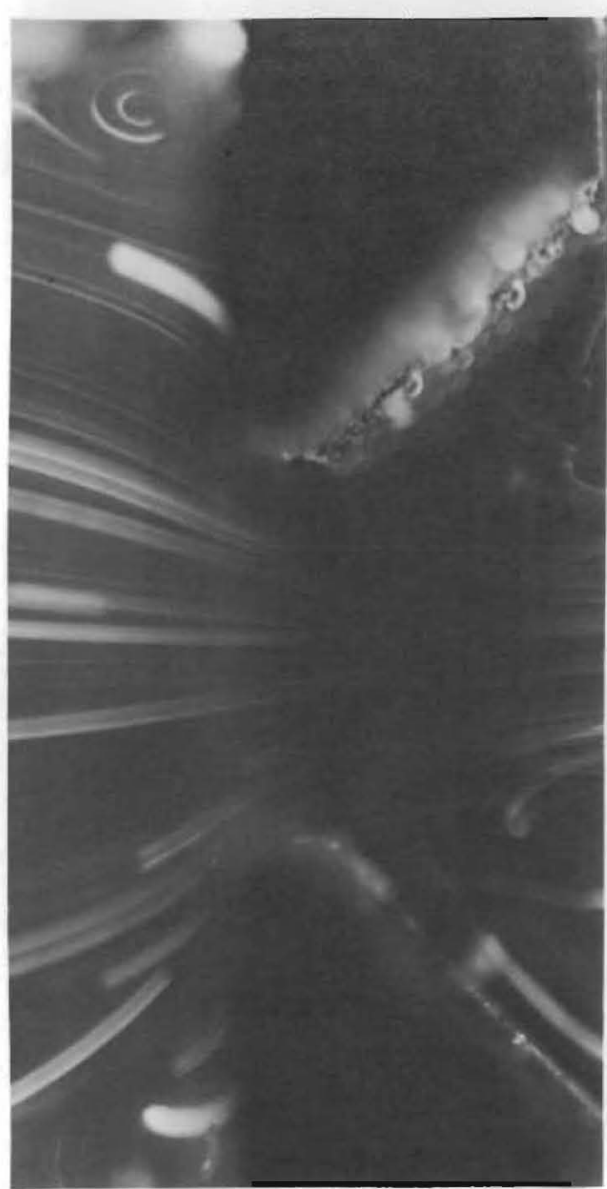

Tracers

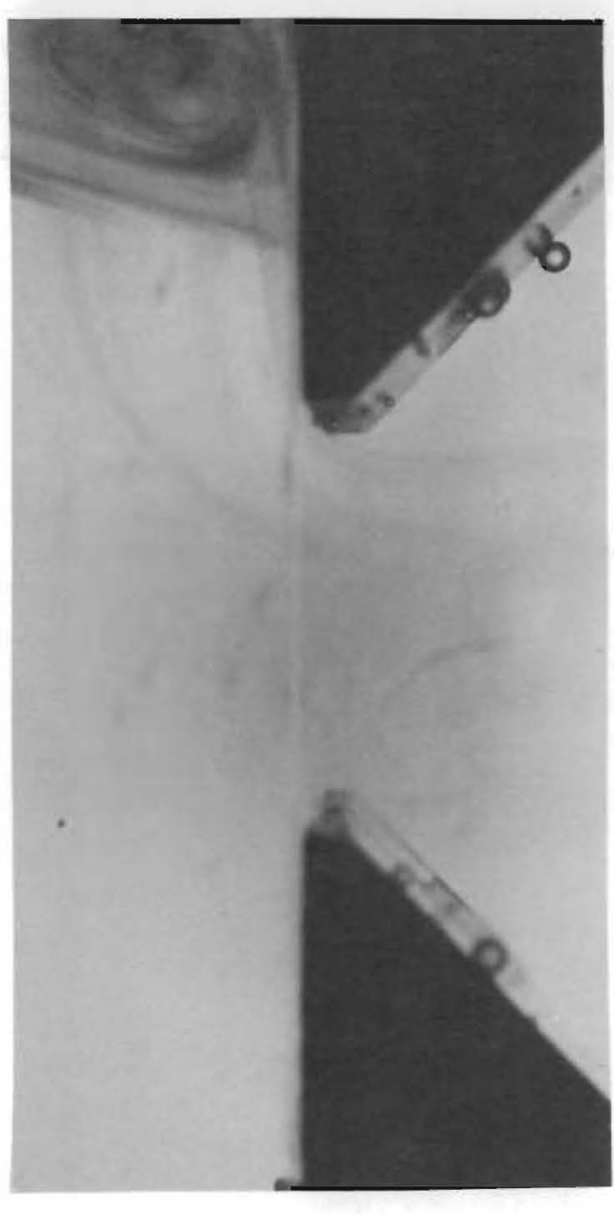

Dye 


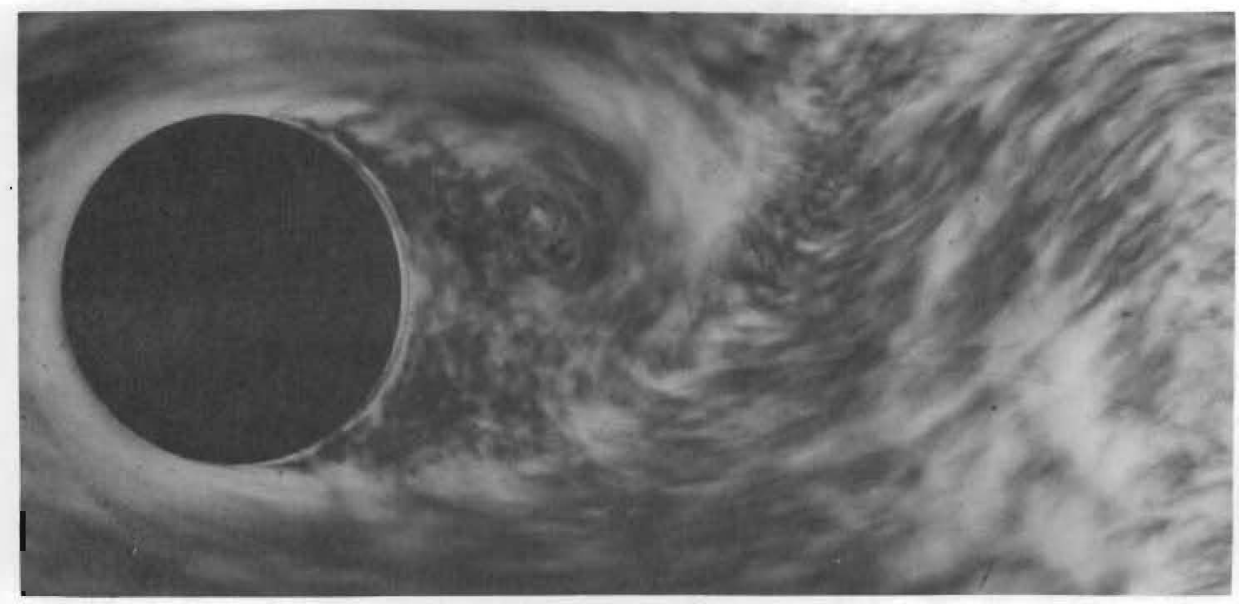

SBR

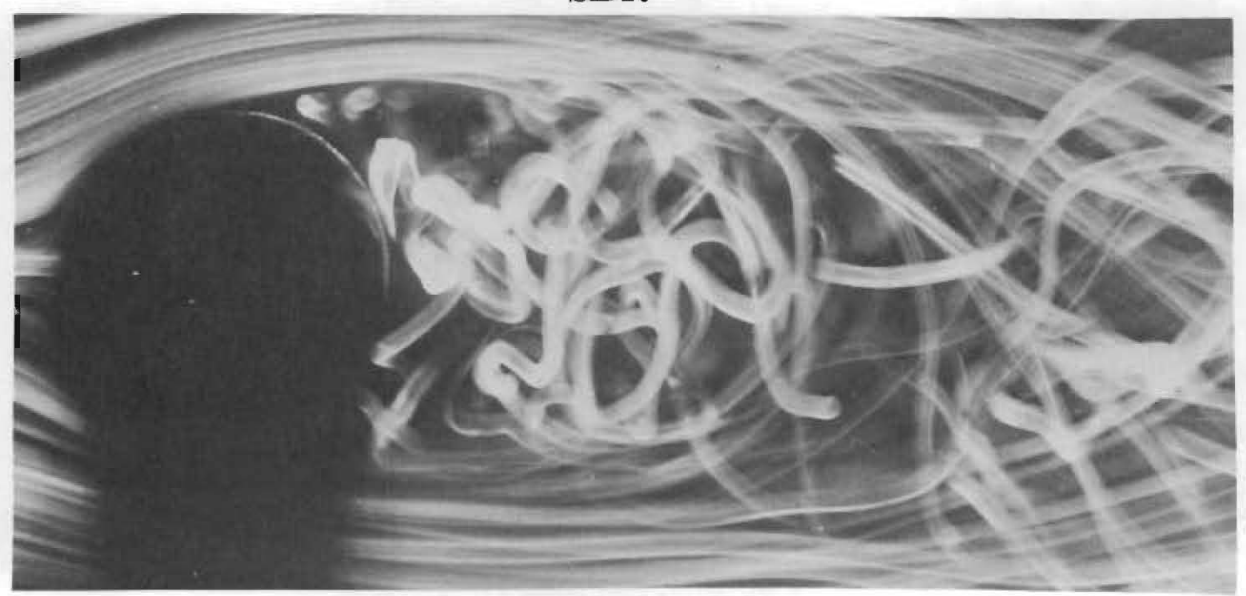

Tracers

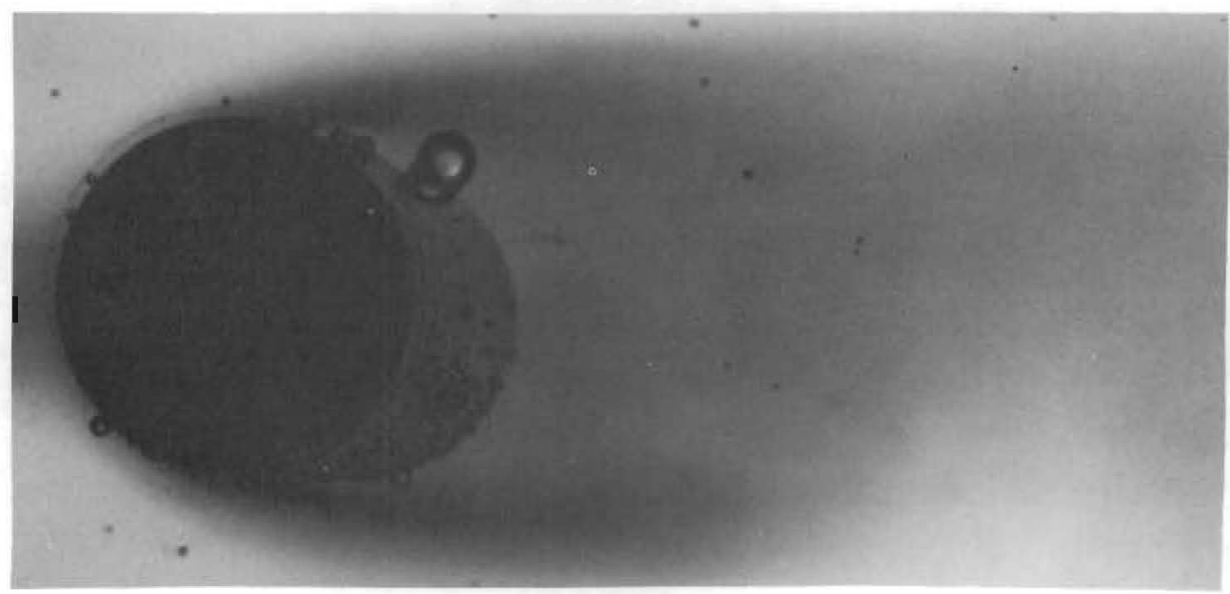

Dye

FIGURE XII

Cylinder Wakes 
so randomly within the wake that any characteristic fluid pattern formation is obscured. The SBR technique, on the other hand, produces a very clear picture with part of the wake phenomena which compares very favorably with photographs by Tietjens. ${ }^{(22)}$ Birkhoff and Zarantonello ${ }^{(10)}$ describe the growth and dispersion of the wake behind a cylinder in some detail. They state that "the wake swings from side to side." This effect was noted in the SBR tests and is clearly seen on high speed (approximately 2000 frames per sec.) motion pictures. High speed movies were not made of the dye and tracer techniques. Visually, it was noted that oscillations could be seen with the tracer path lines. It is not known how much of this is attributed to random particle motions and how much to the "swing effect." No side-to-side motion was detected with the dye. The tracers made visible the three-dimensional nature of the flow. This effect could not be photographed with our techniques but can be detected by blurring and sudden termination in some of the photographs.

Thus, the SBR technique does not produce pictures of clearly defined circular eddies but it does give a good visualization of both transient and frozen wakes.

General Observations. A number of general observations are included as a fourth area of evaluation of the SBR technique.

Flows were observed which had time independent isochromatic lines. These are regions of laminar fiow. See Figure XIII for a comparison of laminar and turbulent flows. On the forward edge of some models, at flows in the transition range, these stationary laminar isochromatics were observed to break down and re-form so that a regular oscillation was observed. It is believed that this is a case of localized intermittent turbulence similar to studies by Couette. (22)

The sequence photographs of Figure XIV gives a direct comparison between the velocity of a discrete isochromatic line and a plastic sphere. It is assumed that the sphere travels at the fluid velocity. Close observation of this set of photographs shows that any particular 


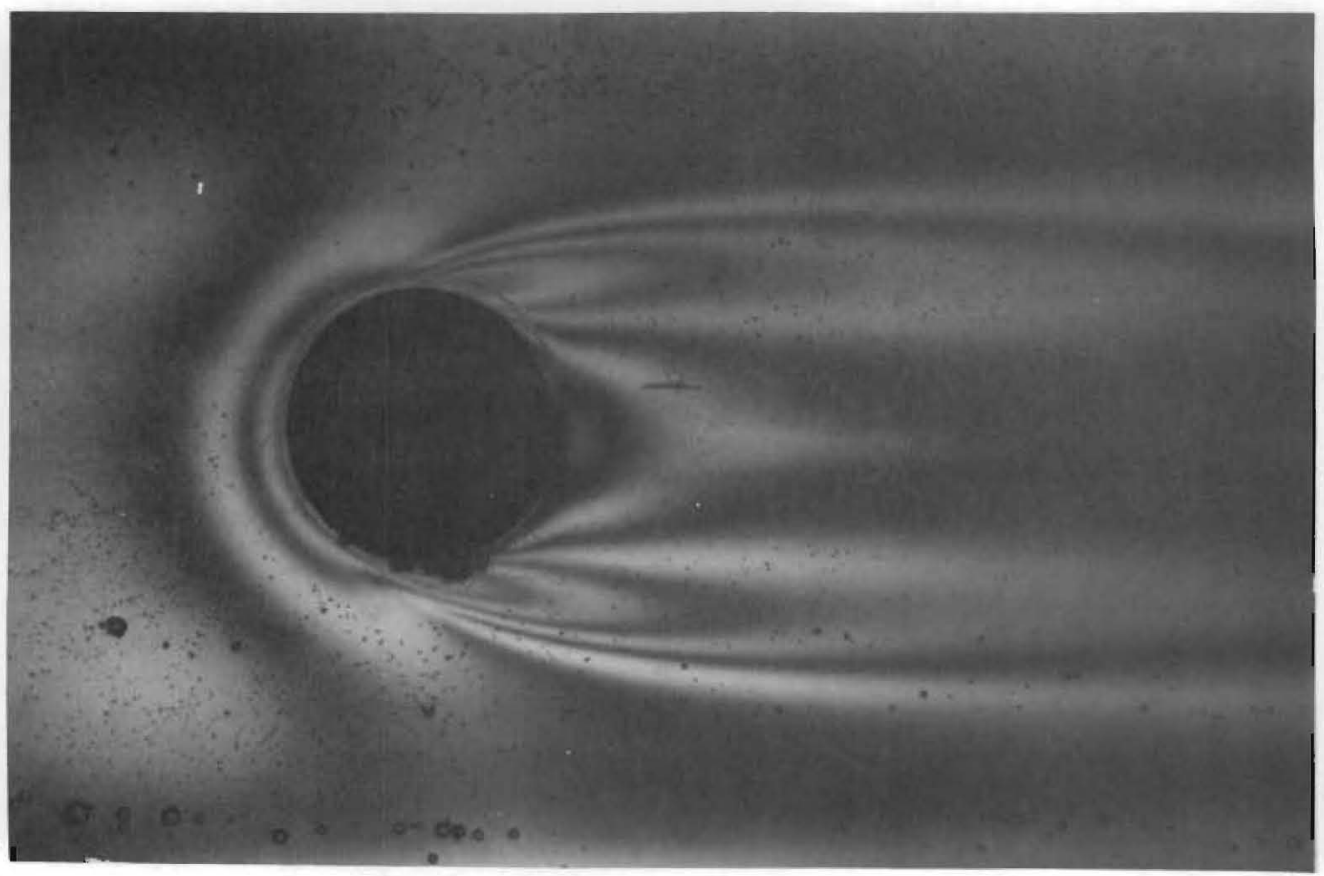

Laminar, Reynolds Number $=860$

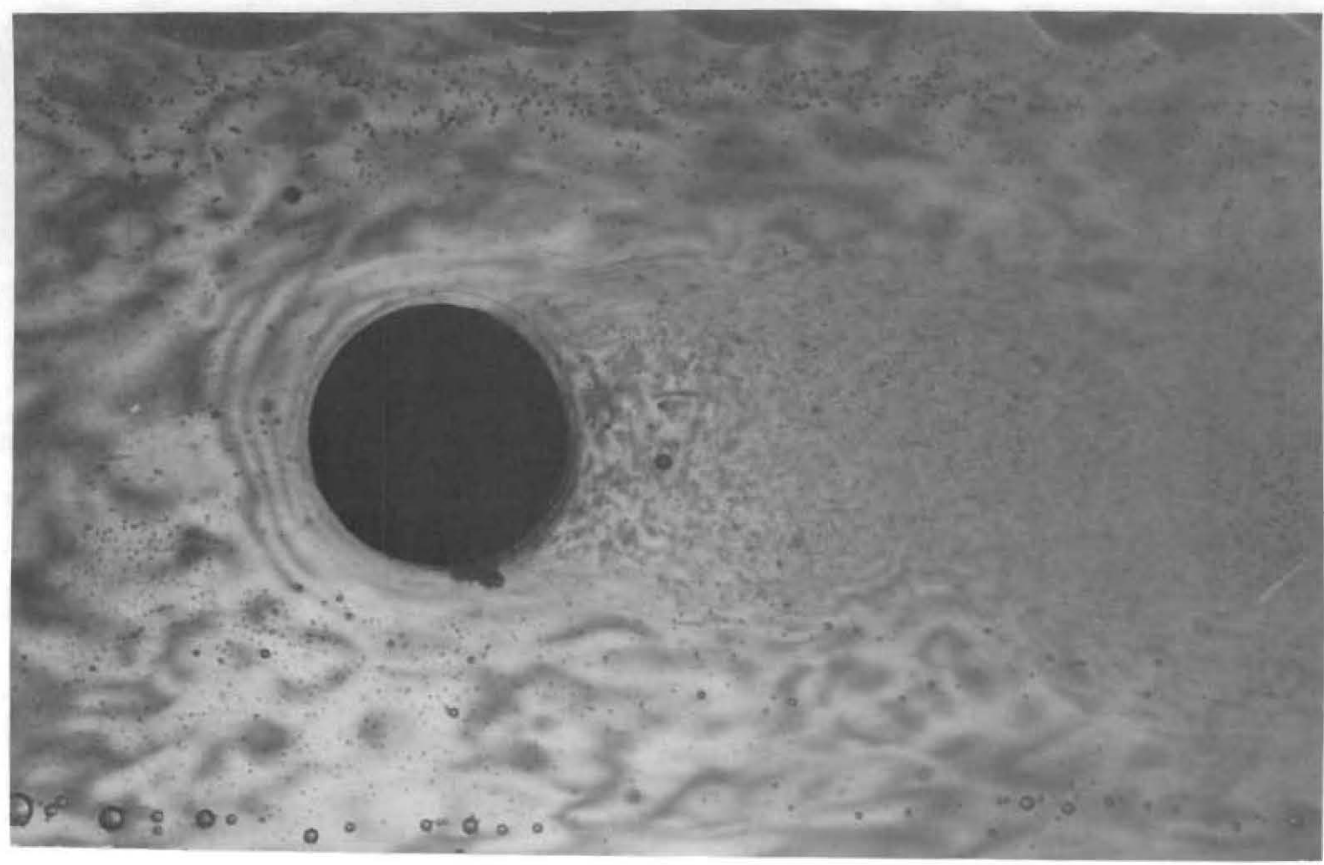

Turbulent, Reynolds Number $=12,000$

FIGURE XIII

Laminar and Turbulent Flows - SBR 

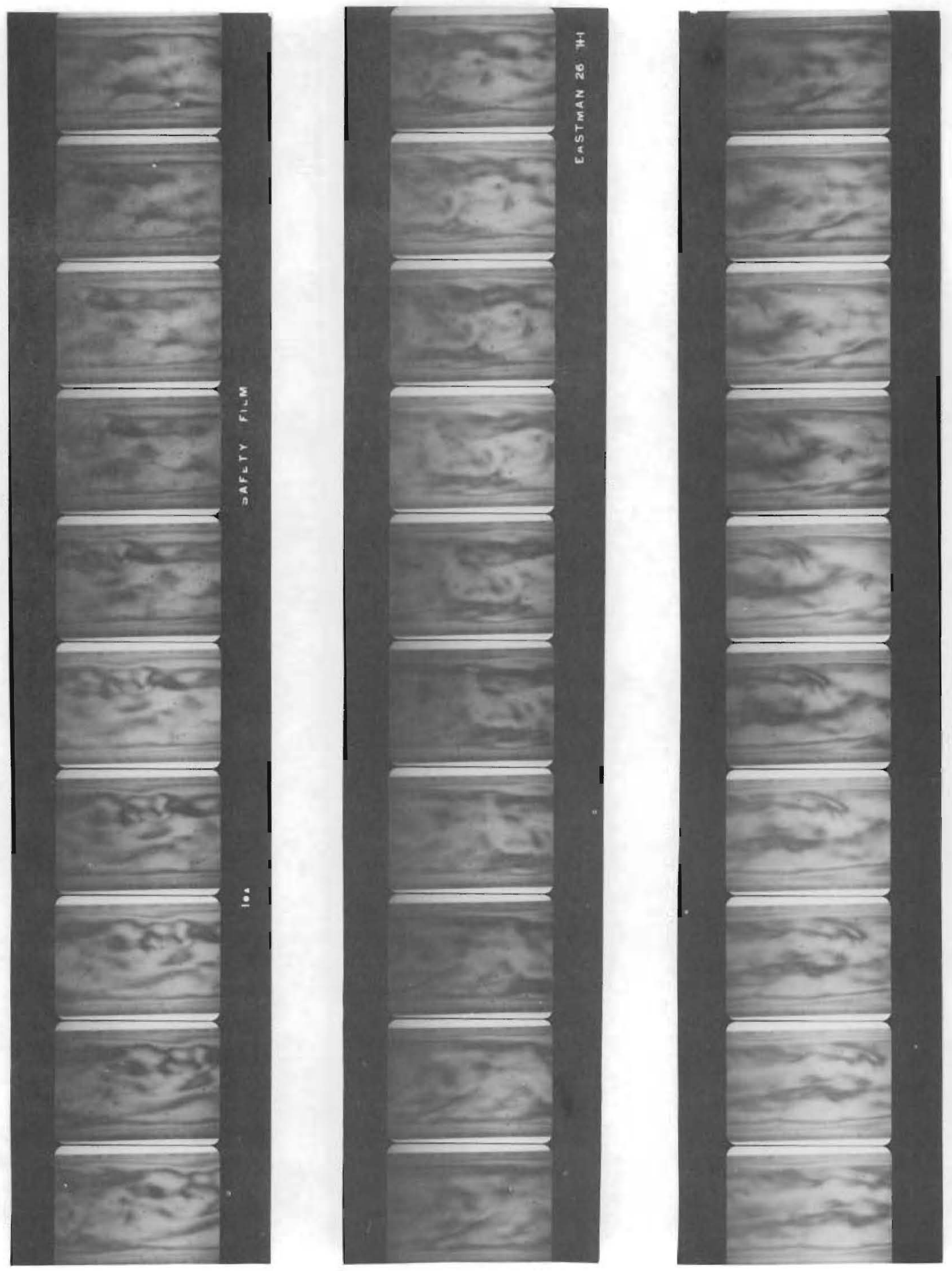

FIGURE XIV

Flow of Tracers and SBR

(Sequence: Top-to-Bottom, Left-to-Right) 
particle traverses the picture width in nine sequential frames. Any particular fringe pattern also takes nine frames. In addition, particles and fringes retain their relative space relationship throughout the nine frames. This leads to the conclusion that velocity of the isochromatic fringes is equal to the velocity of the fluid.

Observation of the high speed motion pictures gives a vivid impression as to the complex nature of fluid motion in turbulent flow. CONCLUSIONS

Based on the results of these tests, it is concluded that the flow visualization technique based on streaming birefringence has a definite potential for use as a fluid mechanics tool.

The SBR produced reasonable pictures of fluid flows to the extent of accuracy of this investigation. It is believed that quantitative evaluation of turbulent flow is extremely difficult, if not impossible. It should be recalled that the purpose of this investigation was a general comparison of a number of diverse flow conditions to see if further work with SBR visualization techniques would be useful.

Keeping in mind that more detailed and restrictive tests with finer measurements are required to explain the variation of certain results, it is concluded that further work with SBR visualization is justified.

The conclusion that the SBR technique can be used as a fluid mechanics tool does not imply that it is superior to the other techniques. It does mean, however, that it can be considered, at the very least, as competitive with them. The decision concerning the best fluid visualization tool must be based on the requirements of the investigation itself.

Limitations and Remarks. The method of fluid visualization by the SBR techrique has a number of limitations. It involves concepts from fluid mechanics, optics, colloidal chemistry, similarity theory, and rheology. Some areas have unresolved problems. An example is the absence of an accurate theory linking colloid particle motion to observed 
birefringence. Initial familiarity and utilization of these concepts tends to make the SBR visualization method complex and hence affects its choice as a laboratory tool.

The handling and preparation of the bentonite solution is not easy and involves procedures which are not standardized. Many of the techniques used border on the "artistic", with some investigators (24) reporting problems serious enough to develop a new SBR fluid and others (2) reporting good performance when proper clay was used. See Appendix E for details.

These physical problems, coupled with the fact that turbulent pictures do not resemble the idealized streamline flows, has contributed to the lack of popularity of this technique.

However, the fact that streakline and pathline visualizations approximate, but do not necessarily coincide with, idealized streaklines is too often not appreciated. The pathlines obtained in these studies and the strong appeal by Hanna ${ }^{(25)}$ to use caution in interpreting flow phenomena based on streakline visualization are cases in point.

Since well behaved streamlines do not really exist in turbulent flow, since pathlines always produce streaked pictures of some type, and since streaklines often diffuse and wander, there is strong reason to believe that visualization of turbulence of SBR methods might more accurately reflect many aspects of nature of turbulent flows.

The fact is emphasized that the purpose of this study was to compare the characteristics of different flow visualization methods, and this has been accomplished.

Very preliminary runs at turbulent flows with three-dimensional models, suggest that this area of investigation might also lead to fruitful results. 
APPENDIX A

FLOW VISUALIZATION 


\section{APPENDIX A}

\section{FLOW VISUALIZATION}

\section{INTRODUCTION}

The use of techniques which make dynamic fluids visible to the human eye has been an integral part of fluid mechanics for some time. Its importance has been demonstrated for fundamental scientific research and as a design aid and engineering tooî - from the very earliest beginnings to the immediate present - from the dye streamers used by Osborn Reynolds in 1883 in his investigation of turbulence to Schlieren photographic analysis of advanced missile designs in a modern, complex wind tunnel facility.

The use of flow visualization implies three questions:

1. Why are visual studies used?

2. What information can be expected from visual studies?

3. What visual study techniques are available?

\section{IMPORTANCE OF VISUALIZATION METHODS}

The utilization of visual methods and techniques has enabled investigators to predict, analyze, calculate, and design fluid flow regimes and equipment. It is a tool utilized in all of fluid dynamics, from use as a basic research aid to use as an engineering tool.

In his opening remarks to the Symposium on Flow Visualization, Kline ${ }^{(26)}$ clearly points out why this is the case. He notes that in over 200 years, only a handful of exact solutions are available to the governing differential equations of fluid mechanics: Navier-Stokes equations, continuity equation, equation of state, and the First and Second Laws of Thermodynamics. Moreover, these hold only when the governing parameters are within narrow limits. Also, different conditions and boundary values can produce different solutions. 
For instance, the simple example of incompressible flow around a cylinder has shown to have five distincly different flow patterns in the Reynolds number range of one to $10^{6}$. Only one, or perhaps two, of these cases is predictable by acceptable theory and only one transition can even be attempted by currently available analytical methods, and this involves some questionable assumptions.

Or consider the transition from laminar to turbulent flow in a circular conduit. Reynolds, as is well known, reported the existence of this transition over 80 years ago, with the aid of a visual study. During this time some of the ablest applied mathematicians have expended considerable, complicated, and sophisticated effort on the problem of laminar-turbulent transition. Only within the last 15 years has the way been opened for calculation of friction and heat transfer in this transition region.

Or conversely, the potential flow calculation demonstrating that no lift can be generated on an airfoil in a flow without friction probably retarded progress toward powered flight by almost 50 years. There are other examples where mathematical theory alone supplied the wrong answer by assuming the wrong flow model.

Adequate data on flow patterns is required for all good fluid mechanics work. Thus, to answer our question, "Why are visual studies used?", it is seen that visual studies are oftentimes the cheapest, quickest, and most effective way to obtain this data.

EXPECTED RESULTS

A consideration of those flow examples which are now relatively manageable reveals two factors which are always present. First, a clear, well verified relationship between the flow regimes and the numerical range of the governing non-dimensional parameters. Second, good data and/or adequate basis for analysis are available by which the performance characteristics, and their rate of change, with respect to the governing parameters can be expressed for each flow regime. 
The establishment of a flow regime alone is not enough. The ability to predict occurrence as a function of numerical values of the governing parameters is required. Conversely, an analytical solution without demonstration that the calculated result is the same as the actual flow pattern is of very limited value. Thus, for optimum engineering work the joining of these two techniques is desired.

There are, however, a large number of cases in actual fluid flow practice where the flow is so complicated that analytical treatment is difficult or impossible. Very often, the skilled use of associations between flow characteristics and controlling parameters will produce, in itself, good or even excellent engineering designs.

Examples of this abound. For instance, the inclusion of vanes in a short turn heat exchanger by Frey ${ }^{(26)}$ to reduce flow losses.

The work by Leaf ${ }^{(27)}$ in determining, evaluating, and correcting the cause of excessive firebox and flue erosion on steam locomotives is another example.

Also, the extensive work of Chesters and Philip (28), Howe and Philip ${ }^{(29)}$, Halliday and Philip ${ }^{(30)}$ and Collins and Tyler ${ }^{(31)}$ on the interpretation of complex flows in open-hearth steel furnace models is a good example of a complete study based solidly on visualization techniques.

Nor are all examples in areas normally considered as hydraulics, as is demonstrated by the study of geophysical fluid models for weather prediction by the Navy. (32)

The above discussion thus shows the range of information that can be expected from visual studies. METHODS AVAILABLE

It is presumptuous to attempt a listing of the very large number of techniques available to make fluid phenomena visible. The following list, therefore, is representative, only. 
1. Dyes - various types have been used, introduced in a variety of ways.

2. Particles - these include such things as sawdust, plastics, and special mixtures of wax, lead, and other materials. The more spherical they are the better they reflect light.

3. Tufts - sometimes fastened to the model and sometimes on a traverse rod.

4. Electrochemical bubble - includes such things as fine hydrogen bubble generation on very small diameter wires. An extremely intriguing and promising method.

5. Wall Trace Methods - the placing of patterns on boundarys by smearing or discoloring fresh paint, or by chemical stain, etc.

6. Surface Tracers - the use of fine aluminum powder on an especially high quality water surface is an old and powerful technique.

7. Streaming birefringence.

For compressible fluids:

1. Smoke and chemical cloud streamers

2. Shadowgraph

3. Schlieren, and other related techniques

The methods fall into four general classifications (33),

(a) Contamination of the fluid with foreign matter of contrasting color or index of refraction.

(b) Small indicators which pivot to indicate flow direction.

(c) Fluidynamic - optical effects.

(d) Tracing of boundary flow by erosion or staining.

These, then, constitute the field of available techniques for fluid visualization. 
APPENDIX B

ESSENTIAL OPTICS 


\section{APPENDIX B}

\section{ESSENTIAL OPTICS}

Birefringence is the phenomenon of light ray reinforcement and interference as these rays pass through certain types of materials. This appendix outlines those optical properties upon which the phenomenon of birefringence is based. For a more complete coverage, any good book on photoelasticity should be consulted. A good introduction is readily obtained from Hendry ${ }^{(34)}$ or Jessop-Harris ${ }^{(35)}$. While books by Frocht $^{(36)}$ and Coker-Filon ${ }^{(37)}$ give more comprehensive treatments.

\section{PROPERTIES OF LIGHT}

The electromagnetic and quantum mechanic theories of light are presently held as the most popular. However, in the study of birefringence, since we are concerned only with the passage of light through transparent materials, we shall consider light as a simple transverse wave.

Consider a point in space from which light is being radiated in all directions. After a period of time the wave distribution will have reached positions which form a sphere around that point. A line connecting the center to surface of this sphere is defined as a "ray". If the time interval is sufficiently large, the sphere can be treated as a plane surface and two neighboring rays considered as parallel.

An interface between two translucent media will cause a bundle of these parallel, incident rays to both reflect and refract, assuming that the incident light intersects the plane at an angle. This is seen by noting Figure XV. The plane surface normal to the rays is called the wavefront. As the incident light falls upon the interface, in the plane of the paper, part of the rays are refracted at angle $\mathrm{R}$ giving rise to the refracted wavefront at time $t+\Delta t$. Since the velocity of light in the second media is slower, at time $\Delta t$ the initial refracted rays are not as long. A bending or refraction at an angle $r$ from the normal to the surface, therefore takes place. 
BNWL-31

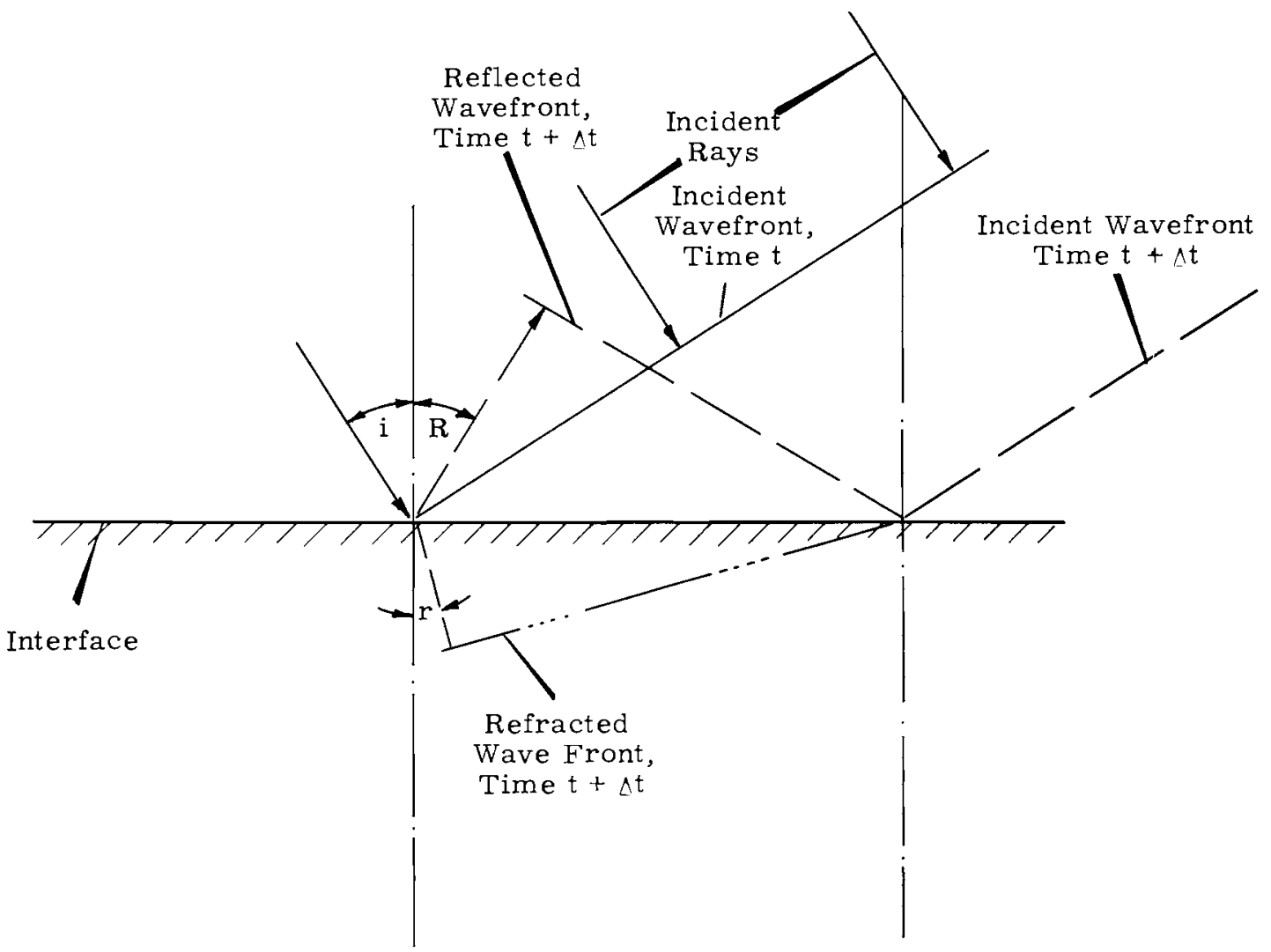

FIGURE XV

Reflection and Refraction at a Boundary

The refractive index, $\mu$ is defined by:

$$
\frac{\sin i}{\sin r}=\frac{\text { Vel. of light in the first media }}{\text { Vel. of light in the second media }}=\mu
$$

All angles are measured in a single plane, known as the "plane of incidence". This plane is normal to the interface at the point of incidence.

Light can be considered as electromagnetic wave radiation originating from a source, similar to an electromagnetic radio wave originating from an antenna. In the case of the radio wave coming from one vertical antenna, the wave will oscillate in one plane, the vertical, and can be thought of as "vertically polarized". Light waves from normal sources--the sun, light bulbs, etc, ,--come from submicroscopic oscillators which are completely random in orientation. Thus, ordinary light 
can be considered as oscillating in all directions or non-polarized. Hence, plane polarization is defined, for our purposes, as the restriction of light wave vibrations to a given plane.

PROPERTIES OF TRANSPARENT MATERIALS

Optically isotropic materials are those which transmit light with an equal velocity in all directions. Examples include air, pure water, and most glass. Anisotropic materials, on the other hand, have the property that light is transmitted through them with a velocity dependent on its direction of travel. Examples of anisotropic media include crystals, most plastics, and stressed glass.

Consider a point, $\rho$, within a crystal, from which light is radiating, as shown schematically by Figure XVI-a. Two wave fronts will be propagated from this point. One set will be spherical, as in the case of the isotropic media; the other set will be ellipsoidal. The spherical set is known as an ordinary wave while the ellipsoidal set is known as the extraordinary wave.

If the crystal is held fixed and sliced along the dotted line A-A, and light directed normal to this cut surface, two distinct wave fronts will be propagated through this crystal as shown in Figure XVI-b. Likewise, if the crystal is sliced along the dotted line B-B and light directed normal to this face, only one wave front will be propagated as shown in Figure XVI-c. And, in general, if the crystal is sliced at any angle, components of the two wave fronts, as shown in Figure XVI-d, will result.

In addition to this effect of retardation of the extraordinary ray over the ordinary ray, a selective polarization process results. That is, the extraordinary ray is plane polarized at right angles to the plane polarized ordinary wave. This relationship is shown schematically in Figure XVII. This effect was first noted in 1669 by Bartholinus who observed two images through a piece of calcite crystal. This birefringence, or double refraction, lent considerable weight to the support of 
Huygens wave theory of light as opposed to the previously held corpuscular theory.
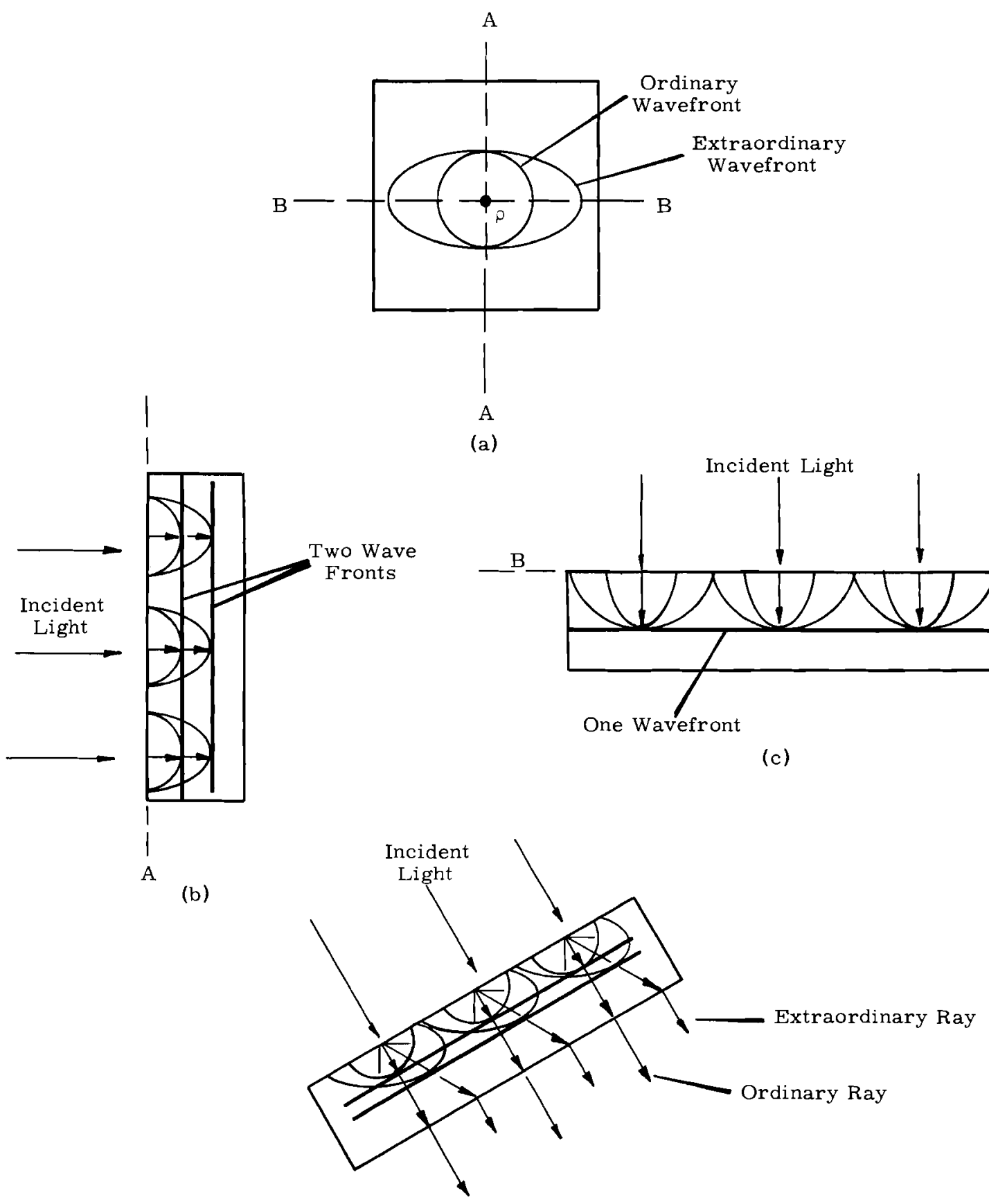

(d)

FIGURE XVI

Crystal Transmittance of Light 


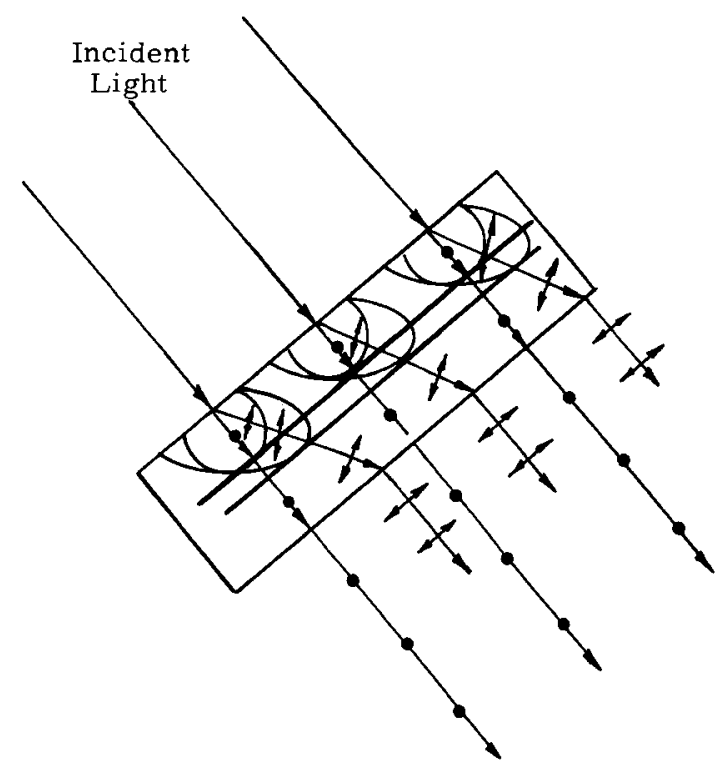

Crystal orientation as shown in Figure XVI-d
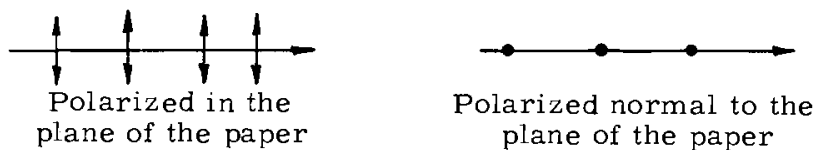
plane of the paper

NOTATION

FIGURE XVII

Birefringence

\section{INTERACTION OF LIGHT WAVES}

A plane polarized light wave can be considered as a vector quantity as illustrated in Figure XVIII-a. If two waves at right angles and slightly out of phase are summed vectorially, as shown in Figure XVIII-b, the resultant wave shape is similar to Figure XVIII-c, and is called an "elliptical" wave.

Since ordinary light is composed of vibrations in many planes, passage of this light through a crystal results in many elliptically oriented vibrations and is indistinguishable from ordinary light. Thus, to study 
the internal effects of an anisotropic material by analyzing the emergent light, we must start with an incident light which has a controlled vibrational orientation.

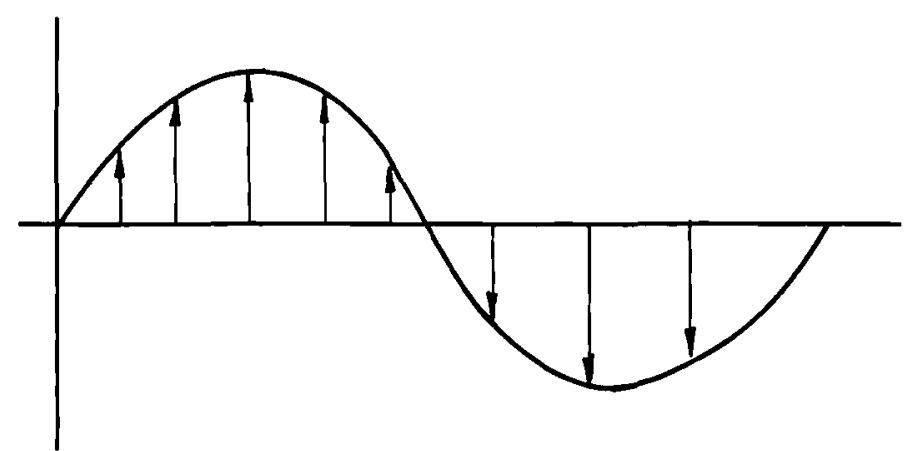

(a)

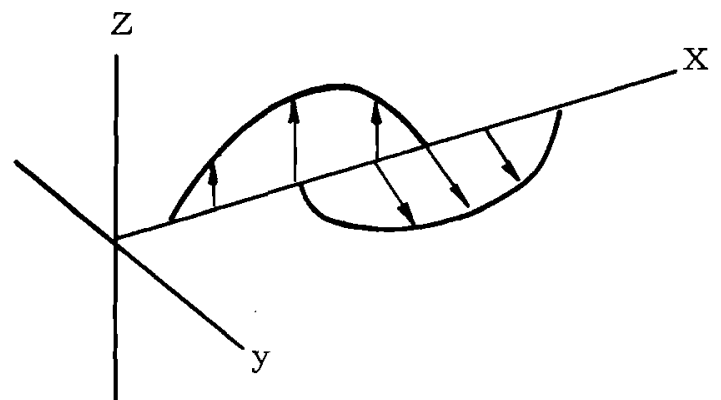

(b)

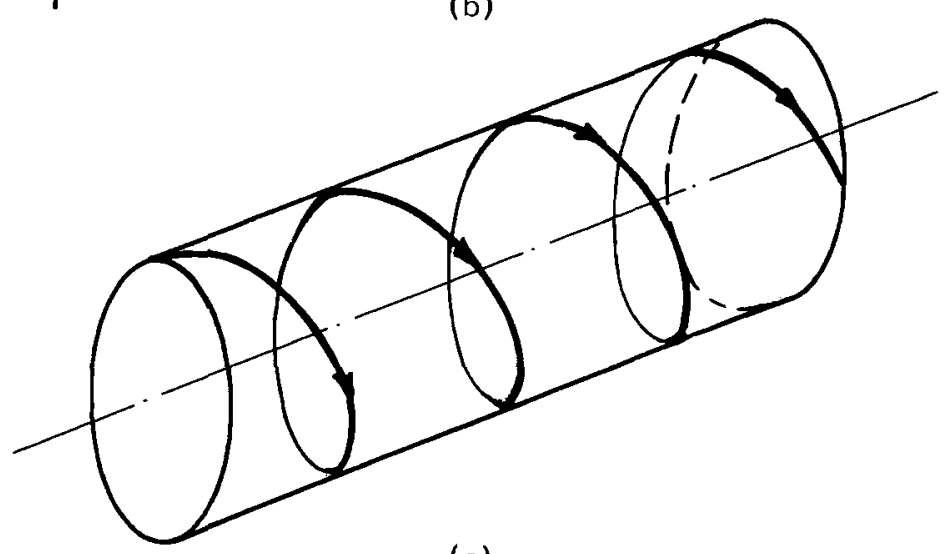

(c)

FIGURE XVIII

Wave Interaction 
If a crystal plate is made a proper thickness to produce a relative retardation of $1 / 4$ of the incident light wavelength and if the waves are of equal magnitude, the plate is known as a "quarter wave plate" and the emergent light is "circularly polarized, " a special case of the "elliptical" form.

The property of wave interference is felt to be sufficiently well known as to require no elaboration here. However, one must keep in mind that the phenomenon of interference takes place only when the two vibrations are in the same plane.

A polaroid film is a commercial dichoric product which absorbs all vibrations except those in a given plane, the plane of polarization. A plane polariscope is made by first mounting two polaroids a short dis tance apart. The placement of a light source on the axis of the two polaroid plates completes the apparatus. The first plate is called the polarizer and the second is called the analyzer. The position of the analyzer in reference to the polarizer determines the amount of transmitted light. If the two are parallel, full intensity is transmitted as schematically shown in Figure XIX-a. If the two are "crossed" at right angles, complete blocking is experienced, as is indicated in Figure XIX-b.

If two quarter wave plates are added, one placed after the first polaroid, and one before the second polaroid, a circular polariscope is obtained. It is so named because the nature of the light existing between the polarizer and the analyzer is circularly polarized, as shown schematically in XIX-c.

POLARISCOPE ACTION

Now using the aforementioned principles, consider a crystal between the polarizer and the analyzer of a plane polariscope. The polarizer reduces the ordinary light to polarized light of a single plane which is incident upon the crystal. Due to birefringence, an elliptical wave emerges from the crystal. This elliptical wave pattern is the equivalent to two polarized waves oriented at right angles and with a certain relative 
retardation between them. The analyzer reduces these two waves to components oriented in the same plane. These two components may now interfere, depending upon the orientation of the analyzer, to produce either dark or light bands. For instance, if the polariscope is adjusted to produce a dark field and a crystal added whose thickness is such as to produce a relative path retardation of an odd number of half wavelengths, the resulting effect on the analyzer will be restoration of light corresponding to the crystal shape against the original dark background.

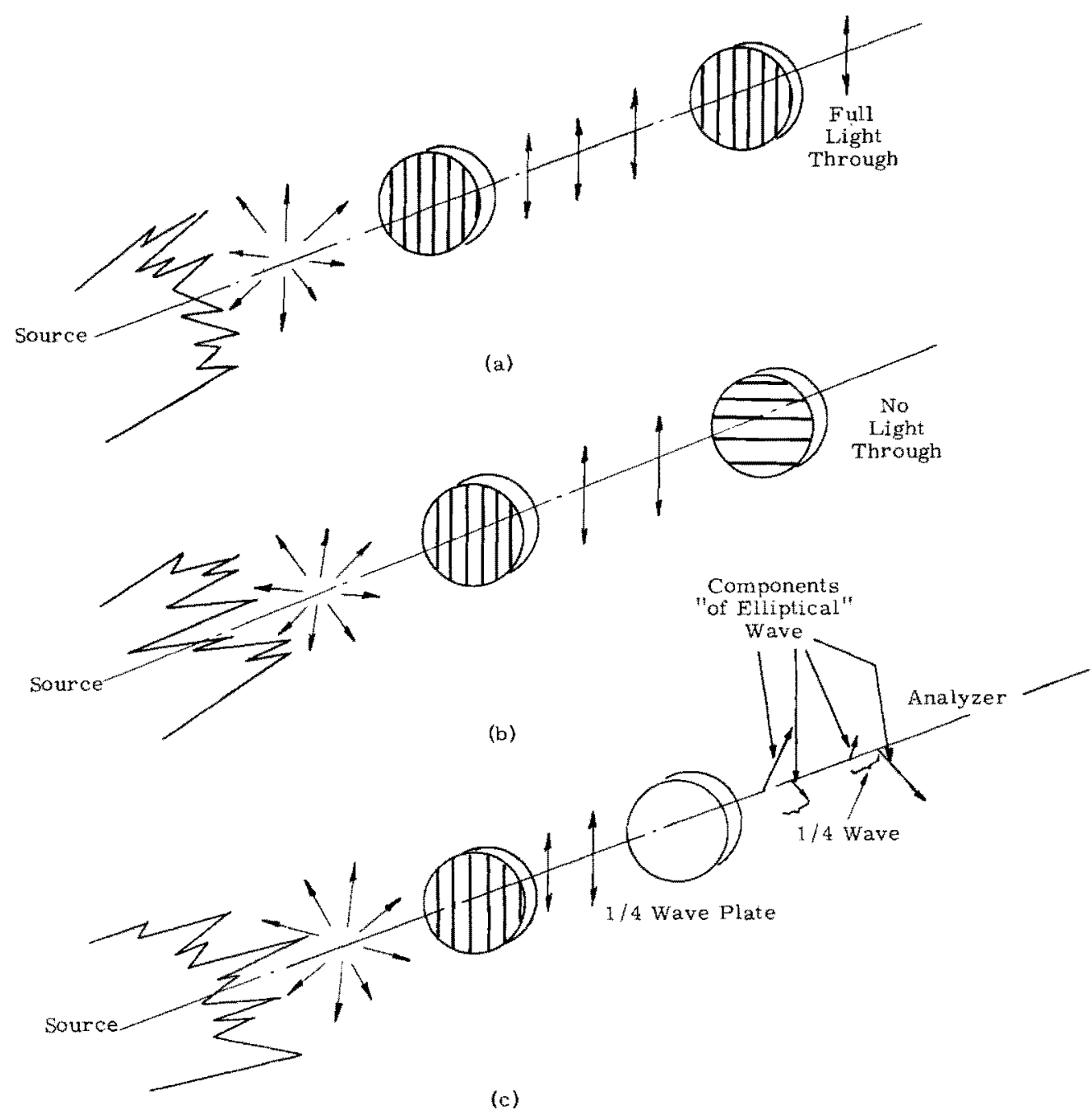

FIGURE XIX

Polariscope Operation 
A circular polariscope operates in the same way and the appearance of the specimen is the same except that the crystal orientation does not affect the intensity of the light transmitted.

\section{PHOTOELASTICITY}

The science of photoelasticity is based on the fact that certain transparent materials, such as plastics, behave as an isotropic media under no stress and as an anisotropic media when stressed. A light wave entering such a stressed material is emitted as two waves polarized in the directions of the principle stresses. The magnitude of relative retardation is directly proportional to the difference in the principle stresses and to the thickness of the sample.

Using plane-polarized light, the locus of points where the principal stress direction coincides with the polarization axis will appear as dark bands and are known as isoclinic lines. Thus, it is seen that the use of circularly-polarized light, because effects of sample orientation are eliminated, will eliminate the isoclinics and transmit only the isochromatics.

The theory of photoelasticity is complete and this discipline has been very effectively utilized as an analysis tool for stresses in solid bodies.

\section{STREAMING BIREFRINGENCE}

Streaming birefringence is based on the fact that certain fluids are isotropic at rest and are anisotropic while flowing. It has been demonstrated that the amount of retardation is a function of the rate of strain and the orientation of the streamlines. Unfortunately, the theory of SBR is not well established and the analysis techniques required for effective evaluation is, at the present time, in a state of development. Appendix E discusses SBR histories and theories in more detail. 


\section{APPENDIX C \\ PHOTOGRAPHIC EQUIPIENT AND METHODS}




\section{APPENDIX C \\ PHOTOGRAPHIC EQUIPMENT AND METHODS}

The primary method of data recording for these tests was by photographs. Single photographs of all four techniques were used for all of the comparisons made. Two additional techniques, sequence photographs and high speed movies, were used with the SBR fluid.

The SBR flows were photographed with back lighting through the polarizer and analyzer, the dye and tufts with diffused back light, and the tracers with a vertical light slit and a horizontal camera.

\section{SINGLE PHOTOGRAPHS}

A $4 \times 5$ Linkof camera was used for the single SBR photographs and all of the other three techniques.

For proper lighting of the SBR fluid, a Stroboflash unit with an auxiliary flash pack capable of flash durations of $1 / 1400 \mathrm{sec}$. was used.

Two 500 watt photospot lamps were used for tracer illumination. These lamps were mounted in a special lamp holder which contained a narrow slit. The slit was 12 inches long, 8 inches high, and $1 / 4$ inch thick. The holder straddled the flow channel holding the $1 / 4$ inch thick, semi-collimated beam at the channel center. Air was blown through this slot to prevent overheating of the holder and to reduce the heating effect on the flow channel lucite. Exposure times were $1 / 5 \mathrm{sec}$, $1 / 2$ sec., and 1 sec., depending on the model and particular velocity of the fluid during that test.

The tufts and dye were photographed using a diffused backlight. Timing of the dye shots was critical since the patterns were continually changing and the desired effect had to be "caught" by the photographer. For example, it appeared that a velocity profile was made visible by injecting a large "slug" of dye and allowing the clear fluid to displace it. Four attempts to photograph this, however, were completely unsuccessful. 


\section{SEQUENCE PHOTOGRAPHS}

The sequence photographs of Figure XIV were taken with a $35 \mathrm{~mm}$ instrument recording camera, Model G-1, made by Automax. A High Speed Stroboscope, Type 501, made by Edgerton, Germeshausen, and Grier, Inc., was triggered with a microswitch inside the camera. The camera indexed at 16 frames per sec. and the effective shutter speed was 1 microsecond. The camera shutter was removed to minimize synchronization problems between shutter and flash. A synchronizing delay circuit was built to do this same job, but was not used.

\section{HIGH SPEED MOVIES}

A series of $50 \mathrm{ft}$. rolls of $16 \mathrm{~mm}$., motion pictures were taken of the SBR fluid with a Kodak High Speed type camera. Full speed was used, approximately 2000 to 2400 frames per second. The lens opening was $\mathrm{f} 2$ with three 500 watt spot lights located approximately 2 feet behind the polarizer. 
APPENDIX D

DESCRIPTION OF EQUIPIMENT 


\section{APPENDIX D}

\section{DESCRIPTION OF EQUIPMENT}

The equipment used in this study is shown in schematic form as Figure XX.

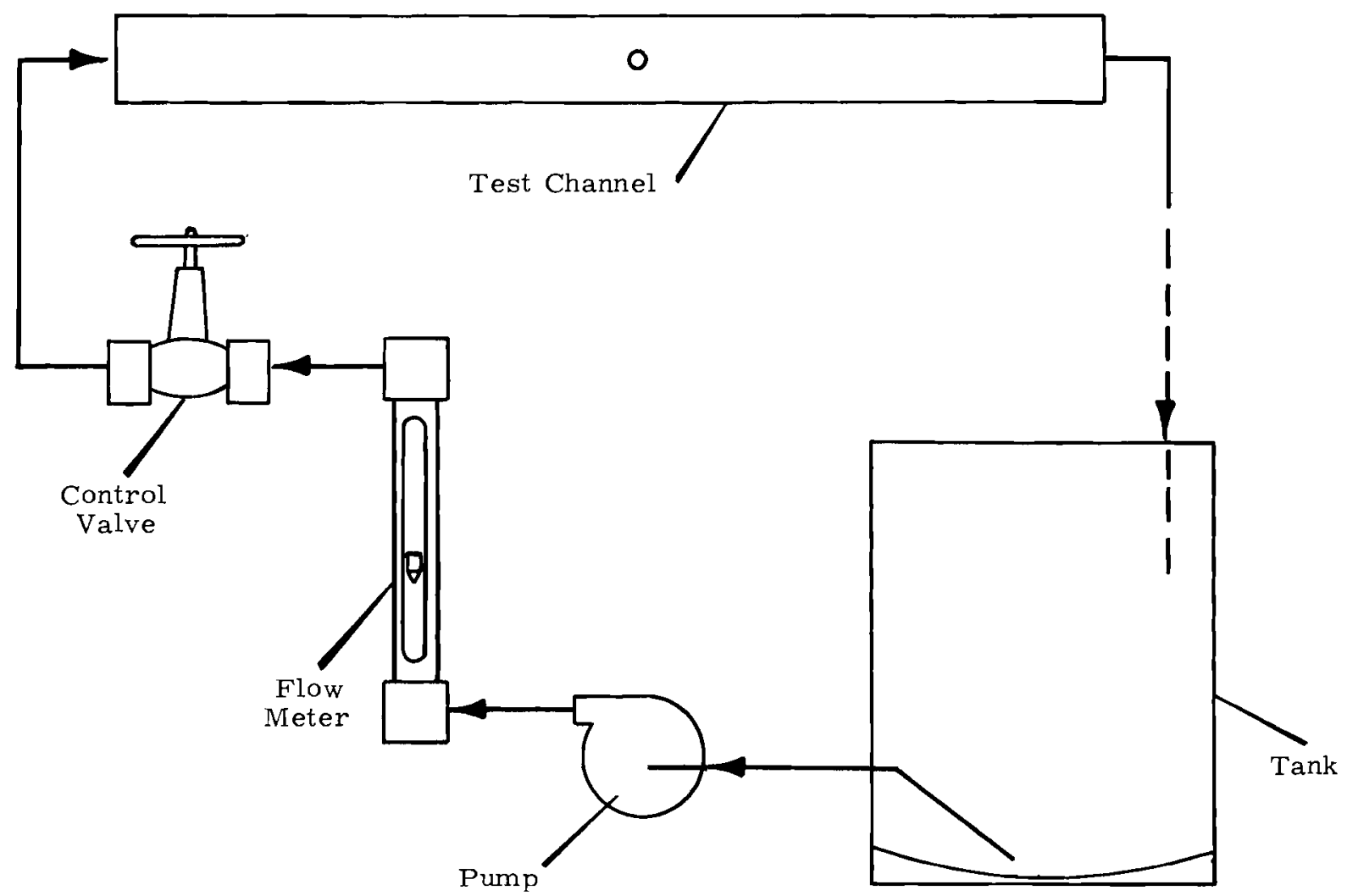

FIGURE XX

\section{Schematic of Flow - Flow Facility}

The facility is simply a recirculating loop which has a tank for storage, a centrifugal pump to move the fluid, a rotometer to measure flow, a valve to control the fluid, and a test channel for mounting models and viewing results.

All portions of the recirculating facility in contact with the working fluid are made of either stainless steel or plastic to reduce the tendency of the SBR fluid to gel. 
Provisions were included in the mounting stand for holding the polarizer and analyzer portions of a circular polariscope. The light bulb and reflector portion of the polariscope could be swung to one side to allow positioning of the required photographic light sources, as outlined in Appendix C.

In addition to the equipment associated with the flow facility, a supercentrifuge necessary in the preparation of a bentonite solution and a rotating cylinder viscometer were used.

The following is a brief description of the various equipment items. FLOW CHANNEL AND MODELS

The flow channel was made of $3 / 8$-inch thick lucite sheet, was 2 -inches square on the inside, and was 6-feet long. Chemical welds were used throughout. Square blocks at both ends were tapped to accept the stainless steel pipe threads. A short section of wall was removed near the center of this channel.

Four pieces of lucite which fit this hole and mounted to the chanenl with studs and wing nuts were provided. Black bakelite flow models were mounted directly to these small lucite sides.

The right cylinder had a diameter of 1/2-inch. The vertical throat separation of the orifice, venturi, and annular flow restrictor models totaled $5 / 8$ inch.

The orifice was $3 / 8$-inch wide and had a $45^{\circ}$ angle on the downstream side, sloping to a 1/16-inch wide throat. It was designed in this manner to give the necessary rigidity.

The venturi design was in accord with the published standards of the ASIME Committee on Fluid Meters.

The annular shape was based on the original NPR flow restrictor design. 


\section{RECIRCULATING LOOP EQUIPMENT}

The welded tank was of a 300 series stainless steel. All piping and fittings were 304 stainless steel, 3/4-inch nominal diameter, and Schedule 40 .

The pump was a centrifugal type, Number 106, of 316 stainless

steel. It is made by Easiern Industries and is driven by a $1 / 2 \mathrm{hp}$, 3450 rpm electric motor. It could produce a maximum flow in this facility of $14.5 \mathrm{gpm}$.

The initial globe valve was replaced early in the tests with a gate valve because plastic spheres readily became jammed. The gate valve was 18-8 stainless steel made by Chapman Valve Mfg. Co.

The flow meter was a size 6, 1700 series Fisher-Porter Flowrator Meter, with trim and float of $3 / 16$ stainless steel and calibrated as a laboratory standard.

\section{SUPER CENTRIFUGE}

To obtain the proper suspendion of bentonite clay, a high speed centrifuge was found to be indispensable. A laboratory-type Sharples Supercentrifuge driven by an air turbine was used. It is capable of speeds up to 50,000 rpm. The bentonite solutions were centrifuged between 43,000 and 48,000 rpm. It is shown in Figure XXI and a description of its use is found in Appendix F.

VISCOMETER

Original viscosity measurements were made using a standard Saybolt type instrument which measures time of discharge through a standard tube diameter. Because the bentonite is a non-newtonian fluid this method was totaliy unacceptable.

A rotational type viscometer was then used. The only instrument available was a RVF-100 Brookfield Synchro-lectric viscometer. In an attempt to minimize rheological influences, special cylindrical spindles were designed and used. This model is primarily intended for medium 
viscosity measurements ( 100 to $80,000 \mathrm{cps}$ ) and has four speeds. It is 15 years old, out of calibration, and the clutch tends to slip. Figure XXII shows the viscometer and cylindrical spindles.

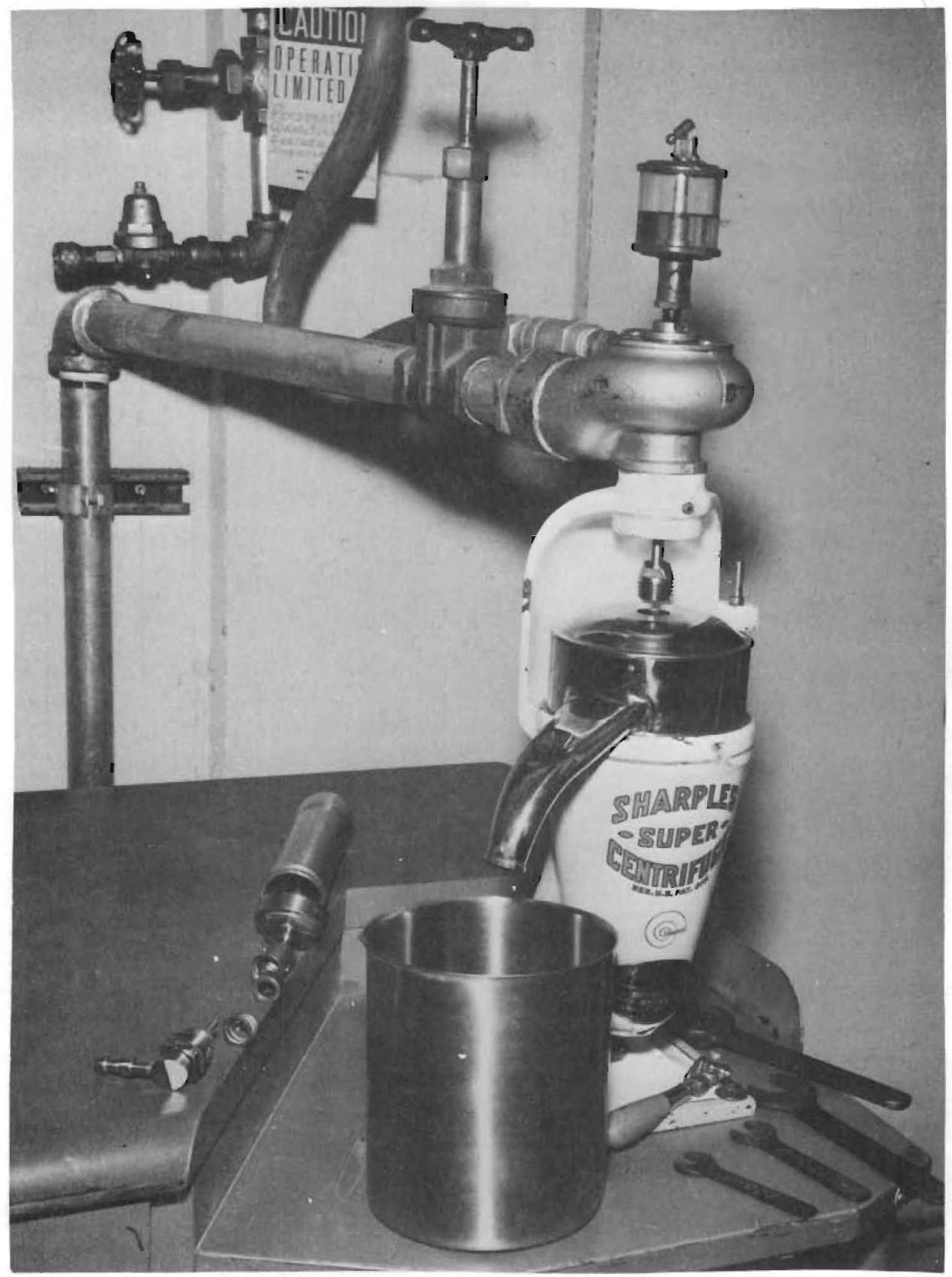

FIGURE XXI

Supercentrifuge 


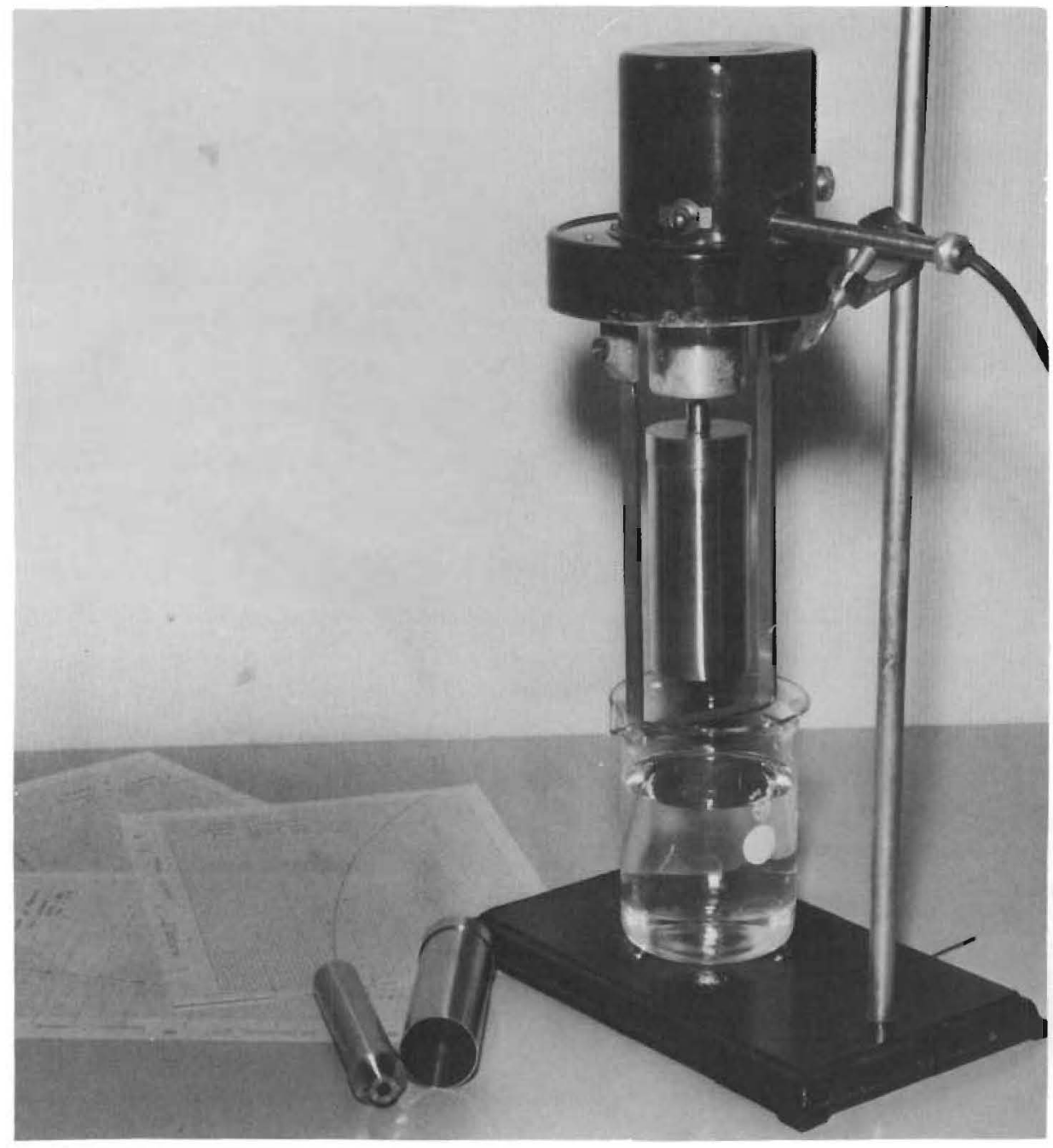

\section{FIGURE XXII}

Viscometer and Spindles

A Brookfield LVF model with a U. L. (ultra low viscosity) adapter is recommended for future use. 
APPENDIX E

BACKGROUND, THEORY, AND PROPERTIES OF SBR SOLUTIONS 


\section{APPENDIX E \\ BACKGROUND, THEORY, AND PROPERTIES \\ OF SBR SOLUTIONS}

\section{BACKGROUND OF USE}

The existence of the SBR phenomena was reported by Maxwell in 1873 when he described the action of Canadian Balsam when stirred. The solution was viewed through a polariscope. (24) A majority of the work on SBR fluids since that time has been by physical and colloidal chemists and concerned with the nature of the molecular and macromolecular action which causes streaming birefringence.

Humphrey ${ }^{(38)}$ demonstrated SBR using the colloid, vanadium pentoxide, in 1923, but did not utilize it nor analyze it.

In 1928, Ramon and Grishman ${ }^{(39)}$ and Kuhn ${ }^{(24)}$, proposed a theory of SBR for pure fluids. See following section on theory, this Appendix. Boeder developed a theory for colloids in 1932.

The first reported attempt at quantitative flow measurements was made in 1935 by Alcock and Sadron ${ }^{(40)}$ who used sesame oil. They based their results on the Ramon-Krishman theory. The birefringence was very small, a small fraction of a wave length, and had to be measured utilizing a spectroscopic technique associated with a compensator.

Early systematic attempts to use the SBR of colloidal solutions in flow studies were made by Hauser and Dewey $(41,42)$ in 1939 with a final report by Dewey ${ }^{(15)}$ in 1941. Early in World War II a polarized light flume was built by Knapp and his co-workers at the California Institute of Technology, based on personal advice from Dewey. It was used in design studies for underwater ordnance devices. Flow separation was readily seen on three-dimension models, allowing redesign of fairings, after body, and tail shapes. (43) 
Also based on Dewey's work, Leaf ${ }^{(27)}$ in 1945 reported on using a bentonite sol to analyze and successfuliy modify a steam locomotive firebox by installing a baffle to reduce localized cinder cutting. That same year, Binnie ${ }^{(44)}$ used a dye, benzopurpurin, in a study of the basic fluid mechanics phenomenon associated with the onset of turbulence in round channels. A photo-cell and cathode ray tube were used to detect the presence and magnitude of the turbulence. Further studies of a like nature were reported by Binnie and Fowler. ${ }^{(45)}$ They report problems with and questions concerning anamalous viscosities.

In 1947, Weller ${ }^{(46)}$ reported on work which had been done during World War II with a number of different organic liquids. All of the liquids found with good SBR properties had high viscosities. It is now known that these substances are non-Newtonian and exhibit visco-elastic behavior; i.e., they exhibit elastic recovery from deformations which occur during flow. In the study of two-dimensional hydro-dynamics of visco-elastic media, SBR appears to be the most powerful tool available. Phillippoff $(47,48)$, in work published in 1956 and 1959 , has demonstrated a particular visco-elastic theory, first stated by Lodge, and the procedure for studies of visco-elastic substances.

Jerrard $^{(49)}$ in 1950 and Wayland ${ }^{(50)}$ in 1955 discuss the onset of turbulence in the concentric cylinder device and conclude that rotation of the outer cylinder with the inner cylinder fixed is much more stable than rotation of the inner cylinder with a stationary outer. The concentric cylinder apparatus is basic for any quantjtative analysis work. Wayland studied both bentonite and a pure liquid, ethyl cinnamate.

Lindgren. ${ }^{(51)}$ in 1954 reported on the transition between laminar and turbulent flow in circular tubes, an extension of the work done by Binnie and Fowler. (45) He used a bentonite clay for his SBR fluid. The work by these men has been a contribution to understanding of this transition. 
In 1953, Pebbles and his associates ${ }^{(24)}$ reported on a dye, milling yellow, which has strong SBR characteristics. Pebbles, Prados, and Honeycutt from the University of Tennessee conducted an extensive study between 1954 and 1957 which was reported by Prados and Pebbles ${ }^{(52)}$ in 1959. They report that initial efforts were with bentonite clay solutions but that so many difficulties were experienced ir obtaining the proper grade of clay and in preparation that they investigated other substances. An attempt was made to use milling yellow during the investigations herein reported, but the technique used failed to produce any SBR. Previous investigators at Hanford also have had lack of success with milling yellow. Preparation of this solution appears to be as much of an art as does preparation of bentonite clays. Young and others ${ }^{(43)}$ have questioned some of Prados conclusions, particularly those related to rheological properties.

In $1962, \mathrm{Kemp}^{(2)}$ reported a very careful and complete calibration and analysis on bentorite sols. His work was an extension and improvement on work by Robinson in 1954. He discusses Boeder's theory in relationship to bentonite, in some depth, and states methods which could be used in quantitative analysis, but he does not analyze any flows. He concludes that the relationship between flow and SBR is more complicated than generally assumed. He also conciudes that SBR is related to the maximum rate of shear, Gm, and to the angle between the streamline and plane of action of $\mathrm{Gm}$. This result agrees with others. $(52,53,54)$

With three minor exceptions, the basic studies concerning the onset of turbulence ${ }^{(44,45,51)}$, Leal's design studies ${ }^{(27)}$, and a casual mention that turbulent flow is interesting ${ }^{(52)}$, all work concerning SBR and its relationship to fluid flow has been for flows in the laminar range. THEOR Y OF STREAMING BIREFRINGENCE

The theory of SBR at the present time is probably best summarized by Wayland ${ }^{(54)}$, who has made significant contributions to both the theoretical and experimental understanding of SBR phenomena, when he states, 
"the use of the SBR of a dilute colloidal solution for studying the character of a two-dimensional laminar flow has never been placed on a sound theoretical basis." A number of theories have been advanced which work fairly well for specific applications, but none appears to have gained general and wide-spread acceptance. It should be understood that the following list is by no means inclusive. It discusses only a few of the theories used for flow analysis. Jerrard ${ }^{(55)}$ gives an excellent review on over 23 theories.

The following list names some of these theories and gives further references:

1. Ramon-Krishman (1928) - Reference (39) and Kuhn (1932) Reference (24). This theory is for pure liquids, only, and states that the optic axis is parallel to the principal stress, or $45^{\circ}$ with the streamline. Considerable doubt about the validity of these theories ${ }^{(43)}$ had added support to the next theory.

2. Peterlin and Stuart - (1939) - Reference (43). This theory is also for pure fluids, only, and includes the effect of kinematic orientation of asymmetric molecules and certain internal field corrections due to molecular interactions.

3. Lodge (1955) - Reference (43). This theory applies to viscoelastic liquids. He predicted that the stress ellipsoid and the refractive index ellipsoid have the same orientation, their asymmetries are proportional to one another, and that there is a tensile stress in shear flow in the direction of the streamlines. There is experimental support for this theory.

4. Boeder (1932) - Reference (56) and Kuhn (1932) - Reference (56) and (24). Boeder attributed SBR to the kinematic orientation of anisometric particles under the combined action of hydrodynamic forces and Brownian movement. To approximate a rod, a dumb-bell shape is assumed. Rosenburg ${ }^{(53)}$ extends this theory and Kemp ${ }^{(56)}$ discusses Boeder ${ }^{3}$ s theory in some detail. 
5. Wayland (1960) - Reference (54) and (16). His theoretical treatment is for rigid ellipsoids of revolution in general, twodimensional, laminar flow and includes equilibrium between hydrodynamic force from flow and Brownian motion. It neglects hydrodynamic and chemical interactions between particles.

\section{PROPERTIES OF BENTONITE SOLS}

The use of the term "bentonite" is perhaps not rigorous enough because Kemp ${ }^{(56)}$ has shown that not all types of this clay will produce the desired SBR. Most of the following is from Kemp's study.

Bentonite is a name given to a group of naturally occurring clays, probably of volcanic origin, found in South Dakota, Wyoming, Arkansas, and California. It gets its name from Fort Benton, Montana, where it was originally mined.

The principle constituent is Montmorillonite, a mineralogical term, describing essentially hydrated silicates of aluminum. Montmorillonite has an unusually large base exchange capacity which permits replacement of aluminum by potassium, magnesium sodium, or calcium.

Electron microphotographs show the individual particle shapes of bentonite to range from a fluffy amorphous - appearing material to thin plates with a variable diameter-to-thickness ratio from $5: 1$ to $50: 1$. Unit plates were observed 9 angstroms thick.

However, clays mined near Hector, California are magnesium rich and electron microphotographs of these reveal lath shaped particles rather than the plate shape. These lath shaped particles are typical of the trioctahedral montmorillonites, a fact which is not predictable from theory. These particles have a length-to-width ratio of about $10: 1$. Lengths varied from 5000 to 10,000 angstroms, thickness variation was from about 10 to 20 angstroms. 
Any suspension of asymmetrical particles will become birefringent if (1) a preferred orientation is imposed on them, and (2) if their refractive index differs from the fluid or if the particles themselves are anisotropic。

It has been shown that the intrinsic birefringence of the Hectorite particles is sufficient to account for the observed SBR. There is also evidence to suggest that differences in Montmorillonite structure result in differences in SBR.

Kemp reports a variation between two different deliveries of the Hectorite powder. The only noted difference between the two was a detail in the grinding procedure, with possible localized overheating.

The majority of problems associated with handling and using bentonites arise from the rheopetic properties of colloids. Generally, these fall in three classes: Flocculation, Gelation, and Anomalous Viscosities.

Flocculation. Flocculation occurs when particles collide and adhere due to Van der Waals forces. These particles then settle out.

Bentonite particles have been shown to possess an "electrical double layer." This electrical double layer is formed when the particle surface becomes negatively charged due to preferential adsorption of $\mathrm{OH}$ ions or to the disassociation of surface cations. This results in a diffuse layer of oppositely charged ions around the particle which are referred to as counter ions. The Van der Waals force is opposed by this electrical double layer force. When the kinetic energy is sufficient to overcome the electrical potential energy, flocculation will commence.

Gelation. Bentonites and certain other hydrophylic suspensions can be transformed from a liquid to a gel. If a mechanical action causes a return to the liquid state, this is known as a thixotropic material. When the liquid-to-gel action is accelerated by a gentle mechanical motion this phenomena is called rheopexy. There are three theories of 
gelation, all of which have received certain objections. They all concern electrical double layers and other forces - such as Van der Waals forces and are thus closely related to flocculation.

Peptization. The addition of a small quantity of electrolyte will raise the charge on the particle due to the increased adsorption of ions. Thus, the dispersion of the particles will be increased, the maximum potential energy increased, and the solution less likely to flocculate. This peptizing effect is stronger when polyvalent ions are involved. By analogy, it seems reasonable to believe that the proper amount of peptizing agent will reduce any tendency to rheopectic gelation. Too much electrolyte, on the other hand, will cause the solution to gel or flocculate.

Anomalous Viscosity. When the coefficient of viscosity is a function of the rate of shear, the fluid is called non-Newtonian and is often referred to as having an anomalous viscosity.

Most colloidal suspensions, bentonites included, fall into this classification. The rate of energy dissipation from the suspended particles; i.e., viscosity, is a function of particle orientation. At low flows, where shear forces are about the same as Brownian forces, the dissipation of energy is maximum. In the preferred orientation of faster flows this energy dissipation is minimum. The apparent viscosity is, therefore, correspondingly maximum at low flows and minimum at high flows.

The result of Kemp's measurements on four concentrations is given in Figure XXIII. These solutions contained 0.06 grams of sodium pyrophosphate peptizing agent per gram of Hectorite. Concentrations used in the comparative study of this report varied from 0.342 to 0.743 .

The first volume of bentonite fluid mixed for our tests was useable for a period of 2-1/2 months. At the end of 3-1/2 months the solution had gelled to the point that it would not flow from a horizontal beaker. The peptizing agent, sodium pyrophosphate, was added to the second and third batches. These batches were noticeably more stable and less prone to gel and are still useable after 4 months. This effect was observed on 
samples located on contaminated test plates, within the flow channels and when stored in their plastic storage bottles.

For a more detailed discussion involving measurements on nonNewtonian fluids see Schowalter's ${ }^{(57)}$ recent article.

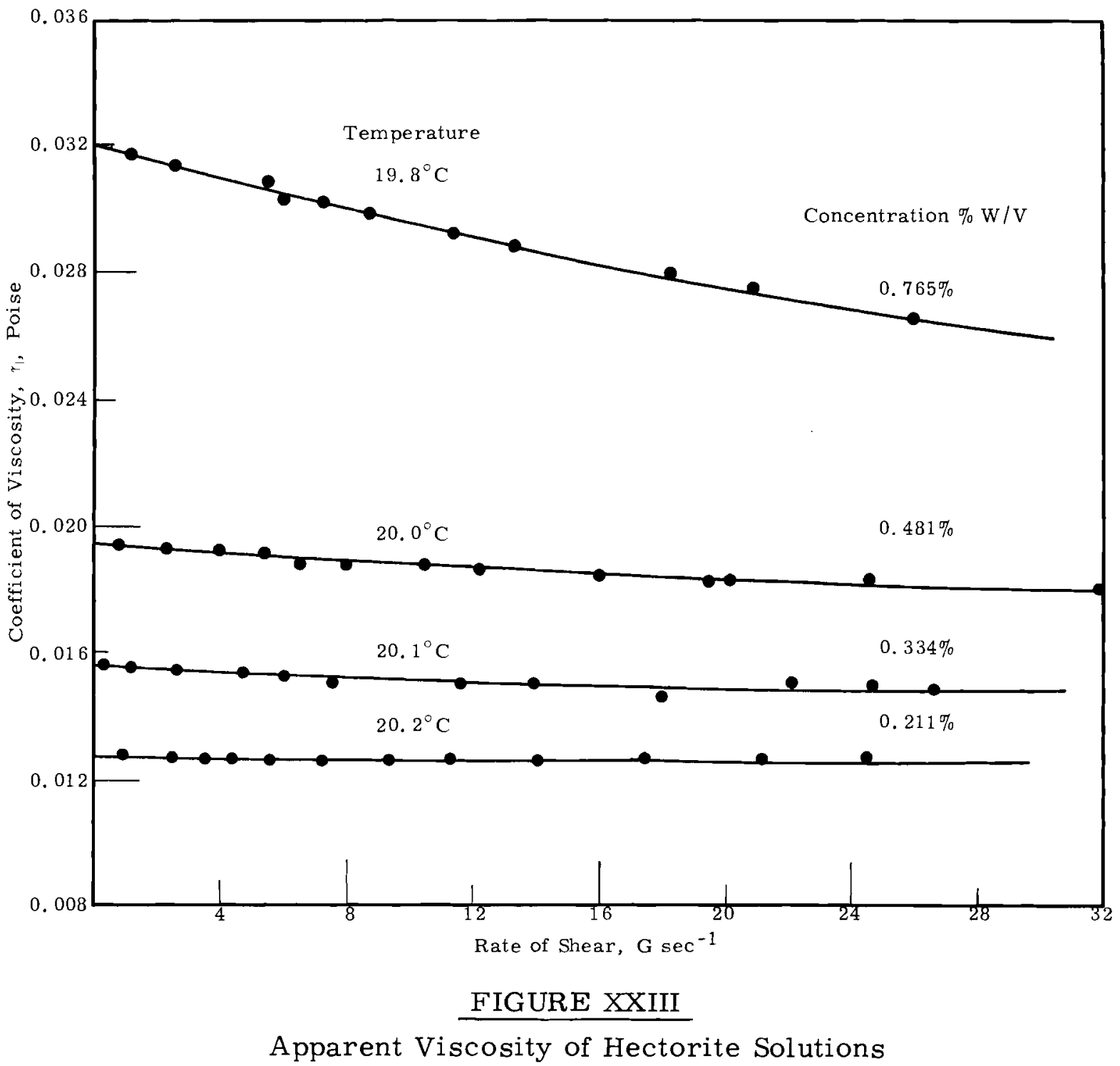




\section{APPENDIX F}

PREPARATION AND HANDLING OF BENTONITE SOLUTIONS 


\section{APPENDIX F}

\section{PREPARATION AND HANDLING \\ OF BENTONITE SOLUTIONS}

\section{PREPARATION}

The clay used in preparing useable SBR fluids was a "Spray Dried Hector Clay" obtained from the National Lead Company, St. Louis. Kemp ${ }^{(56)}$ reports that Hectorite is the best available clay of the bentonite family.

Acceptable solutions could not be obtained using the method of preparation reported by Leaf. (27) Using preparation procedures reported by Kemp ${ }^{(56)}$ gave fluids of only marginal SBR properties. In addition, Kemp recommends settling of the solution over-night to remove large particles and centrifuging at 25,000 rpm. Quiet storage of our solutions for 30 hours and very careful syphoning from the top showed no settling. Centrifuge speeds under 20,000 rpm showed no appreciable difference between uncentrifuged samples.

This leads to the speculation that minor differences in the clay can make significant differences in the SBR solution. Kemp ${ }^{(56)}$ reported excessive milling had rendered one batch of his clay unuseable.

It can only be concluded that preparation of useable Hectorite SBR solutions is possible, but borders on the "artistic".

The method of preparation used for these was:

1. Measure 15 to 25 liters of deionized water into a polyethylene mix tank.

2. Add 16 grams of Hectorite per $1000 \mathrm{cc}$ of water by sprinkling the clay lightly on the water surface. Each small sprinkling was thoroughly mixed using an air driven, three-inch diameter, stainless steel, propeller-type mixer. Complete mixing was accomplished before the next "sprinkling". 
3. Add 0.06 gram per gram of Hectorite of the tetravalent salt, sodium pyrophosphate $\left(\mathrm{Na}_{4} \mathrm{P}_{2} \mathrm{O}_{7} \cdot 10 \mathrm{H}_{2} \mathrm{O}\right)$ as a peptizing agent.

4. Agitate the solution for 4 to 6 hours and allow to settle from 17 to 30 hours.

5. Syphon the solution into a plastic bottle. This bottle was on a hand hoist so it could be raised to allow gravity feed to the centrifuge.

6. First, centrifuge the solution through a nozzle with a 0.100 inch diameter opening, and second, through a nozzle with a 0.045 inch diameter opening.

The smaller opening gave better SBR characteristics but tended to clog. It was found that time could be saved by two passes through the centrifuge rather than frequent cleaning of the smaller nozzle.

A great deal of trouble was experienced with leaking from the continuous feed centrifuge. An estimated $70 \%$ of the runs had to be stopped, the apparatus cleaned and reassembled, and restarted due to leakage. The cause of the leakage is unknown.

$\underline{\text { HANDLING }}$

The SBR Hectorite solution was stored in polyethylene carboys when not in use. Solution was allowed to stand in the stainless steel tank for up to three days. Excessive gelation on and around the inlet pipe took place if left longer.

Rapid gelation was experienced on test samples contaminated with dust, small particles of saw dust, and carbon steel. The bentonite caused rapid corrosion of the carbon steel.

Shearing of the fluid in the viscometer or from use in the flow facility did not appear to increase the tendency to gel as has been reported by Kemp. (56)

Thus, in summary, once the technique for preparing useable solutions was established only minor problems were experienced with mixing and handling of the SBR Hectorite solution. 


\author{
APPENDIX G \\ PREPARATION AND HANDLING \\ OF OTHER VISUALIZATION TECHNIQUES
}




\section{$\underline{\text { APPENDIX G }}$ \\ PREPARATION AND HANDLING \\ OF OTHER VISUALIZATION TECHNIQUES}

This appendix briefly outlines the preparation and handling of tracers, tufts, and dye injection used in these tests.

\section{TRACERS}

The first tracers tried were sawdust particles. A series of different mixtures and different "sifting" screen sizes were investigated in an attempt to attain neutral particle suspension.

When virgin fir sawdust particles were added to a beaker of water, mixed, screened, and allowed to stand, some would sink, some would float, and a few would slowly settle. Techniques which separated the heavy and light particles from those slowly settling ones produced a solution containing fairly neutral density tracers. This solution gave patterns which were visible to the eye, but which could not be photographed very successfully. Techniques using fluorescent dyes to color the sawdust and ultraviolet illumination to make it visible also were unsuccessful.

Small polystyrene sphere were obtained and mixed in a ratio of $3 \mathrm{cc}$ of beads to $1000 \mathrm{cc}$ of water. Their specific gravity ranged from 1.02 to 1.05 . Diameters ranged from 0.026 to 0.052 inch with a majority between 0.031 and 0.036 inch.

Being spherical and transparent made them efficient light reflectors and they photographed well. However, they settled out before reaching the models and tended to clog the control valve at low flows.

The globe valve was replaced with a gate valve which helped reduce the clogging somewhat.

Glycerol was then added to the tank and the fluid circulated at a low velocity. The glycerol-water-plastic sphere mixture had a specific gravity of 1.025 and a viscosity of 1.4 centipoise. Even using this 
method, some spheres floated, some sank and some adhered together in large batches at the fluid surface in the tank. Temperature changes of 5 to 10 degrees visibly affected the number of suspended particles.

Beads were easily removed by filtering the exit flow through a cloth. Thus, beads could readily be added or removed at will from the system.

TUFTS

The tufts were pieces of cotton thread approximately 0.008 inch in diameter. Their lengths varied between $1 / 8$ to $1-1 / 4$ inch, with the majority near $1 / 2$ inch.

The bakelite models were marked along the centerline and small holes made in the surface by hand using a sharp scribe. The thread was cut to length, held in place with tweezers, and fixed to the plastic with a drop of plastic solvent from the tip of a pencil.

These tufts were too stiff for the flows of this test and had too many hairlike fuzz threads which trapped air bubbles. DYE

The dye used was an aqueous solution of potassium permanganate $\left(\mathrm{K} \mathrm{MnO}_{4}\right.$ ). Approximately $1 / 2 \mathrm{cc}$ of $\mathrm{K} \mathrm{MnO}_{4}$ crystals were added to $50 \mathrm{cc}$ of deionized water.

Two holes, 0.031 inch diameter, were drilled in the top of the channel up-stream from the models.

A hypodermic needle, 0.0285 inch OD and 1-1/2 inch long, was inserted in one hole and approximately $1 / 8$ inch of the tip was bent at right angles. The other hole was plugged. A $10 \mathrm{cc}$ hypodermic syringe was filled with dye and attached to the needle.

Dye was injected at a steady rate by placing very small weights on the syringe plunger. Dye entered the fluid stream parallel to the mean flow and the vertical position was maintained manually. 
Starting with 175 liters of clear water up to $50 \mathrm{cc}$ of dye could be added without adverse clouding of the fluid. 
APPENDIX H

ANALYSIS TECHNIQUES

OF LAMINAR FLOWS UTILIZING SBR PROPERTIES 


\section{APPENDIX H \\ ANALYSIS TECHNIQUES \\ OF LAMINAR FLOWS UTILIZING SBR PROPERTIES}

It has been demonstrated that the SBR patterns resulting from steady flow in the laminar range do not vary with respect to time. If the relationship which exists between these patterns and the parameters which define a flow system can be determined, it is apparent that flows can be quantitatively analyzed by using SBR fluids.

A two dimensional flow state is completely determined if the streamline directions and the point velocities are known. It is recalled from Appendix B that the SBR isochromatic fringes are functions of strain rates, or stresses, and the isoclinic fringes are functions of the direction of these strain rates.

An early attempt to establish a workable relationship between the required streamline directions and velocities and the measured fringe patterns was done by Dewey in 1941. He assumed the same relationship between SBR and fluid stresses as exists between stress and photoelasticity. More recently, Pracios ${ }^{(14)}$, Sutera and Wayland ${ }^{(16)}$, and Kemp ${ }^{(56)}$ have shown that techniques based on a strict analogy between SBR and photoelastic analysis was a simplification true only for certain cases of parallel, pure shear flow.

The resulting relationships determined by these investigators are complex and based on rather restrictive assumptions. The most complicated analysis found was for laminar flow around a right circular cylinder at a Reynolds number of $3080 .^{(14)}$ This analysis was long and involved.

The purpose of this appendix is to present an outline of these analysis methods. One method will be presented in some detail and the results and conclusions, only, for the other two. 
PRADOS ANALYSIS FOR TWO-DIMENSIONAL LAMINAR FLOW

Prados assumed simple relationships between the birefringence and rate of strain and between the isoclinic axis and the direction of the strain rate. These relationships were developed from empirical tests with milling yellow, an organic dye.

He also assumed a continuum fluid with a constant viscosity. Consider a square element of such a continuum, oriented parallel to a system of cartesian coordinates as in Figure XXIV.

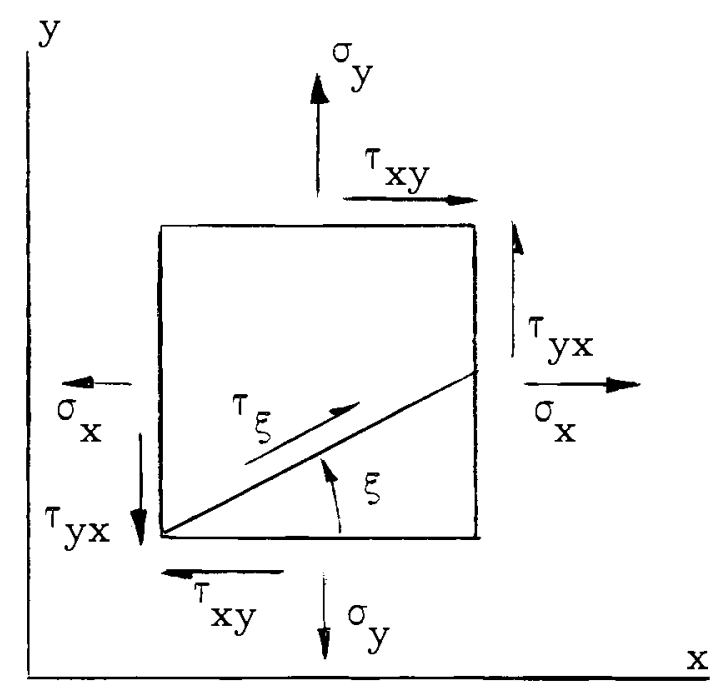

\section{FIGURE XXIV}

Elemental Stress Relationships

Balancing forces shown in the figure gives:

$$
\tau_{\xi}=\frac{\sigma_{y}-\sigma_{x}}{2} \sin 2 \xi+\tau_{x y} \cos 2 \xi
$$

The stresses, $\sigma_{\mathrm{x}}, \sigma_{\mathrm{y}}$, and $\tau_{\mathrm{xy}}$ are expressed in terms of velocity gradients from the Navier--Stokes development. The results for incompressible two-dimensional flow, as given by Streeter are: 


$$
\begin{aligned}
\sigma_{x} & =-P-2 \mu \frac{\partial u}{\partial x} \\
\sigma_{y} & =-P-2 \mu \frac{\partial v}{\partial y} \\
\tau & =\mu\left(\frac{\partial u}{\partial y}+\frac{\partial v}{\partial x}\right)
\end{aligned}
$$

where:

$$
\begin{aligned}
& P=\text { hydrostatic pressure } \\
& u=\text { viscosity } \\
& u=\text { velocity in } x \text { direction } \\
& v=\text { velocity in } y \text { direction }
\end{aligned}
$$

Substitution gives:

$$
\tau_{\xi}=\mu\left(\frac{\partial v}{\partial y}-\frac{\partial u}{\partial x}\right) \sin 2 \xi+\mu\left(\frac{\partial u}{\partial y}+\frac{\partial v}{\partial x}\right) \cos 2 \xi
$$

Since

$$
\tau_{\xi}=\mu \mathrm{E}_{\xi}
$$

where

$$
\mathrm{E}_{\xi}=\text { rate of deformation along plane of action at angle } \xi
$$

It is desirable to transform equation (1) from Cartesian to Stream coordinates. Referring to Figure XXI the required transformations are:

$$
\begin{aligned}
\Delta \mathrm{s} & =\Delta \mathrm{x} \cos \varphi+\Delta \mathrm{y} \sin \varphi \\
\Delta \mathrm{n} & =-\Delta \mathrm{x} \sin \varphi+\Delta \mathrm{y} \cos \varphi \\
\mathrm{u} & =\mathrm{V} \cos \varphi \\
\mathrm{v} & =\mathrm{V} \sin \varphi
\end{aligned}
$$

where:

$$
\begin{aligned}
& \mathrm{s}=\text { distance along streamline } \\
& \mathrm{n}=\text { distance along curve normal to streamlines } \\
& \mathrm{V}=\text { velocity along streamline }
\end{aligned}
$$




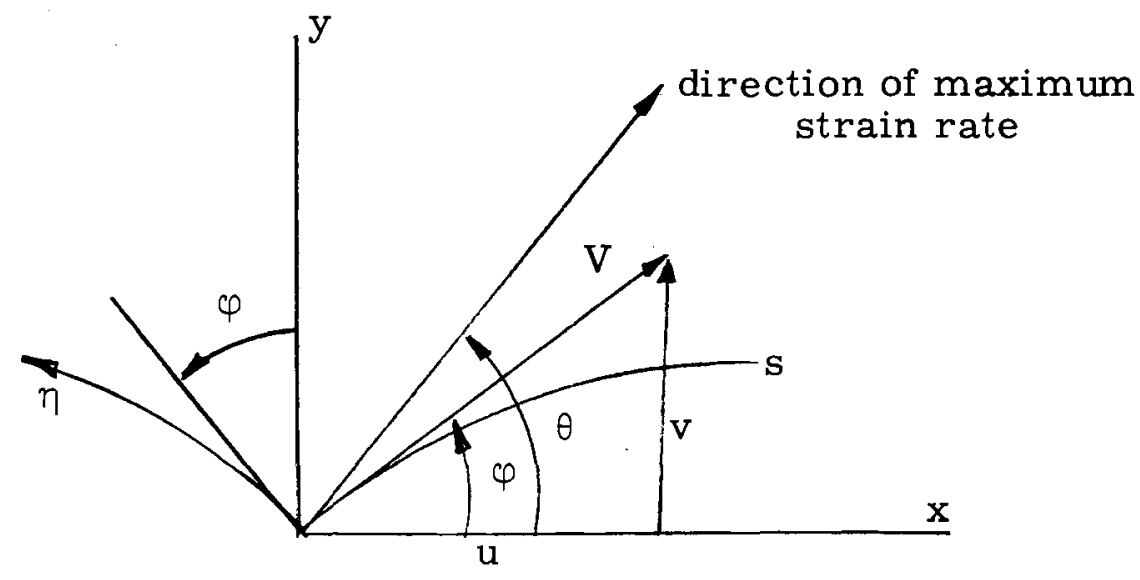

\section{FIGURE XXV}

Transformation Relationships

note that

$$
\begin{aligned}
& \Delta s=s(x, y) \\
& \Delta n=n(x, y)
\end{aligned}
$$

and choose

$$
f=f(x, y)
$$

then:

$$
\begin{aligned}
& d f=\frac{\partial f}{\partial s} d s+\frac{\partial f}{\partial n} d n \\
& \frac{d f}{d x}=\frac{\partial f}{\partial s} \frac{d s}{d x}+\frac{\partial f}{\partial n} \frac{d n}{d x} \\
& \frac{d f}{d y}=\frac{\partial f}{\partial s} \frac{d s}{d y}+\frac{\partial f}{\partial n} \frac{d n}{d y}
\end{aligned}
$$

Evaluating these from the transformation gives:

$$
\begin{aligned}
& \frac{\partial f}{\partial x}=\cos \varphi \frac{\partial f}{\partial s}-\sin \varphi \frac{\partial f}{\partial n} \\
& \frac{\partial f}{\partial y}=\sin \varphi \frac{\partial f}{\partial s}+\cos \varphi \frac{\partial f}{\partial n}
\end{aligned}
$$


Substituting into the desired velocity components gives:

$$
\begin{aligned}
\frac{\partial v}{\partial y}-\frac{\partial u}{\partial x} & =\frac{\partial}{\partial y}(V \sin \varphi)-\frac{\partial}{\partial x}(V \cos \varphi) \\
& =V \cos \varphi \frac{\partial \varphi}{\partial y}+\sin \varphi \frac{\partial V}{\partial y}+V \sin \varphi \frac{\partial \varphi}{\partial x}-\cos \varphi \frac{\partial V}{\partial x}
\end{aligned}
$$

Letting $V=f$, and then $\varphi=f$ :

$$
\begin{aligned}
\frac{\partial \mathrm{V}}{\partial \mathrm{y}}-\frac{\partial \mathrm{u}}{\partial \mathrm{x}}= & V \cos \varphi\left(\sin \varphi \frac{\partial \varphi}{\partial s}+\cos \varphi \frac{\partial \varphi}{\partial \mathrm{n}}\right) \\
& +\sin \varphi\left(\sin \varphi \frac{\partial V}{\partial s}+\cos \varphi \frac{\partial V}{\partial \mathrm{n}}\right) \\
& +V \sin \varphi\left(\cos \varphi \frac{\partial \varphi}{\partial s}-\sin \varphi \frac{\partial \varphi}{\partial \mathrm{n}}\right) \\
& +\cos \varphi\left(\cos \varphi \frac{\partial V}{\partial s}-\sin \varphi \frac{\partial V}{\partial \mathrm{n}}\right)
\end{aligned}
$$

Collecting terms and simplifying:

$$
\frac{\partial V}{\partial y}-\frac{\partial u}{\partial x}=\sin 2 \varphi\left(\frac{\partial V}{\partial n}+V \frac{\partial \varphi}{\partial s}\right)-\cos 2 \varphi\left(\frac{\partial V}{\partial s}-V \frac{\partial \varphi}{d n}\right)
$$

In a like manner

$$
\frac{\partial u}{\partial y}+\frac{\partial V}{\partial x}=\cos 2 \varphi\left(\frac{\partial V}{\partial n}+V \frac{\partial \varphi}{\partial s}\right)+\sin 2 \varphi\left(\frac{\partial V}{\partial s}-V \frac{\partial \varphi}{\partial n}\right)
$$

Combining Equations (1), (2), (3), (4) gives:

$$
\begin{aligned}
E_{\xi}= & -\left[\left(\frac{\partial V}{\partial s}-\frac{\partial \varphi}{\partial n}\right) \cos 2 \varphi-\left(\frac{\partial V}{\partial n}+V \frac{\partial \varphi}{\partial s}\right) \sin 2 \varphi\right] \sin 2 \xi \\
& +\left[\left(\frac{\partial V}{\partial n}+V \frac{\partial \varphi}{\partial s}\right) \cos 2 \varphi+\left(\frac{\partial V}{\partial s}-V \frac{\partial \varphi}{\partial n}\right) \sin 2 \varphi\right] \cos 2 \xi
\end{aligned}
$$

Simplifying, and noting that

$$
\frac{\partial \varphi}{\partial s}=\frac{1}{r} \quad \text { and } \quad \frac{\partial \varphi}{\partial n}=\frac{1}{r^{\prime}}
$$

where:

$$
\begin{aligned}
r & =\text { radius of curvature of the streamline } \\
r^{\prime} & =\text { radius of curvature of the streamline normal }
\end{aligned}
$$


gives:

$$
\mathrm{E}_{\xi}=\left(\frac{\partial \mathrm{V}}{\partial \mathrm{n}}-\frac{\mathrm{V}}{\mathrm{r}}\right) \cos (2 \varphi-2 \xi)+\left(\frac{\partial \mathrm{V}}{\partial \mathrm{s}}-\frac{\mathrm{V}}{\mathrm{r}^{\prime}}\right) \sin (2 \varphi-2 \xi)
$$

Note that the rate of pure shear strain, G, in stream coordinates is:

$$
\mathrm{G}=\frac{\partial \mathrm{V}}{\partial \mathrm{n}}-\frac{\mathrm{V}}{\mathrm{r}}
$$

and the dilation rate, $\mathrm{D}$, is given by:

$$
\mathrm{D}=\frac{\partial \mathrm{V}}{\partial \mathrm{S}}-\frac{\mathrm{V}}{\mathrm{r}^{\prime}}
$$

Substituting these into Equation (5) gives

$$
\mathrm{E}_{\xi}=\mathrm{G} \cos (2 \varphi-2 \xi)+\mathrm{D} \sin (2 \varphi-2 \xi)
$$

Since SBR yields information about the maximum strain rate, $\mathrm{E}$, Equation (6) is differentiated and set equal to zero; i. e.:

$$
\frac{\mathrm{d} E}{\mathrm{~d} \xi}=2 \mathrm{G} \sin (2 \varphi-2 \varepsilon)-2 \mathrm{D} \cos (2 \varphi-2 \xi)
$$

set $\frac{d E}{d \xi}=0$ and note that $\varepsilon=\theta$ by definition, to get:

$$
\begin{aligned}
& \tan (2 \varphi-2 \theta)=\frac{D}{G} \\
& \sin (2 \varphi-2 \theta)=\frac{D}{\left(D^{2}+G^{2}\right)^{1 / 2}}
\end{aligned}
$$

and

$$
\cos (20-2 \theta)=\frac{G}{\left(D^{2}+G^{2}\right)^{1 / 2}}
$$

and substituting into Equation (6) yields

$$
E=\sqrt{G^{2}+D^{2}}
$$

since

$$
\frac{\partial V}{\partial S}+\frac{V}{r}=0
$$


from the continuity equation in stream coordinates, the dilation rate becomes

$$
D=\frac{-2 V}{r^{1}}
$$

and

$$
\mathrm{D}^{2}=\frac{4 \mathrm{~V}^{2}}{\left(\mathrm{r}^{\prime}\right)^{2}}
$$

substituting again gives

$$
E=\sqrt{\left(\frac{\partial V}{\partial n}-\frac{V}{r}\right)^{2}+\frac{4 V^{2}}{\left(r^{\prime}\right)^{2}}}
$$

or re-arranging:

$$
\frac{\partial V}{\partial n}=\frac{V}{r} \pm \sqrt{E^{2}-\frac{4 V^{2}}{\left(r^{\prime}\right)^{2}}}
$$

This equation relates a velocity gradient to strain rate. Velocity is what we desire and strain rate information is found from SBR measurements. Note, however, that the radius to the streamline normal, $r^{\prime}$, is required to solve this equation. Or, saying it another way, the streamline pattern must be known before the velocity is computed.

Thus, it is seen that the angle between the maximum strain rate and the streamline is desired. Referring to Figure XXI this angle is $\theta-\omega$ which shall be called $\beta$.

Noting that Equation (7) can be rewritten as

$$
2 \varphi-2 \theta=\arctan \frac{D}{G}
$$

and substituting gives,

$$
\begin{aligned}
\theta-\varphi & =\beta=-1 / 2 \arctan \frac{D}{G} \\
\beta & =-1 / 2 \arctan \left(\frac{2 V}{r^{\top}} \frac{\partial n}{\partial V}-\frac{2 r}{r^{\top}}\right)
\end{aligned}
$$


Note that $\beta=0$; i. e., the line of maximum strain rate coincides with the streamline, only when the dilation rate, D, equals 0 . This occurs, of course, when the streamlines are parallel.

Thus, these equations are useful only when, (1) the streamline pattern is known from either boundary conditions or from some other method, not related to SBR, or (2) when it is known that no divergence or convergence exists and streamline patterns can be obtained from isoclinic measurements.

EXAMPLES OF SPECIFIC CASES

\section{Case I}

For flows with straight parallel streamlines, Equation (8) reduces to:

$$
\frac{\partial V}{\partial n}= \pm E
$$

since $\frac{\mathrm{V}}{\mathrm{r}}$ and $\frac{\mathrm{V}}{\mathrm{r}^{\mathrm{r}}}$, vanish.

Actual analysis involves the measurements of isochromatic fringes along a vertical line from the bottom to the top of the channel. The strain rate associated with each isochromatic is obtained from the optical calibration curves. Since boundary conditions are easily obtained from the channel edges, it is a simple matter to integrate graphically the curve of deformation rate versus distance to obtain the curve of velocity versus distance. Note that the streamline directions are known for this simplifying case.

\section{Case II}

For flows with parallel, but curved streamlines (such as is found in the concentric cylinder apparatus commonly used for SBR calibrations) the Equation (8) reduces to:

$$
\frac{\partial V}{\partial n}= \pm E+\frac{V}{r}
$$


since

$$
\frac{V}{r}=0
$$

and

$$
\theta=\varphi .
$$

The analysis method is similar to Case I except the radius of curvature of the streamlines is required. These can be obtained from non-SBR sources by either graphical or numerical means. Once again the streamlines must be knowr.

For a discussion of more complicated flows, References (14) and (52) are recommended。

\section{LIMITATIONS}

It must be remembered that these equations were based on the assumption that the test fluid conforms to the restrictions of the Stokes law of friction; namely, a viscous continuum with a constant viscosity.

The phenomenon of birefringence is possible only because of the anisotropic nature of certain kinds of matter. In the case of a bentonite clay suspension, and other SBR fluids, rheopetic and anomalous prop-

erties have been frequently observed. Prados and Pebbles ${ }^{(52)}$ report Newtonian behavior of their SBR fluid at low strain rates and used this observation in the preceding analysis. Since the very nature of the fluid which accounts for its SBR properties also causes anomalous variations in Newtonian fluid parameters, one must judge carefully those cases where this simplifying assumption is valid.

This analysis also assumed the following two relationships: (1) that the amount of birefringence is a single-valued, increasing function of the rate of strain and (2) that the isoclinic axes bear a simple relationship to the direction of maximum shear stress in the liquid. These were based on empirical tests with a concentric cylinder apparatus. Kemp ${ }^{(56)}$ and Wayland ${ }^{(54)}$ have considered the effects of these in their analysis. A statement of their results and conclusions follows: 


\section{SUMMARY OF KEMP'S INVESTIGATION}

Kemp based his analysis on Boeder's theory of birefringence which assumes dumbbell shaped particles influenced by hydrodynamic and Brownian type forces. He derives an expression for the rotation of a particle in non-parallel, steady-state flow of the form:

$$
\frac{\partial^{2} F}{\partial \theta^{2}}-\frac{\partial F}{\partial \theta} \frac{E}{D}\left(\sin ^{2} \theta-\sin ^{2} \beta\right)-\frac{F E}{D} \sin 2 \theta=0
$$

where:

$$
\begin{aligned}
F= & \text { density of orientation function defined by } \\
F= & \lim _{\Delta \theta \rightarrow 0} \frac{\Delta N}{\Delta \theta} \\
\Delta \mathrm{N}= & \text { number of particles per unit volume whose axis lie } \\
& \text { between } \theta \text { and } \theta+\Delta \theta . \\
\theta= & \text { angle between the particle and the maximum strain } \\
D= & \text { rotational diffusion coefficient, the magnitude of } \\
& \text { depends on particle size, particle shape, solution } \\
& \text { viscosity, and solution temperature. } \\
\mathbf{E}= & \text { maximum strain rate }
\end{aligned}
$$

His relationship shows that orientation of the particles is a function of rotation and maximum strain rates. He investigates a number of values of $\frac{E}{D}$ and of $B$ and concludes the following:

1. That SBR of colloidal suspensions depends on $\mathbf{E}$ and $B$.

2. Calibrations curves obtained from a concentric cylinder apparatus are only approximately applicable to non-parallel flows. When $\beta<10$ degrees this approximation is very close.

3. In non-parallel flows with small values of $\beta$, the magnitude and plane of action of $\mathrm{E}$ can be found directly from the calibration curves. 


\section{SUMMARY OF WAYLAND'S THEORETICAL ANALYSIS}

Wayland has considered the orientation of macromolecules in dilute solutions and compared the results with SBR measurements in an eccentric cylinder apparatus. His analysis takes into account hydrodynamic forces and Brownian motions, assumes that the reaction time of the particles is short compared to the time required to transport the particles to a different hydrodynamic environment, and neglects hydrodynamic and chemical interactions between particles.

The theoretical results, which assumed rigid, ellipsoidal particles, are:

$$
\theta=\frac{E \sin 2 \Lambda o}{6 D_{1}}\left[1-\frac{E^{2}}{27 D^{2}} \sin ^{2} 2 \Lambda 0+\frac{24 b^{2}}{35}+\cdots\right]
$$

where

$\theta=$ angle between the maximum strain rate axis and the optic axis.

$\mathbf{E}=$ maximum strain rate

$\Lambda 0=$ angle between streamline and maximum strain rate

$$
\mathrm{b}=\frac{\mathrm{a}_{1}^{2}-\mathrm{a}_{2}^{2}}{\mathrm{a}_{1}^{2}+\mathrm{a}_{2}^{2}}
$$

$a_{1}=$ semimajor axis of ellipsoidal particle

$a_{2}=$ semiminor axis of ellipsoidal particle

$\mathrm{D}=$ rotary diffusion coefficient

$$
=\frac{k \operatorname{Tr}^{2}}{\eta \circ V r\left(r^{2}-1\right)}\left[\frac{2 r^{2}-1}{r\left(r^{2}-1\right)} \ln \left\{r+\left(r^{2}-1\right)^{1 / 2}\right\}-1\right]
$$

$\mathrm{k}=$ Boltzmann's constant

$r=\frac{a_{1}}{a_{2}}=$ axial ratio 


$$
\begin{aligned}
T & =\text { temperature, absolute } \\
V & =\text { volume of the particle } \\
\eta_{O} & =\text { viscosity felt by the particle }
\end{aligned}
$$

and:

$$
\Delta \eta=\frac{4 \pi \mathrm{cGEb}}{15 \eta \bar{D}} 1-\frac{\mathrm{E}^{2}}{18 \mathrm{D}^{2}} \sin ^{2} 2 \Lambda \mathrm{o}+\frac{6 \mathrm{~b}^{2}}{35}+\ldots
$$

where:

$$
\begin{aligned}
& c=\text { volume concentration of ellipsoids } \\
& \eta=\text { mean index of refraction of the solution } \\
& G=\text { optical anisotropy of the particles }
\end{aligned}
$$

Note that in flows where both $\theta$ and $\Delta \eta$ are linear with strain rate, these equations reduce to:

$$
\begin{aligned}
\theta & =c_{1} E \sin 2 \Lambda O \\
\Delta \eta & =c_{2} E
\end{aligned}
$$

Where the constants, $c_{1}$, and $c_{2}$, are experimentally evaluated for a given fluid.

Sutera $^{(16)}$, for his Ph. D dissertation, ran a series of tests on a birefringent fluid to check these results. It was concluded that the relationships between SBR and the hydrodynamic parameters can be theoretically predicted as long as the fluid is a very dilute solution with rigid colloidal particles whose relaxation time is short compared to its rate of change of the hydrodynamic environment.

$\underline{\text { REMARKS }}$

These methods are all, of course, restricted to two-dimensional laminar flows of incompressible fluids at low dilation rates; i. e., areas of flow where the streamlines are parallel or nearly so. 
The most general method requires knowledge of the flow along at least one streamline. Numerical integration to reduce the velocity gradient to the desired fluid velocity is the rule.

Thus, it is seen that quantitative analysis of general flows typical of most engineering investigations is at the present time severly restricted. 


\section{REFERENCES}

1. J. H. Rushton. "Application of Fluid Mechanics and Similitude to Scale-Up Problems, ${ }^{\prime}$ Chem. Eng. Prog., Vol. 48, No. 1, pp 33-38. Vol. 48, No. 2, pp 95-102. 1952.

2. K. O. Kemp. "The Analysis of Laminar Flows from Streaming Birefringence Measurements," Civil Eng. and Public Works Review, Part 1, pp 767-770. June 1962; Part 2, pp 901-904. July 1962; Part 3, pp 1034-1035. August 1962.

3. M. Allen. "Centrifugation Can Select Solids by Density," Chem. Eng。, pp 182-183. December 15, 1958.

4. M. Allen. "Better Way to Trace Liquid Flow Patterns," Chem. Eng。, pp 148-150. May 4, 1959.

5. M. Allen and A. J. Yerman. "Neutral Density Beads for Flow Visualization, "Presentation Summaries, Symposium on Flow Visualization, Chap. 4. ASME Annual Meeting, New York, 1960.

6. E. F. Winter and J. H. Deterding. "Apparatus and Techniques for the Application of a Water Flow System to the Study of Aerodynamic Systems," Brit. J. Appl. Phys., Vol. 7, pp 247-259. 1956.

7. D. A. Van Meel and J. Vermig. "A Method for Flow Visualization and Measurement of Velocity Vectors in Three-Dimensional Flow Patterns in Water Models by Using Color Photography, "Appl. Sci. Res., Series A, Vol. 10, pp 109-117. 1960-61.

8. R. C. Binder. Advanced Fluid Dynamics and Fluid Machinery, Prentice-Hall, New York, 1951. pp 4.

9. S. Eskinazi. Principles of Fluid Mechanics, Allyn and Bacon, Inc. , Boston, 1962. pp 100-102, 113-117.

10. G. Birkhoff and E. H. Zarantonello. Jets, Wakes, and Cavities, Academic Press Inc., New York, 1957. pp 280-293.

11. O. J. Tietjens and L. Prandt1. Applied Hydro- and Aeromechanics, McGraw-Hill Book Co., Inc., New York, 1934. pp 40-41, 295.

12. L. Prandt1. Essentials of Fluid Dynamics, Hafner Publishing Co., New York, 1952. pp 117-135.

13. E. G. Coker and L. N. G. Filon. A Treatise on Photo-Elasticity, Cambridge University Press, 1931. pp 136.

14. J. W. Prados. The Analysis of Two-Dimensional Laminar Flow Utilizing a Doubly Refracting Liquid, The University of Tennessee, Available from University Microfilms, Ann Arbor, Michigan. 1957. (Ph. D. Thesis)

15. D。 R. Dewey. Visual Studies of Fluid Flow Patterns Resulting from Streaming Double Refraction, Massachusetts Institute of Technology, 1941. (Ph. D. Thesis) 
16. S. P. Sutera and H. Wayland. "Quantitative Analysis of TwoDimensional Flow By Means of Streaming Birefringence, " J. Appl. Phys., Vol. 32, No. 4, pp 721-730. 1961.

17. J. G. Knudsen and D. L. Katz. Fluid Dynamics and Heat Transfer, Engineering Research Institute, University of Michigan, Bulletin No. 37, pp 84-96. 1954. Also available from McGraw-Hill Book Co. , New York, pp 246-323, 1958.

18. H. Schlichting. Boundary Layer Theory, McGraw-Hill Book Co., New York, 1955. pp 131-138, 214-216.

19. V. L. Streeter. Fluid Dynamics, McGraw-Hill Book Co., New York, 1948. pp 256-257.

20. E. G. Richardson. Dynamics of Real Fluids, Edward Arnold and Co., London, 1950. pp 17-39.

21. V. L. Streeter. Fluid Dynamics, McGraw-Hill Book Co., New York, 1948. pp 174-177.

22. O. G. Tietjens and L. Prandtl. Applied Hydro-and Aerodynamics, McGraw Hill Book Co., Inc., New York, 1934. pp 36-38.

23. S. Eskinazi. Principles of Fluid Mechanics, Allyn and Bacon, Inc., Boston, 1952. pp 92.

24. F. N. Peebles, H. J. Garber, and S. H. Jury. "Preliminary Studies of Flow Phenomena Utilizing a Doubly Refractive Liquid," Proceedings - Third Midwestern Conference on Fluid Mechanics, pp 441-454, University of Minnesota Press, Minneapolis, 1953.

25. F. R. Hanna. "Streaklines in a Perturbed Shear Flow, "Physics of Fluids, Vol. 5, No. 6, pp 644-650. 1952.

26. S. J. Kline. "Opening Remarks, "Presentation Summaries, Symposium on Flow Visualization, Chap. 1. ASME Annual Meeting, New York, 1960.

27. W. Leaf. "Fluid Flow Study of Locomotive Firebox Design," Mech. Eng., Vol. 67, pp 586-590. September, 1945.

28. J. H. Chesters and A. R. Philip. "Flow Patterns in Ducts," J. Iron Steel Inst., Vol. 162, No. II, pp 385-391. 1949.

29. R. S. Howes and A. R. Philip. "Flow Visualization and Photography," J. Iron Steel Inst., Vol. 162, No. II, pp 392-401. 1949.

30. I. M. D. Halliday and A. R. Philip. "Flow Patterns ir Model Furnaces," J. Iron Steel Inst., Vol. 162, No. II, pp 401-415. 1949.

31. R. D. Collins and J. D. Tyler. "Experiments on Gaseous Mixing in Open-Hearth Furnace Models, "J. Iron Steel Inst. , Vol. 162, No. II, pp 457.1949. 
32. Fluid Models in Geophysics, Proceedings - First Symposium on Use of Models in Geophysical Fluid Dynamics. R. R. Long, Editor, Sponsored by U. S. Navy, Office of Navel Research in Cooperation with Geophysics Research Institute, and U. S. Weather Bureau, U. S. Government Printing Office, Washington D. C. , 1953.

33. C. C. Perry. "Visual Flow Analysis," Prod. Eng., pp 154-161. August, 1955.

34. A. W. Hendry. An Introduction to Photo-Elastic Analysis. Blackie and Son, Limited, London, 1948.

35. H. T. Jessop and F. C. Harris. Photoelasticity, Principles and Methods, Dover Publications, Inc., New York, 1950.

36. M. M. Frocht. Photoelasticity, J. Wiley, New York, 1941.

37. E. G. Coker and L. N. G. Filon. A Treatise on Photo-Elasticity, Cambridge University Press, London, 1931.

38. R. H. Humphrey. "Demonstration of Refraction Due to Motion, " Proc. Phys. Soc。, Vol. 35, pp 217-218。1923.

39. C. V. Ramon and K. S. Krishman. "A Theory of the Birefringence Induced by Flow in Liquids, "Phil. Mag., Vol. 5, pp 762-782. 1928.

40. E. D. Alcock and C. L. Sadron. "An Optical Method for Measuring the Distribution of Velocity Gradients in Two-Dimensional Flow, " Physics, Vol. 6, pp 92. 1935.

41. E. A. Hauser and D. R. Dewey. "Study of Liquid Flow, "Ind. Eng. Chem。, Vol. 31, pp 786 (Letter). 1939.

42. E。A.Hauser and D. R. Dewey. "Visual Studies of Flow Patterns," J. Phys。Chem., Vol. 46, pp 212. 1942 。

43. H. Wayland. "Streaming Birefringence as a Qualitative and Quantitative Flow Visualization Tool, "Presentation Summaries, Symposium on Flow Visualization, Chap. 9, ASME Annual Meeting, 1960.

44. A。 M. Binnie. "A Double-Refraction Method of Detecting Turbulence in Liquids, "Proc. Phys. Soc., Vol. 57, pp 390. 1954.

45. A. M. Binnie and J。S. Fowler. "A Study by a Double-Refraction Method of the Development of Turbulence in a Long Circular Tube, " Proc. Roy. Soc. A(London), 192, pp 32. 1947.

46. R. Weller. "The Optical Investigation of Fluid Flow, "J. Appl. Mechanics, Vol。14, pp A-103-A-107. 1947.

47. W. Philippoff. "Flow Birefringence and Stress, " J.Appl. Phys., Vol. 27, No. 9, pp 984。 1956.

48. W. Philippoff。 "Stress-Optical Analysis of Fluids, "Ind。 Eng。 Chem., Vol. 51, pp 883. 1959. 
49. H. G. Jerrard. "Turbulence in Apparatus for Measurement of Streaming Double Refraction," J. Appl. Phys., Vol. 21, pp 1007. 1950.

50. H. Wayland. "Streaming Birefringence as a Hydrodynamic Research Tool - Applied to a Rotating Cylinder Apparatus above the Transition Velocity," J. Appl. Phys., Vol. 26, pp 1197. 1955.

51. E. R. Lindgren. "Note on the Flow of Liquids in Tubes, "Appl. Sci. Research, A, Vol. 4, pp 313-316. 1954.

52. J. W. Prados and F. N. Pebbles. "Two-Dimensional Laminar-Flow Analysis, Utilizing a Doubly Refractive Liquid, "A.I.Ch. E. Journal, Vol. 5, No. 2, pp 225-234. 1959.

53. B. Rosenberg. "The Motion of Small. Slender Particles in Viscouse Incompressible Flow, "Proceedings 1st U. S。 Nat'1 Congress on Applied Mechanics, pp 807-811. 1951.

54. H. Wayland. "Streaming Birefringence of Rigid Macromolecules in General Two-Dimensional Laminar Flow, "J. Chem. Phys., Vol. 33, No. 3, pp. 769. 1960.

55. H. G. Jerrard. "Theories of Streaming Double Refraction, "Chem. Rev., Vol. 59, pp 345-427. 1959.

56. K. O. Kemp. Properties of Bentonite Suspensions and Their Use in the Study of Engineering Problems of Laminar Flow, University College, Longdon, England، 1961. (Ph. D. Thesis)

57. W. R. Schowalter. "Rheological Equations of State, "Chem. Eng. Prog., Vol. 60, No. 8, pp 52-56. August, 1964. 


\section{INTERNAL DISTRIBUTION}

\section{Copy Number}

$$
\begin{aligned}
1 & \text { F. W. Albaugh } \\
2 & \text { J. A. Ayres } \\
3 & \text { J. M. Batch } \\
4 & \text { J. C. Fox } \\
5 & \text { H. Harty } \\
6 & \text { D. P. Schively } \\
7-20 & \text { D. H. White } \\
21 & \text { F. R. Zaloudek } \\
22-26 & \text { 300 Area File } \\
27 & \text { Technical Publications }
\end{aligned}
$$

\section{EXTERNAL DISTRIBUTION (Special)}

\section{Number of Copies}

1 Atomic Energy Commission, Washington Military Liaison Committee

Attn: Captain D. E. McCoy, Staff Assistant to the Chairman

General Electric Company, Richland

Attn:

D. L. Condotta (1)

L. P. Reinig (1)

R. H. Shoemaker (1) 


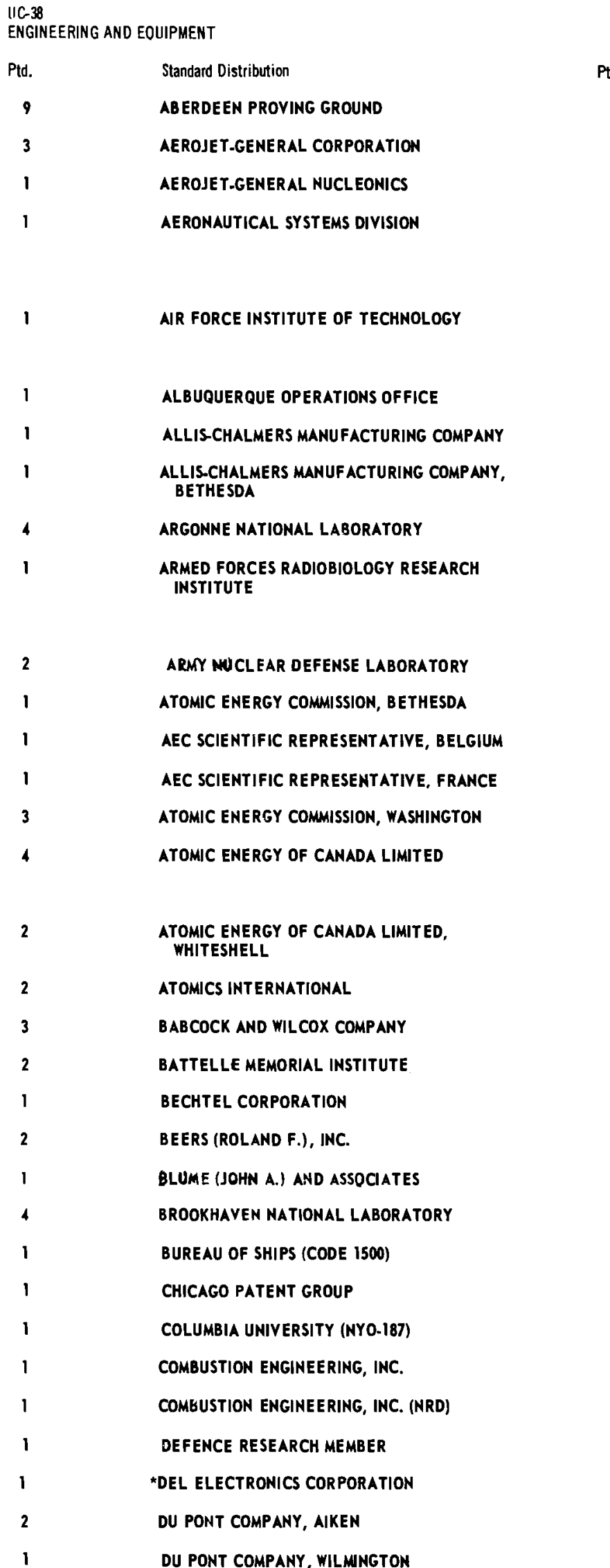

TID-4500

(37th Ed.)

Ptd.

1

1

2

1

1

1

1

1

2

1

1

2

1

1

l

1

1

1 1

1

2

1

1
Standard Distribution

FUNDAMENTAL METHODS ASSOCIATION

GENERAL ATOMIC DIVISION

GENERAL ELECTRIC COMPANY, CINCINMATI

GENERAL ELECTRIC COMPANY, RICHLAND

GENERAL ELECTRIC COMPANY, SAN JOSE

GENERAL NUCLEAR ENGINEERING CORPORATION

GOODYEAR ATOMIC CORPORATION

HOMESTAKE MINING COMPANY

IOWA STATE UNIVERSITY

KNOLLS ATOMIC POWER LABORATORY

LOCKHEE D-GEORGIA COMPANY

LOCKHEED MISSILES AND SPACE COMPANY (NASA)

LOS ALAMOS SCIENTIFIC LABORATORY

LOVELACE FOUNDATION FOR MEDICAL EDUCATION AND RESEARCH

MARE ISLAND NAVAL SHIPYARD

MARTIN-MARIE TTA CORPORATION

MOUND LABORATORY

MUESER, RUTLEDGE, WENTWORTH AND JOHNSTON

MASA SCIENTIFIC AND TECHNICAL INFORMATION FACILITY

NATIONAL LEAD COMPANY OF OHIO

NEVADA OPERATIONS OFFICE

NEW YORK UNIYERSITY (BEDERSON)

NRA, INC.

NUCLEAR TECHNOLOGY CORPORATION

OFFICE OF ASSISTANT GENERAL COUNSEL FOR PATENTS (AEC)

OFFICE OF THE CHIEF OF ENGINEERS

OHIO STATE UNIVERSITY

PETROLEUM CONSULTANTS

PHILLIPS PETROLEUM COMPANY (NRTS)

PHYSICS INTERNATIONAL, INC.

POWER REACTOR DEVELOPMENT COMPANY

PRATT AND WHITNEY AIRCRAFT DIVISION 
Pid.

1
Standard Distribution

PURDUE UNIVERSITY

RUTGERS UNIVERSITY (LIBRARY)

SANDIA COR PORATION, ALBUQUERQUE

SANDIA CORPORATION, LIVERMORE

SOUTHWEST RESEARCH INSTITUTE

STANFORD UNIVERSITY (SLAC)

SYLYANIA ELECTRIC PRODUCTS, INC.

TENNESSEE VALLEY AUTHORITY

TRW SPACE TECHNOLOGY LABORATORIES (NASA)

UNION CARBIDE CORPORATION (ORGDP)

UNION CARBIDE CORPORATION (ORNL)

ENGINEERING AND EQUIPMENT

Ptd.

325

75
Standard Distribution

UNITED NUCLEAR CORPORATION (NDA)

UNIYERSITY OF CALIFORNIA, BERKELEY

UNIYERSITY OF CALIFORNIA, LIVERMORE

UNIVERSITY OF PUERTO RICO

WESTINGHOUSE BETTIS ATOMIC POWER LABORATORY

WESTINGHOUSE ELECTRIC CORPORATION

WESTINGHOUSE ELECTRIC CORPORATION (NASA)

WHITE SANDS MISSILE RANGE

DIVISION OF TECHNICAL INFORMATION EXTENSION

CLEARINGHOUSE FOR FEDERAL SCIENTIFIC AND TECHNICAL INFORMATION 